\title{
Scale-dependent Response of Fluid Turbulence under Variation of the Large-scale Forcing
}

\author{
Dissertation \\ for the award of the degree \\ Doctor rerum naturalium \\ of the Georg-August-Universität Göttingen \\ within the doctoral program \\ Physics of Biological and Complex Systems \\ of the Georg-August University School of Science (GAUSS)
}

submitted by

Fabio Di Lorenzo

from Völklingen

Göttingen, 2014 


\section{Thesis Committee:}

Prof. Dr. Eberhard Bodenschatz

Laboratory for Fluid Dynamics, Pattern Formation, and Biocomplexity, Max Planck

Institute for Dynamics and Self-Organization

Prof. Dr. Jörg Enderlein

III. Physical Institute Biophysics / Complex Systems, Georg-August-Universität Göttingen

Prof. Dr. Björn Hof

Nonlinear Dynamics and Turbulence, Institute of Science and Technology Austria

\section{Members of the Examination Board:}

\section{Referee: Prof. Dr. Eberhard Bodenschatz}

Laboratory for Fluid Dynamics, Pattern Formation, and Biocomplexity, Max Planck

Institute for Dynamics and Self-Organization

2nd Referee: Prof. Dr. Jörg Enderlein

III. Physical Institute Biophysics / Complex Systems, Georg-August-Universität Göttingen

\section{Further Members of the Examination Board:}

Prof. Dr. Björn Hof

Nonlinear Dynamics and Turbulence, Institute of Science and Technology Austria

Dr. Haitao Xu

Laboratory for Fluid Dynamics, Pattern Formation, and Biocomplexity, Max Planck

Institute for Dynamics and Self-Organization

Dr. Olga Shishkina

Laboratory for Fluid Dynamics, Pattern Formation, and Biocomplexity, Max Planck

Institute for Dynamics and Self-Organization

Prof. Dr. Marcus Müller

Institute for Theoretical Physics, Georg-August-Universität Göttingen

Date of oral examination: February 3, 2015 


\section{Contents}

\begin{tabular}{lll}
\hline & Introduction & 1
\end{tabular}

\begin{tabular}{|llr}
\hline 2 & Theoretical Background & 8
\end{tabular}

2.1 Navier-Stokes Equations . . . . . . . . . . . . . . . . . . . . 8

2.2 The Theory of Kolmogorov (1941) . . . . . . . . . . . . . . . . . . . 11

2.3 Second-Order Velocity Structure Function . . . . . . . . . . . . . . 17

2.4 Kolmogorov Equation. . . . . . . . . . . . . . . . . . . . . . 20

2.5 Perturbing a Turbulent Flow . . . . . . . . . . . . . . . . . . . . . 23

\begin{tabular}{lll}
\hline 3 & Experimental Setup & 27
\end{tabular}

$3.1 \quad$ Lagrangian Exploration Module . . . . . . . . . . . . . . . . . . . . 28

3.2 Lagrangian Particle Tracking . . . . . . . . . . . . . . . . . . . . 34

3.2.1 Camera Setup and Calibration . . . . . . . . . . . . . . . 35

3.2 .2 Particle Finding . . . . . . . . . . . . . . . . . . . . . . . . . 38

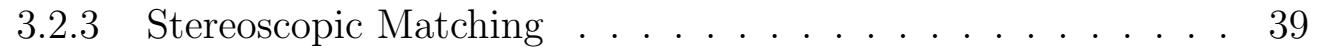


3.2 .4 Particle Tracking . . . . . . . . . . . . . . . . . . . 42

3.3 Real Time Image Compression System . . . . . . . . . . . . . . . . 45

3.3.1 High-speed Cameras . . . . . . . . . . . . . . . 50

$3.3 .2 \quad$ FPGA System . . . . . . . . . . . . . . . . . 53

3.4 Nd:YAG Laser . . . . . . . . . . . . . . . . . . . . . . . 56

3.5 Automation of the Experimental Setup . . . . . . . . . . . . . . . . 57

$\begin{array}{lll}4 & \text { Results } & 66\end{array}$

4.1 Steady State . . . . . . . . . . . . . . . . . . . . . . . 68

4.2 Step-up of Energy Injection . . . . . . . . . . . . . . . . . . . 76

4.3 Decay of Turbulence . . . . . . . . . . . . . . . . . . . 90

5 Summary and Outlook 103

\begin{tabular}{ll}
\hline Bibliography & 107
\end{tabular}

\begin{tabular}{|ll}
\hline Acknowledgments & 117
\end{tabular}

\begin{tabular}{ll}
\hline Curriculum Vitae & 119
\end{tabular} 


\section{Chapter 1}

\section{Introduction}

In the flow of a fluid, two extreme states can be distinguished, i.e. laminar and turbulent. A flow is called laminar if thin layers of the fluid move side by side, otherwise the flow is called turbulent. While a laminar flow is highly ordered and small perturbations are damped, a turbulent flow is characterized by strong fluctuations of the velocity field in time and space leading to irregular and chaotic flow patterns. Figure 1.1 shows laminar smoke rising from an incense stick and developing more and more turbulent structures.

The first systematic investigation concerning the transition from laminar to turbulent flow was performed by Osbourne Reynolds (1883). It is in his honor that we call the dimensionless parameter determining whether a flow is turbulent or not the Reynolds number. The Reynolds number of a flow is defined as $R e=\frac{U L}{\nu}$, where 


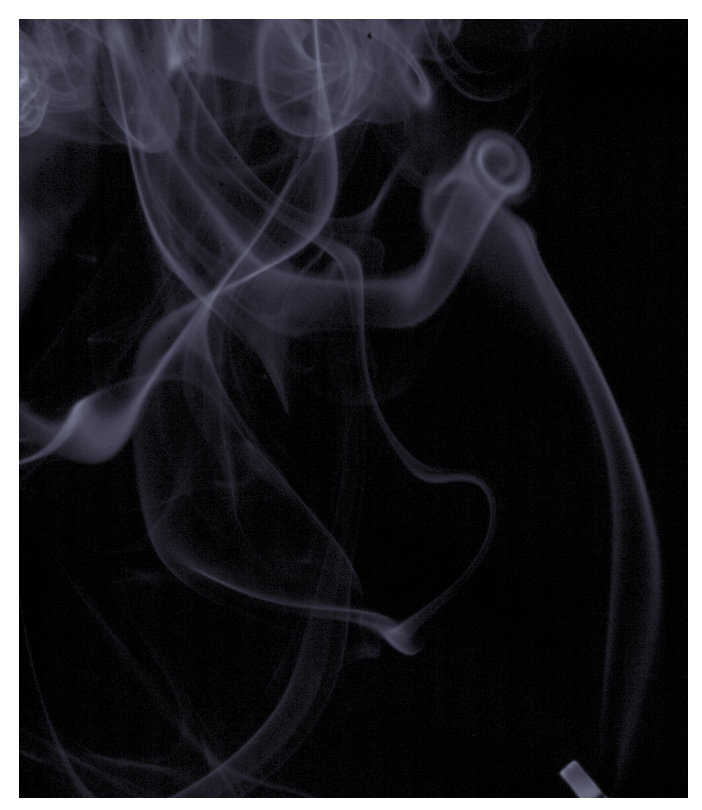

Figure 1.1: Smoke rising from an incense stick in the lower right corner. It is laminar at first and then becomes turbulent as it rises.

$U$ is a characteristic velocity, $L$ is a characteristic length and $\nu$ is the kinematic viscosity of the fluid. One expects flows in similar geometries to behave similarly if they have the same Reynolds number. Note that, usually there are more than one characteristic velocity and length scales available for defining the Reynolds number. The same definition should be used when comparing flows in different observations. For pipe flows e.g. it is common to choose $U$ to be the mean flow velocity and $L$ to be the pipe diameter. A different choice, say, the center line velocity for $U$ and the radius of the pipe for $L$, would merely change the numeric 
values of the Reynolds number. As a consequence, the Reynolds number that marks the transition from a laminar to a turbulent state of the flow depends on the flow geometry and the choice of $U$ and $L$. In the above example of a pipe flow, the transition occurs at about $R e \sim 2040$ (Avila et al. 2011), while a flow between parallel plates becomes turbulent around $R e \sim 1100$ 1 (Hinze, 1975).

The Reynolds number can be interpreted as the ratio of inertial forces to viscous forces. For small Re, the viscous forces dominate and have a strong smoothing effect on the flow. As a consequence, the flow field is insensitive to small perturbations and the flow stays laminar. On the contrary, high Re flows are dominated by inertial forces and are turbulent since the viscous forces cannot sufficiently damp the strong fluctuations occurring in the velocity field. There may also exist a range of $R e$ where perturbations can trigger the transition from a laminar to a turbulent flow, but turbulence does not necessarily persist.

In nature we find turbulence, e.g., in astronomic flows where it is involved in the formation of stars and planets. The earth's climate is governed by turbulent atmospheric and oceanic flows. Even the pulsating flow in the cardiovascular system is turbulent. In our daily life, turbulence is omnipresent because viscosities of the involved fluids in natural and technical flows are usually so small that high Reynolds numbers are easily reached. Considering the flow of water

\footnotetext{
${ }^{1}$ In this case $R e$ is defined with $U$ being the mean flow velocity and $L$ being the distance between the plates.
} 
$\left(\nu \sim 10^{-6} \mathrm{~m}^{2} / \mathrm{s}\right.$ at $\left.20^{\circ} \mathrm{C}\right)$ through a pipe with a diameter of $2 \mathrm{~cm}$, a Reynolds number of $R e=2040$ is reached with a flow velocity of only $10.2 \mathrm{~cm} / \mathrm{s}$. This example shows that turbulence occurs in nearly every fluid flow one usually encounters. Compared to laminar flows, turbulence enhances mixing and increases the rate of transport of matter, momentum and heat. Therefore, turbulence also plays an important role in industrial flows. In combustion engines, e.g., oxygen and fuel need to be mixed efficiently in very short times. In this case we take advantage of turbulence. But in other applications turbulence is unfavorable, when pumping oil through pipelines, e.g., turbulence reduces the efficiency of the process due to an increased pressure drop.

Now let us consider a simple example, and try to get some deeper insights from it. If we stir the coffee in a cup we produce a turbulent flow. One could also say, as we pass kinetic energy from the spoon on to the coffee, we inject energy in the flow. If we stop stirring, the flow comes to rest after a while. This shows that fluid flows are dissipative and turbulence needs energy to be injected in order to persist. One also notices that the turbulent small-scale motions in the coffee cup die out fairly quickly if we stop stirring, while the remaining large-scale circulation dies out later. This shows that dissipation acts stronger on the small scale motions as they are attenuated faster. 
The example discussed above reveals an important question that arises when dealing with turbulent flows, that is, how the energy injected in the flow is transported from large to small scales where it is dissipated. The injection of energy is performed on large length scales and the size of this energy injection scale depends on the geometrical properties of the flow. It could be the size of an object disturbing the flow or the size of a propeller or a spoon stirring the fluid etc. The dissipation of the injected energy, however, takes place on the smallest scales of the flow where viscous dissipation transforms kinetic energy into heat. In order to describe what happens in between, Richardson (1922) introduced the idea of the energy cascade. In this picture a turbulent flow consists of a superposition of eddies of different sizes. Regarding the word eddy Batchelor (1950) wrote that

"... the word 'eddy' does not refer to any particular local distribution of velocity. It is merely a concise term for a component of motion with a certain length scale, i.e. an arbitrary flow pattern characterized by size alone."

The kinetic energy contained in eddies of large size is transferred to smaller eddies, which themselves pass the energy on to even smaller eddies and so on. Following this idea, energy cascades from the largest scales of a flow where it is injected, down to the smallest scales where viscosity dominates the behavior of the fluid motion and dissipates the energy into heat. 
In 1941, Kolmogorov derived a statistical theory based on the Navier-Stokes equations, the equations of motion of a fluid. With the energy cascade picture in mind he provided predictions for statistical quantities of homogeneous turbulent flows at high Reynolds numbers. Kolmogorov's theory, referred to as K41, states that the average rate of energy injection on the large scales equals the average rate of energy dissipation on the small scales. Moreover, for high Reynolds number flows there exists a range in which statistical quantities, like velocity correlations etc., solely depend on the rate of energy transfer and the scale at hand.

Most efforts in turbulence research, be it experimentally, numerically or theoretically, focus on statistically stationary turbulence, i.e., a turbulent flow driven by a constant energy input. Statistical properties of such flows are independent of time and can be compared to predictions given by K41. On the other hand, deeper insight on the energy cascade process might be gained, by perturbing the energy input and measure the response at different scales of the flow. We therefore set up an experiment to measure the temporal evolution of energy transfer at different scales in a turbulent flow that was subject to a perturbation of the energy injection. Two types of experiments were performed. In the first type a step-function-like increase of the energy input was used to force the turbulent flow. In the second case the forcing of a fully developed turbulent flow was switched off to observe the decay of the turbulence. 
Experiments investigating the scale dependent response of a turbulent flow to a perturbation of the large-scale forcing have, to the best knowledge of the author, not been done so far. This is due to the complexity of the endeavor and the limitations of state-of-the-art measurement techniques. The approach to overcome these limitations and the hardware developed for this purpose is also documented in this thesis.

In Chapter 2 the theoretical framework of turbulence needed for this thesis is introduced and relevant previous work is reviewed. The experimental setup and the measurement technique is described in Chapter 3. The results of the conducted experiments are presented in Chapter 4. Finally, the summary and an outlook is given in Chapter 5 . 


\section{Chapter 2}

\section{Theoretical Background}

\subsection{Navier-Stokes Equations}

When considering the balance of forces acting on a fluid element one can derive the equations of motion for a fluid (Davidson, 2004), i.e., the Navier-Stokes equations

$$
\frac{\partial \boldsymbol{u}(\boldsymbol{x}, t)}{\partial t}+(\boldsymbol{u}(\boldsymbol{x}, t) \cdot \nabla) \boldsymbol{u}(\boldsymbol{x}, t)=-\frac{1}{\rho} \nabla P(\boldsymbol{x}, t)+\nu \nabla^{2} \boldsymbol{u}(\boldsymbol{x}, t),
$$

where $\boldsymbol{u}(\boldsymbol{x}, t)$ denotes the velocity field, $P(\boldsymbol{x}, t)$ represents the pressure field and $\rho$ and $\nu$ are the density and the kinematic viscosity of the fluid, respectively. Throughout this thesis, we only consider the case of constant fluid density and constant fluid viscosity. The Navier-Stokes equations, Eq. (2.1), together with the 
continuity equation that arises from conservation of mass for constant density, $\nabla$. $\boldsymbol{u}(\boldsymbol{x}, t)=0$, are believed to fully describe the behavior of incompressible Newtonian fluids.

The meaning of Eq. (2.1) can be better appreciated after non-dimensionalizing the equation. This is done by using a characteristic length scale $L$ and a characteristic velocity $U$ to non-dimensionalize each variable:

$$
\tilde{\boldsymbol{x}}=\frac{\boldsymbol{x}}{L} \quad \tilde{t}=\frac{t U}{L} \quad \tilde{\boldsymbol{u}}=\frac{\boldsymbol{u}}{U} \quad \tilde{P}=\frac{P}{\rho U^{2}} .
$$

Replacing all variables in Eq. (2.1) according to Eq. (2.2) and dividing by $U^{2} / L$ leads to the Navier-Stokes equations in dimensionless form

$$
\frac{\partial \boldsymbol{u}(\boldsymbol{x}, t)}{\partial t}+(\boldsymbol{u}(\boldsymbol{x}, t) \cdot \nabla) \boldsymbol{u}(\boldsymbol{x}, t)=-\nabla P(\boldsymbol{x}, t)+\frac{1}{R e} \nabla^{2} \boldsymbol{u}(\boldsymbol{x}, t),
$$

where $R e=U L / \nu$ is the Reynolds number. In order to increase the readability of Eq. (2.3), the tildes were dropped. It can be seen that the Reynolds number appears naturally as the only control parameter when non-dimensionalizing the equations of motion of a fluid.

While the first term on the left hand side of Eq. (2.3) represents the temporal change of the velocity field, the second term corresponds to the momentum transport by fluid motion and is therefore called convective term. The second term 
on the right hand side is responsible for viscous dissipation. It is easy to see the role of the Reynolds number on the behavior of the fluid in this form of the equations of motion. While the convective term is responsible for the chaotic nature of fluid flows as it enhances perturbations, the viscous term is able to damp out these disturbances in low Reynolds number flows and the flow stays laminar. In high Reynolds number cases the viscous term becomes negligible and turbulence develops.

The first term on the right hand side, the pressure term, can be related to the velocity field. When taking the divergence of Eq. (2.3) and using the continuity condition one finds that the pressure field has to satisfy Poisson's equation

$$
\nabla^{2} P(\boldsymbol{x}, t)=-\nabla \cdot[(\boldsymbol{u}(\boldsymbol{x}, t) \cdot \nabla) \boldsymbol{u}(\boldsymbol{x}, t)]
$$

It is known form electrodynamics Nolting, 2007) that the solution to Eq. 2.4 has the form

$$
P(\boldsymbol{x}, t)=\frac{1}{4 \pi} \iiint \frac{\nabla \cdot\left[\left(\boldsymbol{u}\left(\boldsymbol{x}^{\prime}, t\right) \cdot \nabla\right) \boldsymbol{u}\left(\boldsymbol{x}^{\prime}, t\right)\right]}{\left|\boldsymbol{x}-\boldsymbol{x}^{\prime}\right|} d \boldsymbol{x}^{\prime}
$$

This shows that Eq. 2.3 is a deterministic equation with three unknowns, the components of the velocity field, to solve for. Nevertheless, it is very difficult to solve the Navier-Stokes equations analytically, except for some special cases 
(Batchelor, 1967). In addition to that, solving the problem numerically is computationally very expensive $($ Pope, 2000$)$ due to the vast number of degrees of freedom and the non-local character of the equations, which can be seen in Eq. 2.5). In order to reduce the number of degrees of freedom and to get to a description that is easier to relate to measurable quantities, it is desirable to adapt a statistical description of turbulence.

\subsection{The Theory of Kolmogorov (1941)}

Before we move to the statistical description of turbulence, let us have a closer look at the phenomenon of the energy cascade. The energy cascade, as introduced by Richardson (1922), describes the process by which energy is transferred in a turbulent flow - from the largest scales, where the energy is injected, to the smallest scales, where dissipation occurs. According to this idea, one considers a turbulent flow to consist of eddies of different sizes. Large eddies will break up into smaller eddies and pass on their energy to them. These smaller eddies will also break up and pass on their energy to eddies that are even smaller. This process goes on until the energy has reached the smallest scales of the flow where viscosity dominates and the energy is converted into heat. 
Kolmogorov (1941b) extended the idea of the energy cascade further, in order to make predictions for statistical quantities in turbulent flows. He states that eddies of size $l$ have a characteristic velocity $u(l)$ and therefore could be assigned a scale-dependent Reynolds number $R e(l)=\frac{u(l) l}{\nu}$. Since the Reynolds number represents the ratio of inertial to viscous forces, Kolmogorov argued, that the smallest scales of a turbulent flow where the viscous forces dominate and energy is dissipated have a Reynolds number of unity. The length scale at which this occurs is called the Kolmogorov length scale, and is usually denoted $\eta$.

Furthermore, for large Re Kolmogorov made assumptions about the conditions in different scale ranges and proposed three subranges (Fig. 2.1). On the largest scales of a flow, $L$, energy is injected, say by a fan with a diameter of $L$ or by a grid with the mesh size $L$. Scales where $l \sim L$ are called energy containing range. The behavior of the fluid in the energy containing range is dominated by the way energy is injected and by the boundaries of the flow. This means that statistics of the large scales in, e.g., grid turbulence in a wind tunnel or in a von Kármán flow (flow between two counter-rotating propellers in a cylindric vessel) can be very different, because the geometries of the apparatuses and the means of forcing are different.

However, for scales that are much smaller than the energy containing range $(l \ll L)$, it appears that, the statistics of a turbulent flow are independent of the 


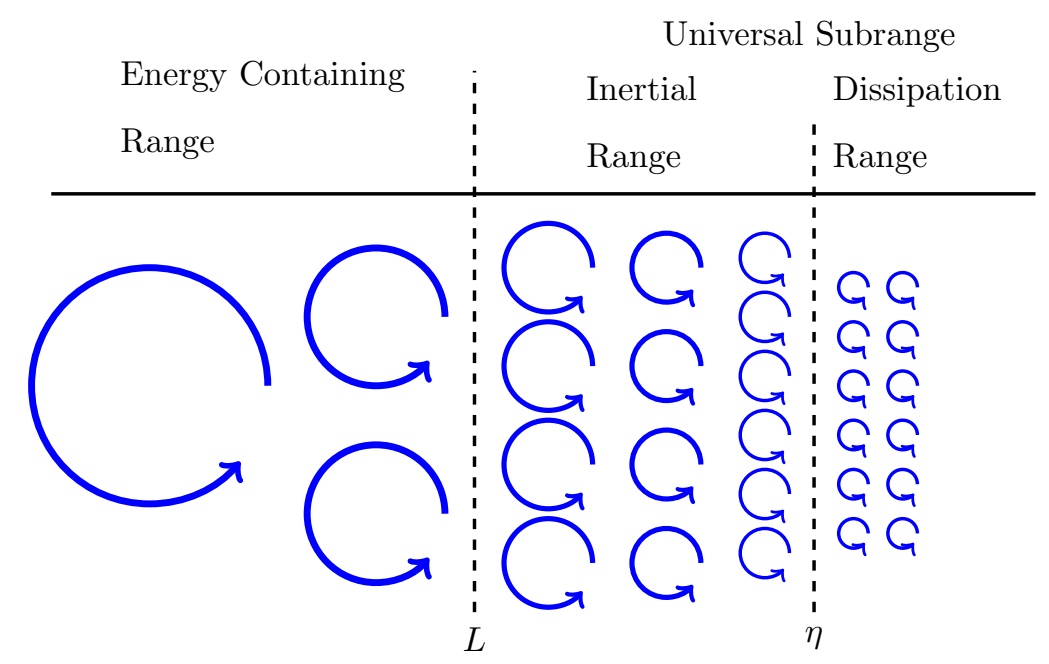

Figure 2.1: A sketch of the energy cascade and the three regimes proposed by Kolmogorov. Energy is injected in the flow at scales on the order of $L$, the energy containing range. Then the eddies break up into smaller and smaller eddies and pass on their energy down the cascade until the energy is dissipated. The scale range smaller than $L$ is called universal subrange and is divided into two ranges. The inertial range $(\eta \ll l \ll L)$ where statistics solely depend on the dissipation rate $\epsilon$ and the dissipation range $(l \lesssim \eta)$ where the statistics depend on $\epsilon$ and $\nu$.

way in which it was produced and are universal for all turbulent flows Saddoughi \& Veeravalli, 1994). This range is called universal subrange and can be further divided into two subranges. The very smallest scales $(l \lesssim \eta)$, where molecular interactions dominate and the energy is dissipated, are called dissipation range. In between the energy containing and the dissipation ranges is a range of scales 
$(\eta \ll l \ll L)$ where the statistics are purely dominated by inertia, the inertial range. This range only exists for very high Reynolds number.

One of the most important assumptions of K41 is that, due to energy conservation, the energy transfer through the scales from the energy containing range all the way down to the dissipation range stays constant for statistically stationary turbulence. Therefore the energy dissipation rate per unit mass equals the energy transfer rate per unit mass and is called $\epsilon$. To summarize the discussion above, Kolmogorov formulated three hypotheses for homogeneous turbulence at high Reynolds numbers (see also Frisch (1995); Pope (2000); Davidson (2004)).

Hypothesis of local isotropy: In high Reynolds number flows the statistics in the universal subrange are isotropic. That means, no matter how the flow is created, the information of the large scale structure is lost. Therefore the small scale statistics of all high Reynolds number flows are similar and can be compared.

First similarity hypothesis: In the dissipation range of high Reynolds number flows, statistics are universal and only depend on $\nu, \epsilon$ and the scale itself.

Second similarity hypothesis: In the inertial range of high Reynolds number flows, statistics are universal and only depend on $\epsilon$ and the scale itself. 
With the introduction of his three hypotheses, Kolmogorov provided a useful framework to predict the behavior of statistical quantities in the universal subrange of turbulent flows of high Reynolds number. Additionally, the assumption of universality implies that small scale statistics of different turbulent flows are the same when normalized by appropriate parameters. Furthermore, since the statistics in the universal subrange are solely depending on $\epsilon$ and $\nu$, the parameters suitable for normalization must be fully characterized by these two quantities. Based on this assumption and dimensional reasoning the Kolmogorov scales for length $\eta$, velocity $u_{\eta}$ and time $\tau_{\eta}$ are defined as

$$
\begin{aligned}
\eta & =\left(\frac{\nu^{3}}{\epsilon}\right)^{1 / 4} \\
u_{\eta} & =(\nu \epsilon)^{1 / 4} \\
\tau_{\eta} & =\left(\frac{\nu}{\epsilon}\right)^{1 / 2} .
\end{aligned}
$$

When calculating the Reynolds number using the Kolmogorov scales we find that $\operatorname{Re}(\eta)=\frac{u_{\eta} \eta}{\nu}=1$, which is consistent with the assumption that viscous and inertial forces become comparable at the Kolmogorov scale $\eta$.

Following the argumentation of Kolmogorov that the energy transfer rate $\epsilon$ is constant throughout the scales and that statistical quantities in the inertial range only depend on $\epsilon$ and the scale itself, dimensional analysis shows that the 
characteristic velocity $u(l)$ for an eddy of size $l$ in the inertial range has to satisfy

$$
u(l) \sim(\epsilon l)^{1 / 3}
$$

Using Eq. 2.7 and Eq. 2.6, the scaling of the Reynolds number, $R e(l)=\frac{u(l) l}{\nu}$, can be obtained (Pope, 2000).

$$
\begin{aligned}
\operatorname{Re}(l)^{3 / 4} & \sim \frac{l}{\eta} \\
\operatorname{Re}(l)^{1 / 4} & \sim \frac{u(l)}{u_{\eta}} \\
\operatorname{Re}(l)^{1 / 2} & \sim \frac{\tau(l)}{\tau_{\eta}}
\end{aligned}
$$

This shows that, as $l$ increases the Reynolds number also increases. Moreover, the characteristic velocities $u(l)$ and the so called eddy turn-over times $\tau(l)$ increase accordingly. In addition, Eq. (2.8) shows that high Reynolds number flows have a larger scale separation than low Reynolds number flows.

In the following the velocity structure functions are introduced and it is shown, how K41 can be applied to predict the properties of the velocity structure functions, in particular for those of second order. 


\subsection{Second-Order Velocity Structure Function}

One of the simplest statistical quantities that contains information related to a certain scale of a turbulent flow is the second order velocity structure function tensor

$$
D_{i j}(\boldsymbol{r}, t)=\left\langle\left[u_{i}(\boldsymbol{x}+\boldsymbol{r}, t)-u_{i}(\boldsymbol{x}, t)\right]\left[u_{j}(\boldsymbol{x}+\boldsymbol{r}, t)-u_{j}(\boldsymbol{x}, t)\right]\right\rangle .
$$

It contains covariances of the components of velocity differences between two points $\boldsymbol{x}$ and $\boldsymbol{x}+\boldsymbol{r}$. Since we focus on homogeneous turbulence, $D_{i j}(\boldsymbol{r}, t)$ does not depend on $\boldsymbol{x}$. The $\langle\ldots\rangle$ in Eq. 2.9 denotes the ensemble average and is the same as the spatial average in homogeneous turbulence. In isotropic turbulence, the off diagonal elements of $D_{i j}(\boldsymbol{r}, t)$ vanish and the tensor can be fully described by $D_{L L}(r, t)$ and $D_{N N}(r, t)$, the longitudinal and transversal second-order velocity structure functions, respectively. While the longitudinal structure function,

$$
D_{L L}(r, t)=\left\langle\left[u_{\|}(\boldsymbol{x}+\boldsymbol{r}, t)-u_{\|}(\boldsymbol{x}, t)\right]^{2}\right\rangle,
$$

represents the component of the velocity difference along the separation vector $\boldsymbol{r}$, the transversal structure function,

$$
D_{N N}(r, t)=\left\langle\left[u_{\perp}(\boldsymbol{x}+\boldsymbol{r}, t)-u_{\perp}(\boldsymbol{x}, t)\right]^{2}\right\rangle,
$$


stands for the component perpendicular to $\boldsymbol{r}$ (Fig. 2.2). In isotropic turbulence, both $D_{L L}(r, t)$ and $D_{N N}(r, t)$ are independent of the direction of $\boldsymbol{r}$.

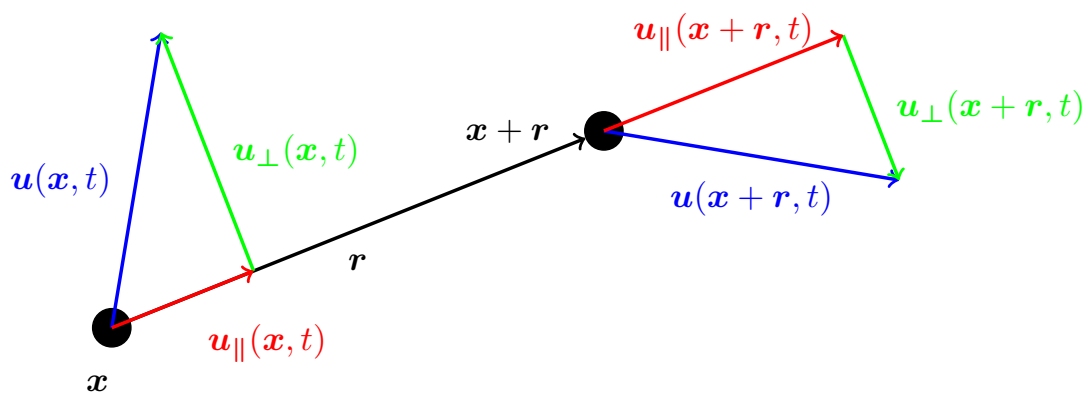

Figure 2.2: Two fluid particles at the positions $\boldsymbol{x}$ and $\boldsymbol{x}+\boldsymbol{r}$ are shown with the separation vector $\boldsymbol{r}$. The blue vectors show the velocities of the particles. The red vectors are the projections of the velocities onto the separation vector and are used to calculate $D_{L L}(r, t)$. The green vectors are the velocity components perpendicular to the separation vector and are used to calculate $D_{N N}(r, t)$.

In this case, $D_{i j}(r, t)$ can be fully expressed by $D_{L L}(r, t), D_{N N}(r, t)$ and the isotropic second order tensors $\delta_{i j}$ (the Kronecker symbol) and $r_{i} r_{j}$ (Pope, 2000):

$$
D_{i j}(r, t)=D_{N N}(r, t) \delta_{i j}+\left[D_{L L}(r, t)-D_{N N}(r, t)\right] \frac{r_{i} r_{j}}{r^{2}}
$$

Taking the derivative of Eq. 2.9 with respect to $r_{i}$ yields $\frac{\partial}{\partial r_{i}} D_{i j}(r, t)=0$ due to the incompressibility condition. Using this result with Eq. (2.12), a relation 
between $D_{L L}(r, t)$ and $D_{N N}(r, t)$ can be derived:

$$
\begin{aligned}
D_{N N}(r, t) & =D_{L L}(r, t)+\frac{r}{2} \frac{\partial}{\partial r} D_{L L}(r, t) \\
& =\frac{1}{2 r} \frac{\partial}{\partial r}\left(r^{2} D_{L L}(r, t)\right) .
\end{aligned}
$$

That means, in homogeneous isotropic turbulence the full tensor $D_{i j}(r, t)$ is given by one measurable scalar function $D_{L L}(r, t)$ or $D_{N N}(r, t)(\overline{\text { Pope }}, 2000)$.

A prediction that can be made from $\mathrm{K} 41$ for the behavior of $D_{L L}(r, t)$ in the inertial range, is based on the second similarity hypothesis. Since it states that statistics here only depend on $\epsilon$ and $r$, by dimensional analysis one finds that

$$
D_{L L}(r, t)=C_{2}(\epsilon r)^{2 / 3},
$$

where $C_{2}$ is called the Kolmogorov constant. The value of $C_{2}$ has been determined experimentally to be $\sim 2$ (Pope, 2000; Sreenivasan, 1995). Combining Eq. (2.13) and Eq. (2.14), a similar expression for $D_{N N}(r, t)$ can be found

$$
D_{N N}(r, t)=\frac{4}{3} C_{2}(\epsilon r)^{2 / 3} .
$$




\subsection{Kolmogorov Equation}

The Kolmogorov equation (Kolmogorov, 1941a) is an exact formula for time dependent statistics of the velocity structure functions. It is based on the Navier-Stokes equations, which in component notation read

$$
\frac{\partial u_{j}(\boldsymbol{x}, t)}{\partial t}=-u_{i}(\boldsymbol{x}, t) \frac{\partial u_{j}(\boldsymbol{x}, t)}{\partial x_{i}}-\frac{1}{\rho} \frac{\partial P(\boldsymbol{x}, t)}{\partial x_{j}}+\nu \frac{\partial^{2} u_{j}(\boldsymbol{x}, t)}{\partial x_{i} \partial x_{i}}
$$

In the following, the principle steps of its derivation are outlined. We start with the time derivative of Eq. 2.9

$$
\frac{\partial D_{i j}(r, t)}{\partial t}=\left\langle v_{i} \frac{\partial v_{j}}{\partial t}\right\rangle+\left\langle v_{j} \frac{\partial v_{i}}{\partial t}\right\rangle
$$

where $v_{k}=u_{k}(\boldsymbol{x}+\boldsymbol{r}, t)-u_{k}(\boldsymbol{x}, t)$ is the velocity difference over a distance $r$. The

time derivatives $\frac{\partial v_{k}}{\partial t}$ can then be replaced with help of the Navier-Stokes equations, Eq. (2.16), yielding

$$
\frac{\partial D_{i j}(r, t)}{\partial t}=-\frac{\partial D_{i j k}(r, t)}{\partial r_{k}}+2 \nu \frac{\partial^{2} D_{i j}(r, t)}{\partial r_{k} \partial r_{k}}-4 \nu\left\langle\frac{\partial v_{i}}{\partial r_{k}} \frac{\partial v_{j}}{\partial r_{k}}\right\rangle
$$

Here, the pressure gradient terms dropped out due to isotropy von Kármán \& Howarth, 1938; Hinze, 1975). $D_{i j k}(r, t)$ denotes the third order velocity structure 
function tensor, given by

$D_{i j k}(r, t)=\left\langle\left[u_{i}(\boldsymbol{x}+\boldsymbol{r}, t)-u_{i}(\boldsymbol{x}, t)\right]\left[u_{j}(\boldsymbol{x}+\boldsymbol{r}, t)-u_{j}(\boldsymbol{x}, t)\right]\left[u_{k}(\boldsymbol{x}+\boldsymbol{r}, t)-u_{k}(\boldsymbol{x}, t)\right]\right\rangle$.

Just like the second order structure function tensor, which can be related to $D_{L L}(r, t)$ alone in the case of isotropic turbulence, $D_{i j k}(r, t)$ can be expressed through the longitudinal third order structure function $D_{L L L}(r, t)$ Monin \& Yaglom, 1975; Argyris et al., 2010):

$$
D_{i j k}(r, t)=\frac{1}{6} \frac{\partial}{\partial r}\left(r D_{L L L}(r, t)\right)\left[\delta_{i j} \frac{r_{k}}{r}+\delta_{i k} \frac{r_{j}}{r}+\delta_{j k} \frac{r_{i}}{r}\right]-\frac{1}{2} r^{2} \frac{\partial}{\partial r}\left(\frac{1}{r} D_{L L L}(r, t)\right) \frac{r_{i} r_{j} r_{k}}{r^{3}}
$$

The derivation of the Kolmogorov equation is continued by setting $i=j$ in Eq. 2.18 in order to find an expression for $\frac{\partial}{\partial t} D_{i i}(r, t)$. Using the relations Eq. 2.12), Eq. 2.13 and Eq. 2.20 and noting that the energy dissipation rate can be written as (Davidson, 2004)

$$
\epsilon=\nu\left\langle\frac{\partial v_{i}}{\partial r_{k}} \frac{\partial v_{i}}{\partial r_{k}}\right\rangle
$$


one finds

$$
\frac{\partial D_{i i}(r, t)}{\partial t}=-\frac{1}{r^{2}} \frac{\partial}{\partial r}\left[\frac{1}{3 r} \frac{\partial}{\partial r}\left(r^{4} D_{L L L}(r, t)\right)\right]+\frac{2 \nu}{r^{2}} \frac{\partial}{\partial r}\left[\frac{1}{r} \frac{\partial}{\partial r}\left(r^{4} \frac{\partial}{\partial r} D_{L L}(r, t)\right)\right]-4 \epsilon
$$

A relation between $\frac{\partial}{\partial t} D_{i i}(r, t)$ and $\frac{\partial}{\partial t} D_{L L}(r, t)$ can be obtained by setting $i=j$ in Eq. 2.12 and additionally replacing $D_{N N}(r, t)$ according to Eq. 2.13. Deriving it with respect to time and solving for $\frac{\partial}{\partial t} D_{L L}(r, t)$, one then finds

$$
\frac{\partial}{\partial t} D_{L L}(r, t)=\frac{1}{r^{3}} \int_{0}^{r} s^{2} \frac{\partial}{\partial t} D_{i i}(s, t) d s
$$

Finally, by replacing $\frac{\partial}{\partial t} D_{i i}(r, t)$ in Eq. 2.23 by Eq. 2.22 and working out the integral, one arrives at the Kolmogorov equation

$$
\frac{\partial}{\partial t} D_{L L}(r, t)=-\frac{1}{3 r^{4}} \frac{\partial}{\partial r}\left(r^{4} D_{L L L}(r, t)\right)+2 \nu \frac{1}{r^{4}} \frac{\partial}{\partial r}\left(r^{4} \frac{\partial}{\partial r} D_{L L}(r, t)\right)-\frac{4}{3} \epsilon
$$

The Kolmogorov equation shows the temporal evolution of the longitudinal second order structure function $D_{L L}(r, t)$ and is derived from the Navier-Stokes equations without any further modeling except the assumption of homogeneity and isotropy. It is the starting point of numerous theoretical work afterwards. But it also shows another problem that we have to face when describing turbulence theoretically. As one could already see in Eq. (2.18), the system of equations is not closed as 
the third order structure function appears in the equation of motion of the second order structure function. If one now tries to derive an equation for the temporal evolution of $D_{L L L}(r, t)$ to close the system, the fourth order structure function will appear in that equation and so on. This is called the closure problem of turbulence. To find an appropriate closure model is the center of attention in many studies (Pope, 2000).

Based on Eq. (2.24), Kolmogorov argued, that for scales in the inertial range the time derivative is zero due to the statistical steadiness of the small scales and that the second term on the right hand side becomes negligible compared to the first. This leads to what is known as Kolmogorov's 4/5-Law

$$
D_{L L L}(r, t)=-\frac{4}{5} \epsilon r
$$

\subsection{Perturbing a Turbulent Flow}

Kolmogorov's theory, presented above, relies on the steadiness of the statistical quantities. This is reached in turbulent flows with constant energy input. In this

case, the predictions compare well to experiments and simulations (Pope, 2000).

In natural and technical flows, however, the energy input is not always constant. Eq. (2.24) shows that theoretical predictions for time-dependent statistics can only 
be made if further assumptions for a closure are made. It is therefore important to investigate turbulent flows with varying energy input in order to understand their dynamics. In the following, previous work on varying energy input is reviewed.

In a turbulent flow with a forcing consisting of periodically occurring pulses (kicks), the dependence of the turbulent kinetic energy on the frequency of these kicks was investigated theoretically (Lohse, 2000), numerically (Hooghoudt et al. 2001) and experimentally (Jin \& Xia, 2008).

Cadot et al. (2003) investigated modulated turbulence in a von Kármán flow, i.e. the propellers forcing the flow followed a sinusoidal velocity profile. To quantify the response to the modulated large scale forcing, the local turbulent kinetic energy was measured. They found that for small modulation frequencies $f$ the turbulent kinetic energy followed the modulation and the response amplitude stayed constant, while for larger frequencies a phase shift of the response was observed and the response amplitude decreased with $1 / f$. From the crossover point of these two regimes the turbulent cascade time was inferred. Moreover, for certain modulation frequencies response extrema were observed. In these cases, the velocity fluctuations were stronger than in the unmodulated case with the same average energy input. This suggests that a more efficient mixing can be achieved by modulating the forcing of a turbulent flow.

In a theoretical study published around the same time von der Heydt et al. 
$2003 a$ ) for a modulated energy input rate, the $1 / f$ decay of the response as well as the appearance of response extrema was predicted. The authors used a meanfield theory approach to derive a closure for Eq. 2.24) and assumed a time delay between energy input and dissipation, the cascade time. The predicted behavior could also be recovered in numerical studies von der Heydt et al., 2003b; Kuczaj et al., 2006; Bos et al., 2007; Kuczaj et al., 2008) and other experiments (Cekli et al. 2010). Further experimental studies of small scale response to large scale perturbations can be found in Camussi et al. (1997); Labbé et al. (2007); Hamlington \& Dahm (2009); Chien et al. (2013).

While the work mentioned above is fairly recent, investigations on pulsating pipe flow seem to have a longer history (Gerrard, 1971; Tu \& Ramaprian, 1983; Ramaprian \& Tu, 1983; He \& Jackson, 2009; He \& Seddighi, 2013).

Investigations on the time it takes for the energy of the large scales to be transferred to the smallest scales of turbulence, the cascade time, were presented by Pumir (1996); Pearson et al. (2004); Meneveau \& Lund (1994)

A very traditional field of turbulence research without a constant energy input focuses on the decay of turbulence (Batchelor, 1953, Saffman, 1967a b; ComteBellot \& Corrsin, 1971; Stalp et al., 1999; George, 1992; Ishida et al., 2006, Lavoie et al., 2007; Teitelbaum \& Mininni, 2009, 2011; Krogstad \& Davidson, 2010; Sinhuber et al. 2015) 
The scope of the work presented in this thesis lies on the investigation of homogeneous and isotropic turbulent flows that are subject to an abrupt change of the large-scale forcing, i.e. an increase of energy input in one case and a cut off of the energy input in the other case. In comparison to the previously mentioned investigations, the data presented in this thesis allow an insight in the scale-dependent response of a turbulent flow under varying large-scale forcing. 


\section{Chapter 3}

\section{Experimental Setup}

In this Chapter the experimental setup, used to measure the scale dependent response of the energy transfer in a homogeneous and isotropic turbulent flow subject to a temporal change of the energy injection, is described. The apparatus, used to produce such a flow, is introduced in Section 3.1. The measurements were performed with Lagrangian Particle Tracking (LPT), a non-invasive optical measurement technique, described in Section 3.2. In the LPT system several highspeed cameras are used in order to observe the movement of tracer particles! 1 in the measurement volume of the apparatus from different angles. As the size of the internal RAM (Random Access Memory) of the cameras limits the duration of a measurement a real-time image compression system was developed to overcome

\footnotetext{
${ }^{1}$ Particles that faithfully follow the flow.
} 
this limitation. This system is presented in Section 3.3. A description of the Laser used for the measurement volume illumination can be found in Section [3.4. An overview over how the whole setup works together and its automation, is given in Section 3.5.

\subsection{Lagrangian Exploration Module}

The apparatus used to obtain the data presented in this work, the Lagrangian Exploration Module (LEM), is an icosahedron shaped water container with 12 propellers, one on each vertex (Fig. 3.1). Each triangular face has an edge length of $40 \mathrm{~cm}$, which gives a diameter of the icosahedron of about $1 \mathrm{~m}$ and a volume of $140 l$. In this section, a summary of the most important specifications of the LEM is given. A more detailed description of the LEM and the flow field it produces can be found in Zimmermann (2008) and Zimmermann et al. (2010).

The skeletal structure of the Lagrangian Exploration Module consists of stainless steel. Out of the 20 faces of the icosahedron, 18 are covered with Plexiglas windows for optical access. The top and bottom faces are covered with stainless steel cooling plates to maintain a stable temperature of the experimental fluid. The cooling plates are flushed with $10^{\circ} \mathrm{C}$ cooling water from the building supply and the flow rate is adjusted manually using a ball valve. 

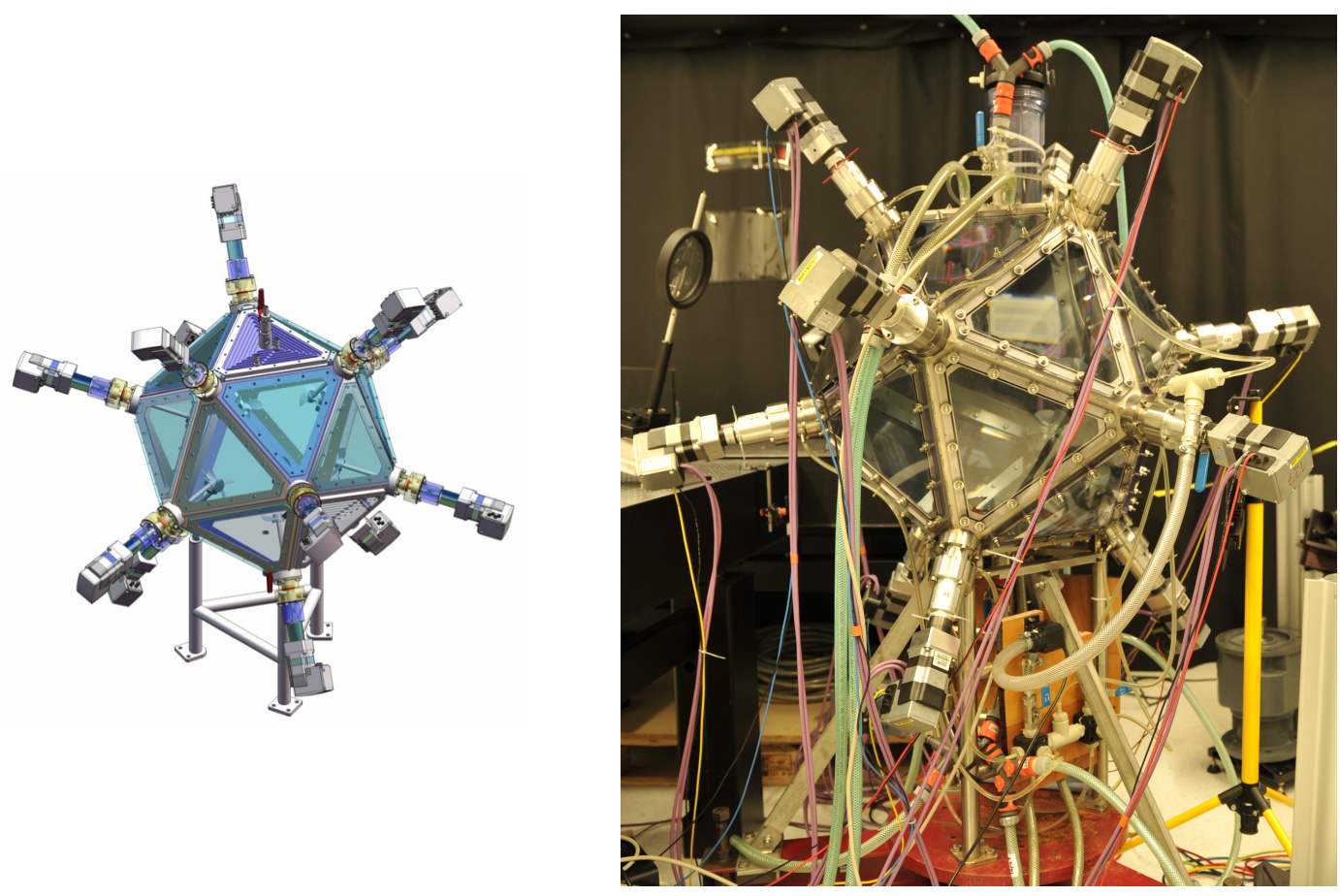

Figure 3.1: The Lagrangian Exploration Module (LEM) is an icosahedron shaped container. On each vertex a motor is installed to rotate a propeller inside the container. The mechanical drawing on the left was taken from Zimmermann (2008).

On each of the 12 vertices a brushless DC motor (IFE71 by Berger-Lahr) is installed and drives a propeller inside the LEM through a planetary gear box with a fixed speed ratio of $5: 1$. While the motor speed can be set between 300 and $5000 \mathrm{rpm}$ (revolutions per minute) the propeller speed is reduced accordingly to a value between 60 and $1000 \mathrm{rpm}$. Both, clockwise and counterclockwise rotation are possible. Rotation rates mentioned in the rest of the thesis will refer to the 
propeller speed if not stated otherwise.

The required DC voltage of $36 \mathrm{~V}$ for the motors is provided by two DC power supplies (N5766A by Agilent). All motors are, together with a computer, connected by a CANopen $2^{2}$ bus network and can be controlled individually. Using a custom software ${ }^{3}$ and an Ethernet to CAN gateway (AnaGate CAN by Analytica), the computer controls the motors and monitors parameters like current, temperature, velocity etc.

To investigate the development of a turbulent flow under a sudden change in the energy injection, i.e. a change in motor speed, the moment when the motor speed changes has to be known as exactly as possible. Therefore the supply current of one motor was used as an indicator for the change in speed of all motors. For this purpose, a comparator circuit, developed by Ortwin Kurre, measures the current of one of the motors of the LEM (Fig. 3.2), compares it to a threshold value set with a potentiometer, and switches on a laser pointer once the current surpasses the threshold. The laser pointer produces a bright spot on the sensor of one of the cameras, such that the change of motor speed can be determined from the same high-speed recordings used to perform LPT.

As the working fluid, deionized water from the building supply was used for all

\footnotetext{
${ }^{2}$ The Controller Area Network (CAN) is a communication protocol developed for networks of embedded systems.

${ }^{3}$ The software was written by Robert Zimmermann and Shinji Tanaka. The author embedded parts of this software in another program for automation of the experiment (Section 3.5).
} 


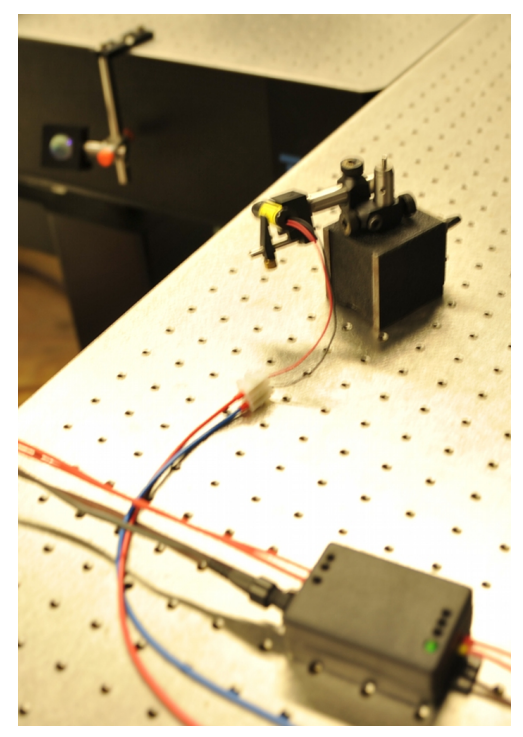

Figure 3.2: A comparator circuit (lower right corner) measures the supply current of one motor of the LEM and compares it to a threshold that can be adjusted with a potentiometer. If the motor speeds up and its current exceeds the threshold, a relay is energized and switches on a laser pointer (center). This laser pointer is guided to the sensor of one of the cameras via a mirror (upper left corner) and produces a bright spot in the high speed recording, such that the exact moment of the change of motor speed can be determined.

experiments presented in this thesis. After filling the LEM, the deionized water was filtered to remove the leftover particles of previous LPT measurements and dust particles that entered the apparatus through the water supply lines. For the filtering, a pump was used to circulate the water from the bottom of the LEM 
through two successive filters (pore sizes are $10 \mu m$ and $5 \mu m$ ) and returning to the top, see Fig. 3.3 (a). Filtering everything larger than $5 \mu m$ was sufficient for our purpose, as tracer particles with an average diameter of $80 \mu m$ were used. The filtering procedure usually took about 24 hours.

When filling the LEM, usually some air bubbles are trapped in the vertices and some stick to the windows. There is also air trapped in the filtering circuit. Therefore, after filtering, a degassing procedure explained in Zimmermann et al. (2010) was followed. At each vertex of the LEM, close to the rotating seal where bubbles are trapped, there is a through-hole for air to escape. These are connected to a degassing circuit, as shown in Fig. 3.3 (b), where the water is pumped from the six upper vertices through a small container, where the air bubbles rise to the top and escape the circuit, to the six lower vertices. It was found by Zimmermann et al. (2010) that the degassing procedure is more efficient if always two opposing propellers are switched on and off randomly. For this purpose, a special degassing motor control scheme, implemented in the motor control software by Robert Zimmermann, was used. After a few hours of degassing the deionized water was free of air. Only after the filtering and degassing procedures, tracer particles were added to the flow. 


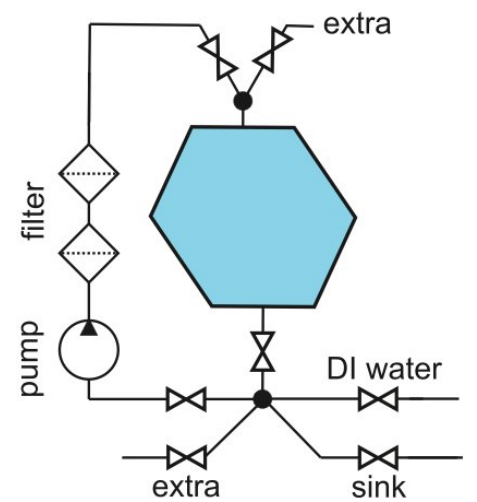

(a) Filtering circuit

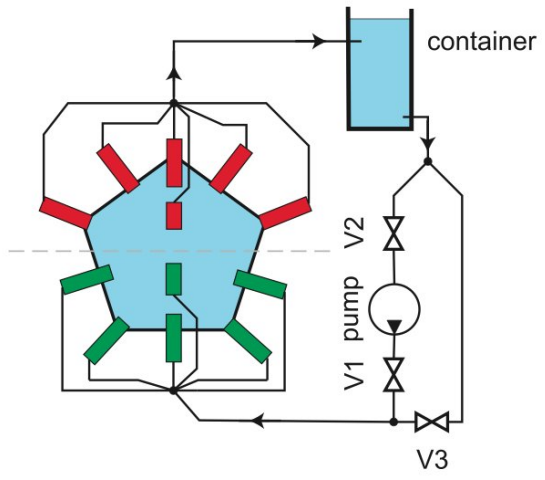

(b) Degassing circuit

Figure 3.3: (a) Filtering circuit: A pump circulates the deionized water through two filters, with pore size of $10 \mu m$ and $5 \mu m$, respectiveley. Two extra connectors are provided, but are not used in this work. (b) Degassing circuit: A pump circulates the water from the upper six bubble traps through a container that allows for gas to escape before the water returns to the lower six bubble traps. The valves V1, V2 and V3 allow a by-passing of the pump, but this function was not used in this work. Both images, (a) and (b), are taken from Zimmermann (2008). 


\subsection{Lagrangian Particle Tracking}

When describing a flow field, two viewpoints can be taken: the Eulerian or the Lagrangian point of view. In the Eulerian framework, one focuses on a certain point in space and describes the velocity at that point. Corresponding measurements are therefore called Eulerian measurements. Typical techniques are Laser Doppler Velocimetry, Particle Image Velocimetry and hot-wire measurements. In these cases, the velocity of a flow is measured as a function of time at fixed positions in space. In contrast to the Eulerian framework, a fluid flow can also be described in the Lagrangian framework (Yeung, 2002; Toschi \& Bodenschatz, 2009). In this view, the velocity field is described along the trajectories of fluid particles as they are moving in the flow. The measurement technique used in this work belongs to this category and is called Lagrangian Particle Tracking (LPT). One advantage of LPT is that the obtained data can be interpreted from the Eulerian point of view as well. The data presented in this thesis are based on analysis of Eulerian statistics.

The basic idea of LPT is to seed the working fluid with particles and to use cameras to measure the position of each particle as a function of time to obtain Lagrangian trajectories (Snyder \& Lumley, 1971; Dracos, 1996; Mann et al., 1999). The cameras, typically three or four ${ }^{4}$, are set up to focus on the measurement vol-

\footnotetext{
${ }^{4}$ The setup presented in this thesis has four cameras, three are used for LPT, the fourth
} 
ume from different angles in order to obtain particle positions in three dimensions (3D). The cameras are synchronized, such that all cameras take images at the same time. To obtain 3D particle tracks in lab space we follow the algorithm presented in Ouellette et al. (2006a) and $\mathrm{Xu}$ (2008). In the following, the setup and calibration of the cameras are described and the basic steps of the LPT procedure are outlined.

\subsubsection{Camera Setup and Calibration}

For this experiment, four Phantom V640 high-speed cameras were set up and aligned to observe the center of the LEM (Fig. 3.4). Three of those cameras, Camera 0 through to Camera 2, were used to perform Lagrangian Particle Tracking. The fourth camera was used to record the signal of the laser pointer, indicating when the change of motor speed occurs. All cameras were controlled by the same computer and were fed with an external square wave signal to record images at the same time and with the same frame rate (Section 3.5).

The three cameras used for LPT record the movement of particles in the measurement volume of the LEM. To be able to reconstruct the position of each particle, detected in the 2D sensor plane of each camera, in 3D lab space we followed a calibration method described in Tsai (1987) and Ouellette et al. (2006b). In camera is used to detect the moment of change in motor speed. 


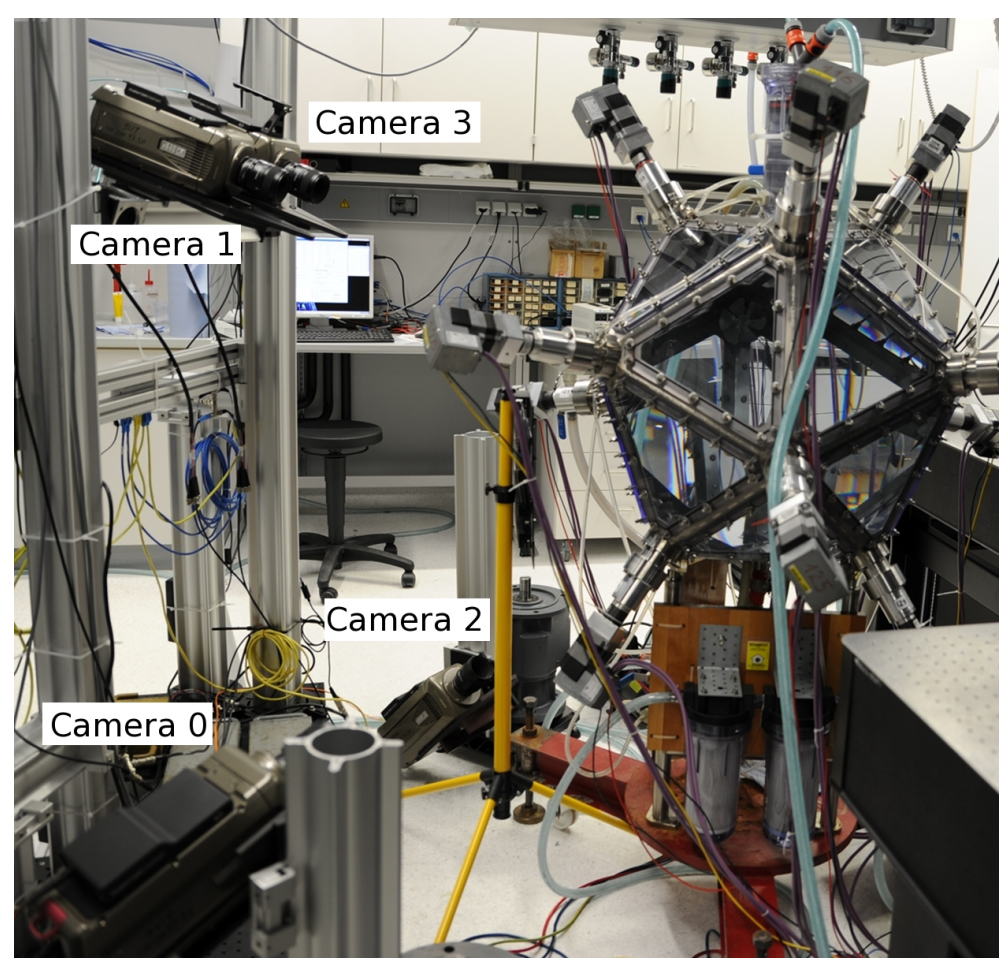

Figure 3.4: The camera setup. The cameras 0 through to 2 are aligned to the measurement volume in the center of the LEM and used for LPT. Camera 3 is used to capture when the laser pointer, indicating the change of motor speed, is switched on.

order to do this, a mask with a regular dot pattern of known spacing $(1.016 \mathrm{~cm})$ was inserted in the center of the LEM (Fig. 3.5). The mask provided dots in two perpendicular directions ( $\mathrm{y}$ and $\mathrm{z}$ ) of lab space. A translation stage on the mask holder was used to accurately displace the mask in the $\mathrm{x}$ direction. Then, image sequences of the mask at various positions along the $\mathrm{x}$ axis (every $1 \mathrm{~cm}$ ) were 


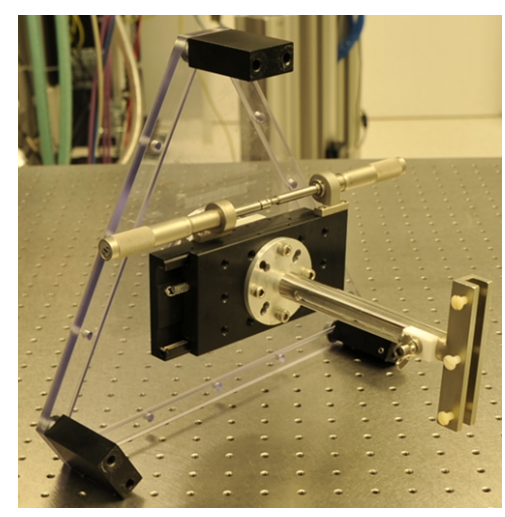

Mask holder

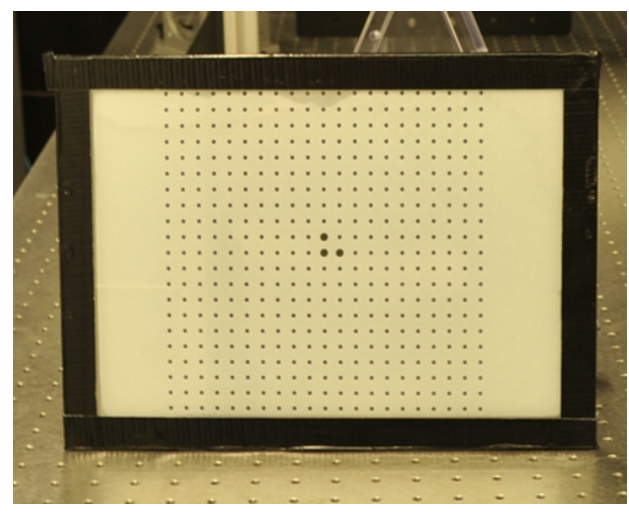

Mask

Figure 3.5: Mask and holder for the calibration of the cameras used for LPT. After removing the top plate of the LEM, the mask holder was placed on the LEM such that it held the mask in the center of the tank. The mask provided dots with a defined spacing in $\mathrm{y}$ and $\mathrm{z}$ direction. A translation stage mounted to the holder was used to move the mask in the $\mathrm{x}$ direction.

recorded. As an example, Fig. 3.6 shows the mask at the center position. With this information, the camera positions and orientations were determined in lab space using a program written by Haitao Xu. After this procedure, for a particle detected in the 2D plane of a camera sensor a line of possible positions through the measurement volume, the line of sight, could be calculated. It is described in the sections below, how the information of all cameras was used to determine the 3D position of a particle. 


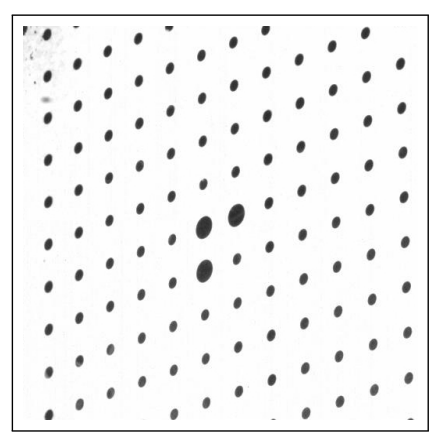

Camera 0

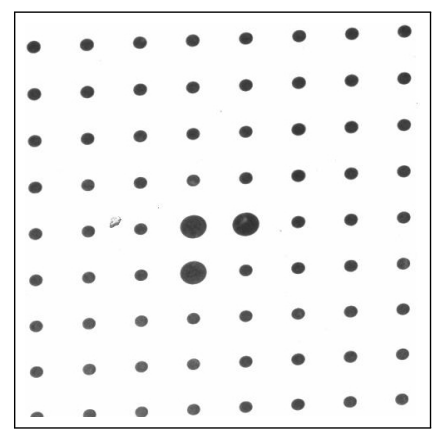

Camera 1

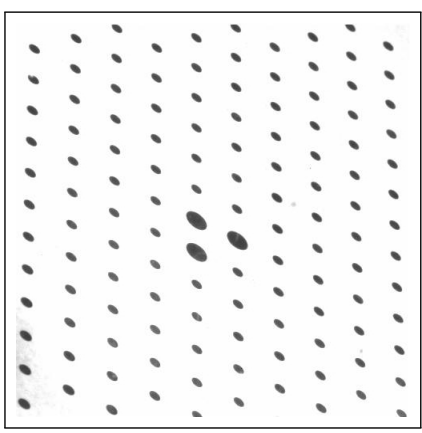

Camera 2

Figure 3.6: Calibration images of the cameras used for LPT, taken with the mask in the center of the tank.

\subsubsection{Particle Finding}

The first step was to find all particles in every image and to determine their $\mathrm{x}$ and y coordinates on the image planes of the cameras. To do this, every pixel in every frame is compared to a threshold 5 . While this is usually done in the post processing, we developed a system to perform the thresholding in real time, during data acquisition (Section 3.3). Only pixels brighter than this threshold are considered to represent particles, those pixels with an intensity lower than the threshold are considered to be the background, which is usually noisy. The pixels that pass the thresholding typically form groups of connected pixels. Fig. 3.7 shows

\footnotetext{
${ }^{5}$ The threshold has to be determined in an iterative approach before the experiment and can be different for each camera.
} 
a section of a typical image after thresholding. Now the center of each group of pixels is determined with sub-pixel resolution by fitting Gaussian functions to the intensity distribution (Ouellette et al., 2006a). This procedure is done for every frame of every camera independently.

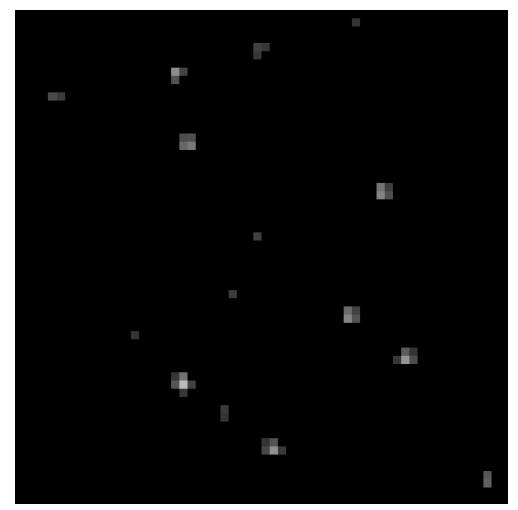

Figure 3.7: A 60 by 60 pixels section of a typical image of LPT after thresholding. The center of every group of pixels is determined with sub-pixel resolution by fitting a Gaussian function to the intensity distribution (Ouellette et al., 2006a).

\subsubsection{Stereoscopic Matching}

Now that the particle positions on the 2D image plane of each camera at each time step are known, the 3D position of each particle in lab space at each time step has to be obtained. This second step of the particle tracking algorithm is called stereoscopic matching. To understand how it works a setup with only two cameras 
is considered.

As shown in Fig. 3.8 the $2 \mathrm{D}$ information about the position of a particle on the image plane of camera $\mathrm{A}$ is used to calculate a line of sight through the measurement volume. This line of sight of camera A is then projected on the image plane of camera B. If a particle center on the image plane of camera B falls on that projected line ${ }^{6}$ then a match is found. The $3 \mathrm{D}$ position of the particle can then be obtained from the intersection of the two lines of sight from cameras A and B. In reality, a particle center on camera B will never exactly fall on the line projected from the line of sight of camera A. Therefore a small tolerance is used and particles within this tolerance are considered matches. It can happen, that two particle centers on camera B are possible matches for the particle center on camera $\mathrm{A}$. Therefore the use of three or more cameras is favorable. As a requirement for the stereoscopic matching, the positions and orientations of the cameras in lab space need to be determined. In order to do this a calibration method described in Tsai (1987) and Ouellette et al. (2006b) was followed.

\footnotetext{
${ }^{6}$ There is not necessarily a particle center falling on that line, as not all particles are detected by all cameras.
} 


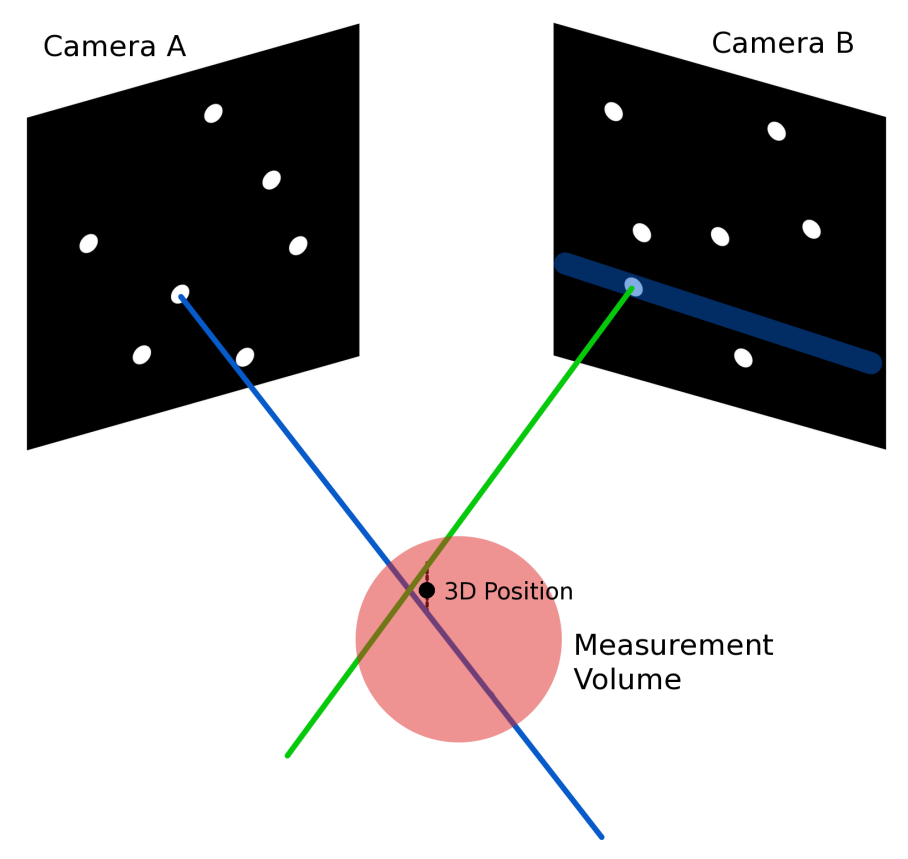

Figure 3.8: Stereoscopic matching with two cameras. Starting with a particle on the image plane of camera $\mathrm{A}$ and calculating its line of sight to the measurement volume (blue line). The line of sight is then projected on the image plane of camera B with a small tolerance (blue shade). The particle found in the projection region of camera B is considered to be the match for the particle from camera $\mathrm{A}$ and its line of sight is calculated (green line). Since the lines of sight do not exactly intersect, the 3D position is considered to be the position with the smallest distance to all lines of sight. 


\subsubsection{Particle Tracking}

The final step that has to be performed is the particle tracking in time. In this step, the 3D particle positions from all time steps are used to build particle trajectories. To do this, every individual particle (in 3D lab space) that has been found in one time step $n$ has to be identified amongst those particles found in the consecutive time step $n+1$. Different algorithms, together with their advantages and disadvantages for particle tracking, are discussed in Ouellette et al. (2006a). The algorithm applied to the experimental data gathered in the scope of this thesis is briefly described in the following.

When starting a new track the nearest neighbor approach is used. Consider a particle at the position $\boldsymbol{x}_{n}$ in time step $n$. This individual particle has to be identified in time step $n+1$. Therefore, from all particles found in a search radius around $\boldsymbol{x}_{n}$ in time step $n+1$ the particle closest to $\boldsymbol{x}_{n}$ is chosen. 


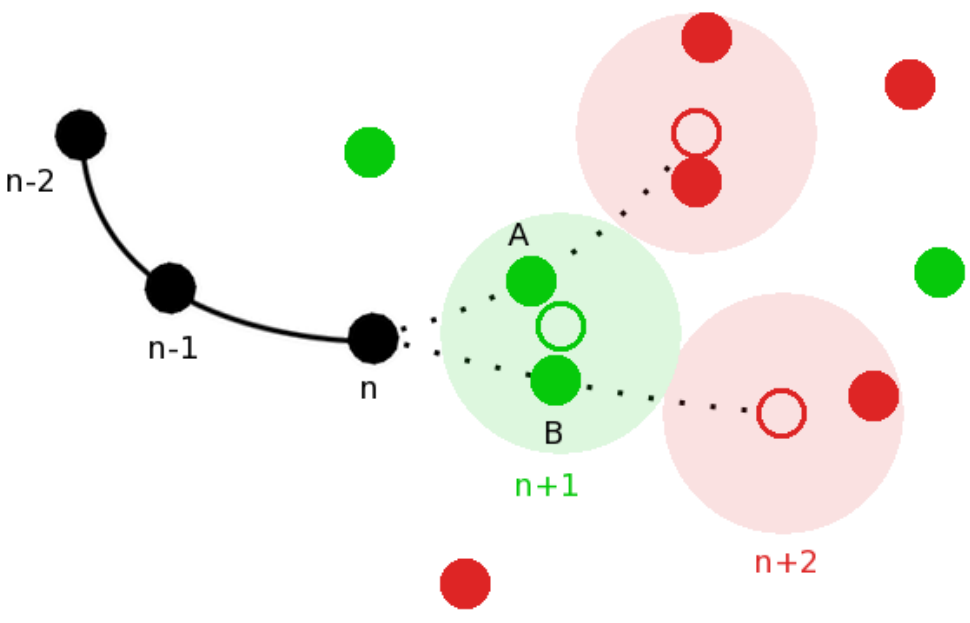

Figure 3.9: Illustration of the particle tracking method best estimate. Note that particles of different time steps are shown. Solid black line with black circles: a particle track with known particle positions at time steps $n-2, n-1$ and $n$. Filled green circles: available particle positions at time step $n+1$. Filled red circles: available particle positions at time step $n+2$. The known particle positions at times $n-1$ and $n$ are used to calculate a velocity and estimate its position at time step $n+1$ (open green circle). Then the particles A and B, found in a search radius (green shaded area) are used, together with the known particle positions at times $n-1$ and $n$ to calculate the accelerations and estimate the positions at time step $n+2$ (open red circles). Depending on which particle within the search radius (red shaded areas) at time $n+2$ is the closest to an estimated position, either $\mathrm{A}$ or $\mathrm{B}$ is chosen as the continuation of the track. In this example, A continues the track. 
After having a track with at least two positions, the best estimate algorithm is used. This algorithm is depicted in Fig. 3.9. The starting point is the track we assembled so far, shown as a black solid line with the positions of the particle at time steps up to $n$. The two positions at time $n-1$ and $n$, are used to calculate the velocity of the particle and estimate its position at time step $n+1$ (open green circle). Now all particles found at time step $n+1$ (green circles) within a search radius (green shaded area) around the estimated position are considered possible candidates to continue the track. In the example in Fig. 3.9 two particles, A and $\mathrm{B}$, are within the search radius. To decide which continues the track one more step is necessary. From the particle positions at $n-1, n$ and each possible position at $n+1$, the particle acceleration is calculated and used to estimate the particle position at $n+2$ (open red circles). In a search radius (red shaded area) around each estimated position at $n+2$ we find the particle (red circles) closest to one of the estimated positions. This is then used to decide which particle at time step $n+1$ continues the track. In Fig. 3.9 particle A would be chosen. The algorithm then repeats to determine the position of this particle at time step $n+2$ by using information of known particle positions at time steps $n$ and $n+1$ and available particle positions at times $n+2$ and $n+3$. 


\subsection{Real Time Image Compression System}

High-speed cameras are designed to record videos of processes that are too fast to be seen with the naked eye. This is done by shooting hundreds or thousands of images per second and replaying the recorded video at slow frame rates like $25-50 \mathrm{~Hz}$ to analyze the process. Some typical applications are crash tests, assembly line analysis or slow motion shots for the broadcast of sport events. Recording videos at such high frame rates produces enormous data rates, e.g. $\sim 1 \mathrm{~GB} / \mathrm{s}$ when recording grey scale images with 512 by 512 pixels at $3 \mathrm{kHz}$ with 10 bit intensity resolution. These data rates are much larger than the typical writing speeds of hard disc drives (HDD), which are about $50-100 \mathrm{MB} / \mathrm{s}$. As a consequence, the high-speed cameras are usually equipped with a few $G B$ of RAM (Random Access Memory) to buffer the video. The size of the RAM limits the duration of a high-speed video recording to a few seconds, depending on frame rate and frame size. To download the buffered video to a computer for processing or saving it to an HDD, can take several minutes, depending on the size of the video and the available transfer media. For example, to download and save a $32 G B$ movie from our camera, a Phantom V640 from Vision Research, to a regular computer via Ethernet takes about 15 minutes.

Both the short recording times and the relatively long downloading times might not be very problematic for many applications in which the events one is inter- 
ested in are short and rare. In Lagrangian Particle Tracking, however, these two limitations have significant effects. Let us first consider the short duration of the recording. The smallest time scale in a turbulent flow is given by the Kolmogorov time scale $\tau_{\eta}$. The frame rate of the cameras is typically set at $\sim 30 \mathrm{frames} / \tau_{\eta}$ in order to accurately resolve the turbulent dynamics $(\mathrm{Xu}, 2008)$. Since $\tau_{\eta}$ is on the order of $m s$, the frame rate of the cameras has to be on the order of $k H z$. As mentioned before, the trade-off for high frame rates is the video duration. That means, to resolve the fastest time scales one is restricted to short videos for cameras with given internal memory size. In short videos it is difficult to detect slow processes in turbulence that act on time scales longer than the recording. In this work, we are interested in the response of the energy cascade to a perturbation in the energy input. It was not clear a proiri on which time scales this response would occur and it would have been disadvantageous to be limited to short LPT measurements.

Now we want to discuss the effects of the downloading times on LPT measurements. Usually, not enough data points for converging statistics can be retrieved from one high-speed recording due to its limited duration. Therefore, for one LPT experiment many videos have to be taken and an ensemble average is performed. How many videos are needed depends on the experiment and which quantity is measured, but very often several hundred videos are necessary. Considering the 
relatively long downloading times, this means that the conditions for one experiment (temperature, water quality, alignment of the cameras, etc.) have to be maintained for several days or even weeks. In a small laboratory experiment, where the experimentalists have control over the conditions, this is not more than an inconvenience. But there are experiments where long downloading times are drastically reducing the efficiency in terms of data per day. Examples are experiments with high operational costs, like LPT in a wind tunnel as planned in the Göttingen Turbulence Facility (Bodenschatz et al., 2014), or experiments in nature where the experimentalists cannot control the conditions, like the planned LPT experiments in clouds in the Environmental Research Station Schneefernerhaus on Zugspitze (Risius, 2012; Risius et al., 2015; Siebert et al., 2015).

To overcome the issues of short recording times and long downloading times, we decided to follow an approach pioneered by Chan et al. (2007). The basic idea is that if one can stream and compress the image sequence from the high-speed camera to a computer in real time, the videos can be written to a hard disk drive directly. As an example, the data rate of a high-speed recording with $512 \times 512$ pixels and a 10 bit greyscale at $3 \mathrm{kHz}$ is about $1 \mathrm{~GB} / \mathrm{s}$. As we have argued before this cannot be written to HDD directly. But let us consider the data rate of the information we are interested in.

The images in LPT consist of sparse bright spots, the particles, on a black 
background (Fig. 3.7). As explained in Section 3.2.2 the very first step in the post processing is to threshold the image to find bright pixels that represent particles. Let us therefore estimate the data rate of the bright pixels. According to Ouellette et al. (2006a), the stereo matching works efficient for up to 300 particles per image. If we assume each particle to be represented by 5 pixels we have 1500 bright pixels per image. Together with the 10 bit greyscale value, we also need to save the position of each pixel, which can be done with an 18 bit number in case of a $512 \times 512$ pixels image. So each of the 1500 pixels can be represented by a 28 bit number. In our example, with a frame rate of $3 \mathrm{kHz}$ this would result in a data rate of $1500 \cdot 28 \mathrm{bit} \cdot 3 \mathrm{kHz}=15 \mathrm{MB} / \mathrm{s}$ which is well below the writing speed of standard HDDs.

Based on this idea, Chan et al. (2007) developed a circuit which receives the high-speed video stream of the camera at a data rate of $625 \mathrm{MB} / \mathrm{s}$ and compares every pixel with a threshold in real-time (Fig. 3.10). Only those pixels brighter than the threshold and their positions are then sent to the frame grabber card of a computer and written to the HDD. The system achieves a compression factor 7 of $100-1000$ and establishes a real-time data stream from the high-speed camera to the HDD of the computer. Due to the size of the HDD $(600 G B)$ and the fact that the data is compressed they achieve recording times up to a week while the

\footnotetext{
${ }^{7}$ The compression factor is the ratio of the file sizes of the uncompressed video to the compressed video.
} 


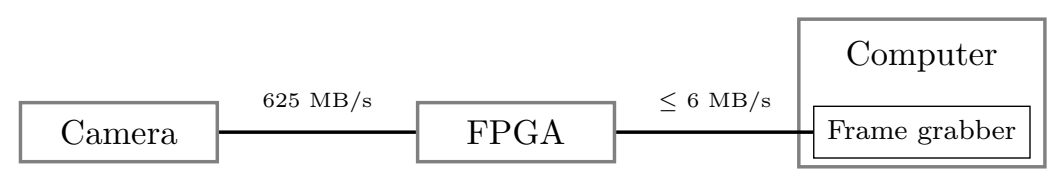

Figure 3.10: Chan et al. (2007) developed an FPGA circuit that receives the camera output, compares every pixel to a threshold and only sends those pixels brighter than the threshold to a frame grabber card. The data rate is reduced by a factor of 100-1000 and therefore the compressed image data can be streamed to the HDD of the computer in real-time.

recording to the $4 G B$ RAM of their camera is limited to about $6 \mathrm{~s}$.

The heart of the circuit developed by Chan et al. (2007) is an FPGA (FieldProgrammable Gate Array), i.e. an integrated circuit allowing the modification of its internal circuit layout and therefore its functionality. The internal circuit layout of an FPGA is called logic and is designed 8 through a Hardware Description Language (HDL). The internal structure of an FPGA is very versatile and allows implementation of nearly any kind of digital circuitry. It is therefore suitable for signal processing and parallelization of processes, and allows for modifications without a physical redesign of the hardware.

Hence, FPGAs are used in a wide variety of applications and one of which is real-time image processing as e.g. in machine vision (Díaz et al., 2006; Jin ${ }^{8}$ Very often the word programming is used in this context. That is misleading, because in fact a digital circuit inside the FPGA is designed. 
et al., 2010). Concerning the particular use of FPGAs for real-time image compression in LPT, only two cases are known to the author. The work of Chan et al. (2007), where an FPGA circuit external to the camera was developed, was already mentioned. Following the same principle of reducing the data rate by discarding information not needed for the LPT post processing, Kreizer et al. (2010) presented a work using an FPGA on board of a high-speed camera. This has the advantage, that the bandwidth of the camera output to the frame grabber can be used more efficiently. Moreover, Kreizer et al. (2010) not only thresholded the image to find the bright pixels but also determined the center of groups of pixels and therefore output 2D particle positions instead of the positions of all pixels that represent a particle. In a later work they combined their camera with a four-view image splitter to perform 3D LPT (Kreizer \& Liberzon, 2011). In the following, our approach towards real-time image compression for LPT using an external FPGA circuit will be described.

\subsubsection{High-speed Cameras}

The high-speed cameras we use are Vision Research's Phantom V640 equipped with the CineStream module (Fig. 3.11). This module provides a fiber optical output ${ }^{9}$ to transmit image sequences at data rates of about $1 G B / s$ (without over-

\footnotetext{
${ }^{9} \mathrm{~A}$ fiber cable with an MTP/MPO connector can be connected. The camera uses 8 out of 12 fibers in the cable with a transmission rate of $2.125 \mathrm{Gbit} / \mathrm{s}$ per fiber.
} 
head). The fiber output can be used to download videos previously recorded to RAM or to transmit a live image stream. In the live streaming case, the bandwidth of the fiber connection reduces the achievable frame rates, compared to those when recording to RAM (Tab. 3.1). All LPT measurements presented in this work were recorded using the live streaming option with $512 \times 512$ pixels at a frame rate of $3 k H z$.

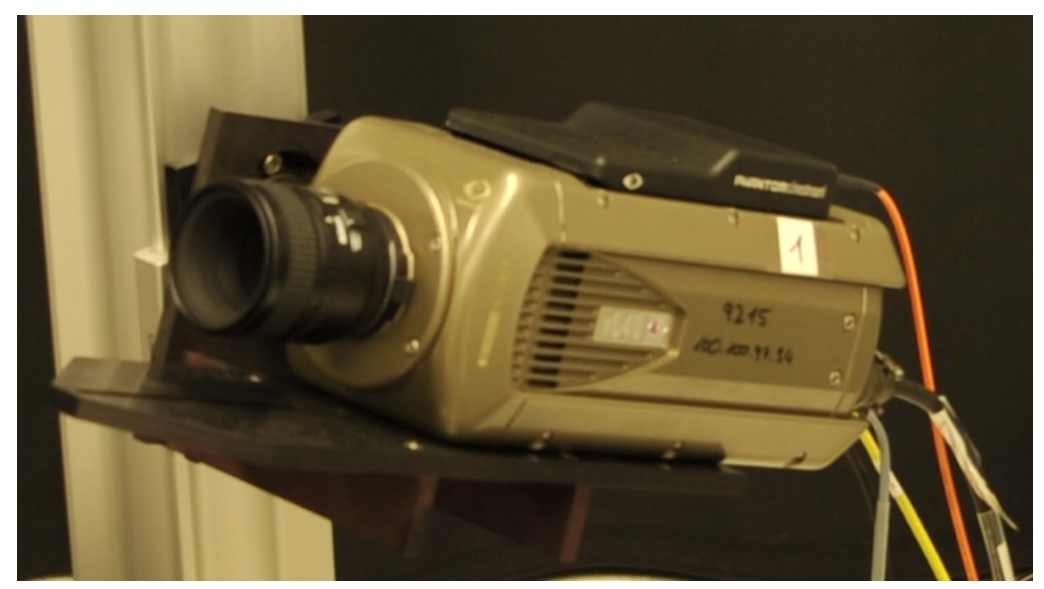

Figure 3.11: Vision Research's Phantom V640 with CineStream module. The CineStream module provides a fiber output to stream high-speed videos continuously at a data rate of about $1 G B / s$.

The Cameras are controlled through software commands for the most part (frame rate settings, exposure time etc.) but also provide input ports for control using digital signals. Two of those are important in this setup. One is the FSYNC input. On this port a square wave signal can be sent and the camera will record an 


\begin{tabular}{||c|c|c||}
\hline $\begin{array}{c}\text { frame } \\
\text { size }\end{array}$ & $\begin{array}{c}\text { frame rate } \\
\text { to RAM (12 bit) }\end{array}$ & $\begin{array}{c}\text { frame rate } \\
\text { through fiber (10 bit) }\end{array}$ \\
\hline \hline $2560 \times 1600$ & 1500 & 195 \\
\hline $1024 \times 1024$ & 4900 & 760 \\
\hline $512 \times 512$ & 15000 & 3000 \\
\hline $256 \times 256$ & 39800 & 12200 \\
\hline
\end{tabular}

Table 3.1: Achievable frame rates of the Phantom V640 when recording to RAM or streaming through fiber. The bandwidth of the fiber $(1 G B / s)$ limits the frame rate.

image with every rising edge. The other external signal is the pre-trigger. A rising edge on this port starts the live stream transmission through fiber. To terminate a transmission the FSYNC signal is switched off. The same signals, FSYNC as well as pre-trigger, are sent to all cameras to ensure they all have the same frame rate and record images at the same time.

It turned out that the cameras do not start the videos at exactly the same time as they are expected even though the pre-trigger signal they receive is from the same source. Measurements showed that delays between the starting times of two cameras can be up to $0.1 \mathrm{~s}$. This imposed a severe problem on synchronization, which was solved by using a unique event in the recording. Therefore, in the 
experiments the recording was started before switching on the Nd:Yag Laser for measurement volume illumination. Hence, the first bright frame of each video was used to temporally align the image sequences recorded from different cameras.

\subsubsection{FPGA System}

To take advantage of the option to receive a continuous data stream of the camera at high frame rates through fiber we developed an FPGA based receiver system. This receiver system was presented in Di Lorenzo (2010) without the real-time compression functionality. This function was implemented in the present work. We therefore developed a real-time image compression system that is comparable to Chan et al. (2007) in architecture as the FPGA is external to the camera and a thresholding of each pixel is performed.

The core of the image compression system is the $X 5$ - $T X$, an FPGA module of Innovative Integration. The X5-TX is mounted in the PCIe slot of a host computer. Since it only provides differential electrical input channels (eSATA), we designed an electrical circuit that transforms the optical signals of the camera to differential electrical signals (Di Lorenzo, 2010). This way we managed to establish a real time data stream from the high-speed camera via fiber cable, and after conversion to electrical signals, into an FPGA card that reduces the data rate and writes the compressed high-speed movie to the hard disc drive of its host computer. 
When transmitting data through the fiber cable, the camera splits up every image on the eight fibers to equal parts $\mathrm{S}^{10}$. We designed a module in the FPGA logic that receives the data of a fiber channel pixel by pixel and compares each pixel to a threshold. This module is implemented eight times, such that the data of all channels can be processed in parallel. The pixels that pass the threshold are, together with their coordinates, assembled into data packets and passed to a module that takes care of the communication with the operating system of the host computer and writes the data to file. This PCIe module was already implemented in the FPGA logic and cannot be modified as it is proprietary of Innovative Integration.

The specific logic design of the PCIe module restricted us to a packet size of $16 \mathrm{~B}$. We therefore decided to use an output file format based on two types of $16 \mathrm{~B}$ packets. One is the frame start packet (FSP) that contains general information (Tab. 3.2). Every time a new frame starts an FSP is issued for every channel. The other type of packet is the data packet (DP). Each DP consists of the channel number from which it was issued and three pixel intensity values with their coordinates (Tab. 3.3). The last DP of a frame may contain less than three pixels and will be filled up with zeros. Note that we obey the byte boundaries when filling the DP, i.e. even though the pixel values are 10-bit numbers and the coordinates are 20-bit

\footnotetext{
${ }^{10}$ The information about how the image is distributed over the eight fiber channels is restricted by a non-disclosure agreement we signed with Vision Research.
} 
numbers, we use $2 \mathrm{~B}$ and $3 \mathrm{~B}$ to store the information in the $\mathrm{DP}$, respectively, to allow byte wise reading of the binary file.

\begin{tabular}{|c|c|c|c|c|c|c|c|}
\hline 1 bit & 7 bits & $1 \mathrm{~B}$ & $2 \mathrm{~B}$ & $3 \mathrm{~B}$ & $6 \mathrm{~B}$ & $1 \mathrm{~B}$ & $2 \mathrm{~B}$ \\
\hline 1 for FSP & Channel ID & Frame number & Line Size & Image Size & all zero & Status & Threshold \\
\hline
\end{tabular}

Table 3.2: Frame Start Packet (FSP)

\begin{tabular}{|c|c|c|c|c|c|c|c|}
\hline 1 bit & 7 bits & 3 B & 2 B & 3 B & 2 B & 3 B & 2 B \\
\hline 0 for DP & Channel ID & Coordinate 0 & Pixel 0 & Coordinate 1 & Pixel 1 & Coordinate 2 & Pixel 2 \\
\hline
\end{tabular}

Table 3.3: Data Packet (DP)

The control software of the FPGA board, provided by Innovative Integration together with the X5-TX, was modified such that a user-defined threshold value can be sent from the GUI ${ }^{11}$ to the FPGA ${ }^{12}$. Moreover the possibility of controlling the FPGA board remotely through network commands sent from a different computer was added by the author. We use this for automation of the experiment and for streaming of the image data to the computer cluster instead of saving them locally on the FPGA's host computer.

\footnotetext{
${ }^{11}$ Graphical User Interface

${ }^{12}$ This function was implemented by Simon Schütz.
} 


\subsection{Nd:YAG Laser}

For illumination of the measurement volume in the center of the LEM a self-built13 frequency doubled Nd:YAG laser (Fig. 3.12) was used. It outputs laser light at a wave length of $532 \mathrm{~nm}$. To increase the output power a Q-Switch was used to pulse the laser rather than using the light of a continuous wave. The repetition rate of the pulsing therefore had to be chosen such that output power was maximized and every frame of the recorded videos would receive the same number of pulses, i.e. the repetition rate had to be an integer multiple of the frame rate of $3 \mathrm{kHz}$. A sufficient mean output power of $\sim 40 \mathrm{~W}$ was reached at a repetition rate of $27 \mathrm{kHz}$ which means that every frame receives nine laser pulses during an exposure.

The beam path can be seen from (Fig. 3.12). After exiting the housing on the right side of the image, the laser beam is collimated with a plano-concave lens with a focal length of $75 \mathrm{~mm}$ and a plano-convex lens with a focal length of $100 \mathrm{~mm}$. Then, the beam is elevated with four mirrors to follow a tilted beam path perpendicular to a window of the LEM. In this tilted part of the beam path, the beam is extended to a diameter of about $10 \mathrm{~cm}$ by two plano-convex lenses with focal lengths of $25 \mathrm{~mm}$ and $400 \mathrm{~mm}$.

\footnotetext{
${ }^{13}$ The laser was built by Jennifer Jucher, Haitao Xu and the author.
} 

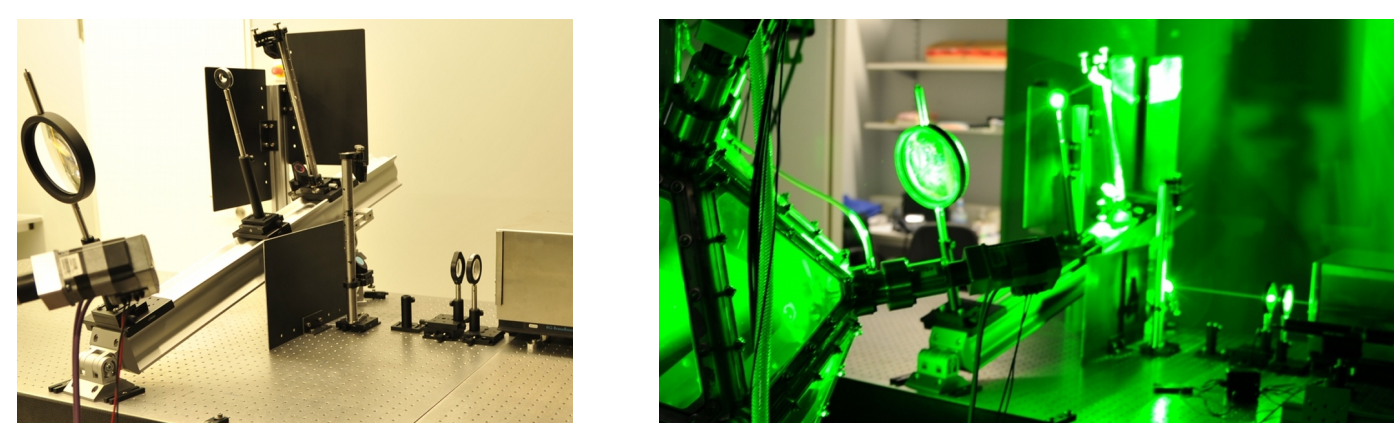

Figure 3.12: The frequency doubled Nd:YAG laser for illumination of the measurement volume. It outputs a mean optical power of $\sim 40 \mathrm{~W}$ at a wave length of $532 \mathrm{~nm}$ and a repetition rate of $27 \mathrm{kHz}$. The laser beam is collimated and then expanded to a size of about $10 \mathrm{~cm}$. It is guided through the measurement volume such that it enters the LEM perpendicular to the Plexiglass window.

\subsection{Automation of the Experimental Setup}

In this section we give an overview on how all the devices involved in the experiments work together to automatically acquire experimental data. The setup involves the LEM, an Nd:YAG laser, the Master Computer and four units of highspeed camera, FPGA computer and cluster node (Fig. 3.13). A frequency generator (FG) provides the laser as well as the cameras with external square wave signals. The cameras are fed with a $3 \mathrm{kHz}$ signal on their FSYNC inputs in order to record images at the same time. The Q-Switch of the laser is fed with a $27 \mathrm{kHz}$ signal, such that every recorded frame is illuminated by nine laser pulses during 
exposure time. The $27 \mathrm{kHz}$ signal is delayed compared to the $3 \mathrm{kHz}$ signal to ensure that the exposure of the camera sensor starts before the first laser pulse is released.

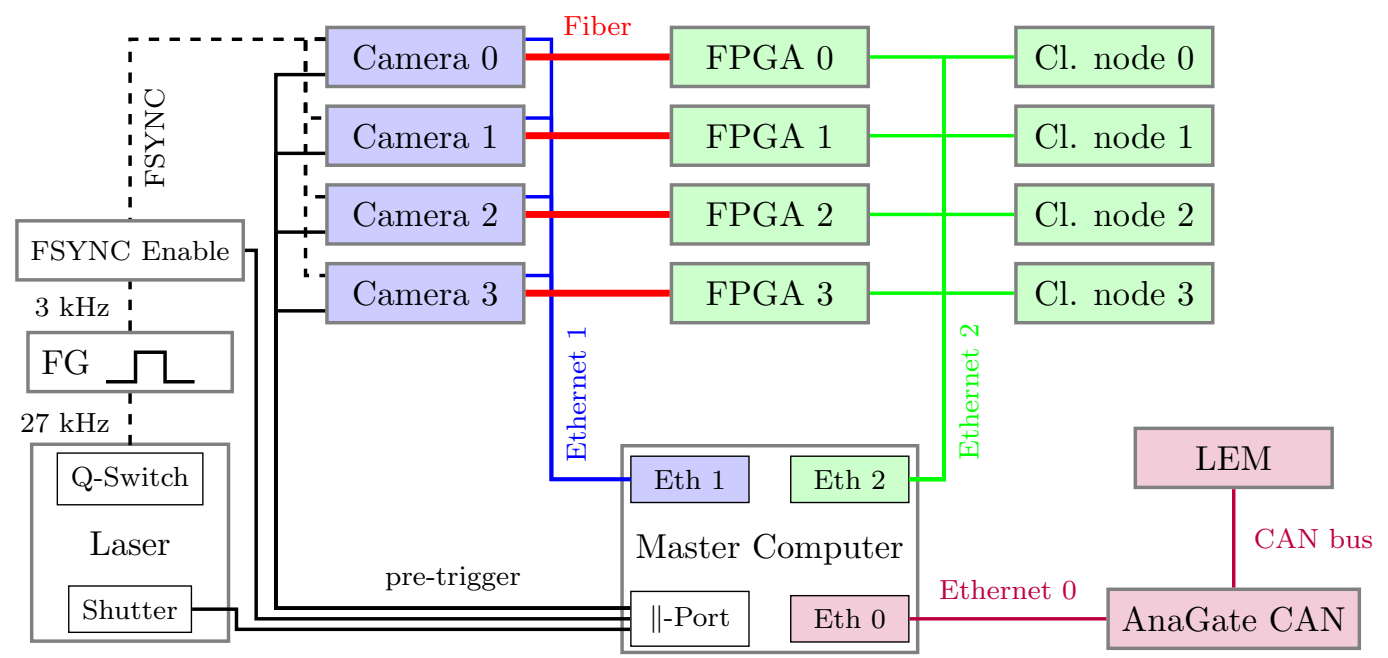

Figure 3.13: A sketch of the network of the setup. Every camera is connected to an FPGA computer via fiber cable. The FPGA computers and the cluster nodes are in an ethernet network. The real-time data stream runs from the cameras to the FPGA computers at a high data rate $(1 G B / s)$, is compressed by the FPGAs and sent to the cluster nodes for saving. The Master Computer controls the LEM, the cameras and the communication between FPGA computer and cluster nodes. With digital signals from the parallel port, the data stream can be started (using pre-trigger) or stopped (using FSYNC Enable) and the laser light can be switched on and off by controlling the shutter of the laser. 
The Master Computer's parallel port is used to generate three important digital signals for the other components. (i) The first signal is used to control the shutter of the laser in order to switch the laser illumination on or off. (ii) The second signal is sent to the cameras as pre-trigger to start the transmission of live image data through the fibers. (iii) The third signal is used to terminate the fiber transmission by interrupting the $3 k H z$ FSYNC signal. This is done using a simple AND gate (FSYNC Enable) with the FSYNC signal of the frequency generator on one input, while the output is connected to the cameras. The parallel port signal on the other input of the AND gate is used to enable or disable its output and therefore the transmission of the square wave.

Since the Master Computer is responsible for the control of the cameras, the FPGA computers, the cluster nodes and the LEM, it resides in three separate Ethernet networks. (i) The motors of the LEM are controlled through Ethernet 0 using an Ethernet to CAN bus converter (AnaGate CAN). (ii) Ethernet 1 connects the Master Computer with the Cameras to transfer settings like frame rate, frame size, exposure time and other control commands. (iii) The FPGA computers and the cluster nodes are connected to the Master Computer through Ethernet 2, for the Master Computer to initiate the establishment of the data stream between each FPGA computer and its respective cluster node, after which it is possible to stream the data from the cameras through optic fibers to the FPGA computers, 
where the data is compressed and forwarded to the cluster nodes for saving.

The program sequence of the main control program on the Master Computer is described in the follwing. The purpose of the main control program is to enable the system to record a specified number of movies, in which the motor speed changes between two user defined values, automatically. The user can define the timing by setting three time values before the start of the experiment. (i) The recording time $t_{r e c}$ specifies the duration of each recorded movie. (ii) Next, a delay time $t_{d e l}$ determines when the change of motor speed from speed 1 to speed 2 occurs. In the control program, $t_{d e l}$ is given in seconds after the start of the recording. (iii) After the recording is finished, the motor speed is again changed from speed 2 to speed 1. To make sure the turbulent flow is stationary again at speed 1 before starting the next recording a waiting time $\Delta t$ between two movies has to be set.

The following description is supplemented by the timing diagram, Fig. 3.14 , and the flow chart, Fig. 3.15. The numbering of the dashed lines in Fig. 3.14 corresponds to the numbering of the boxes in the flow chart (Fig. 3.15). Note that the spacing between the numbered dashed lines in Fig. 3.14 does not represent the real time span between the respective steps. 
(1) When the program starts, important parameters are set in the LEM, like acceleration ${ }^{14}$, and sent to the FPGA computers, such as recording time $t_{\text {rec }}$, and to the cameras (frame rate, frame size, exposure time etc.). After each device confirms the receiving of the message, FSYNC is enabled to provide the cameras with the $3 k H z$ square wave signal.

(2) In the second step, the cycle starts. At first, parameters like frame rate, frame size, exposure time etc. are sent to a server progran ${ }^{15}$ running on the cluster, which then writes these parameters in the header of the data file. Then the propellers are set to speed 1 and the program waits for $\Delta t$ to make sure the flow is stationary before executing the next step.

(3) In this step the data stream between the FPGA computers and the cluster nodes is established. This means that the FPGA computers and the cluster nodes are instructed by the Master Computer to go through a handshake procedure after which each cluster node is waiting for data from the corresponding FPGA computer and the FPGA computer is waiting for data from the corresponding camera. The Master Computer waits for confirmation from all devices before executing step four.

\footnotetext{
${ }^{14} \mathrm{In}$ all experiments presented in this thesis the maximum possible propeller acceleration of $2000 \mathrm{rpm} / \mathrm{s}$ is set.

${ }^{15}$ This program was written by Haitao Xu.
} 
(4) Now that the FPGA computers and the cluster nodes are ready for data acquisition, the data transmission of the cameras is started by switching the pre-trigger signal on. After $0.5 \mathrm{~s}$, which is the time between the start of recording and switching on the illumination, step five is executed. Since we measured that the time it takes for two different cameras to process the pre-trigger signal can take up to $0.1 s$, we chose to wait $0.5 s$ to make sure all cameras started recording before the laser is switched on.

(5) As we argued before, the pre-trigger signal is not reliable enough to start all cameras recording at exactly the same time. Therefore, the laser is switched on in this step, $0.5 \mathrm{~s}$ after the recording started, such that the first bright frame can be used for alignment of the image sequences. Then the pre-trigger is switched off again, as the cameras are only sensitive to the rising edges of this signal. Finally a timer for the delay time is started, and after $t_{d e l}$ elapsed, step six is executed.

6 The propeller speed is changed to speed 2 and a timer for the recording time is started and issues the execution of step seven after $t_{r e c}-t_{d e l}$. 
(7) Now that the recording time is elapsed, FSYNC is switched off and therefore the cameras do not send any more images. However, there might still be data in the buffers of the FPGA computers and the cluster nodes. Therefore the Master Computer now waits for those to confirm that the data stream has ended before executing step eight.

(8) After receiving confirmation that the stream stopped, the FPGA computers and the cluster nodes are instructed to disconnect from each other. Moreover the Laser is switched off and the FSYNC is switched on again. If the desired number of movies has been recorded the program ends; otherwise step two is executed and the cycle starts over again.

Note that between steps 4 and 7 , the data stream is sent continuously from the camera to the FPGA computer, and then to the cluster node, where it is received by the server program and written to the HDD. 


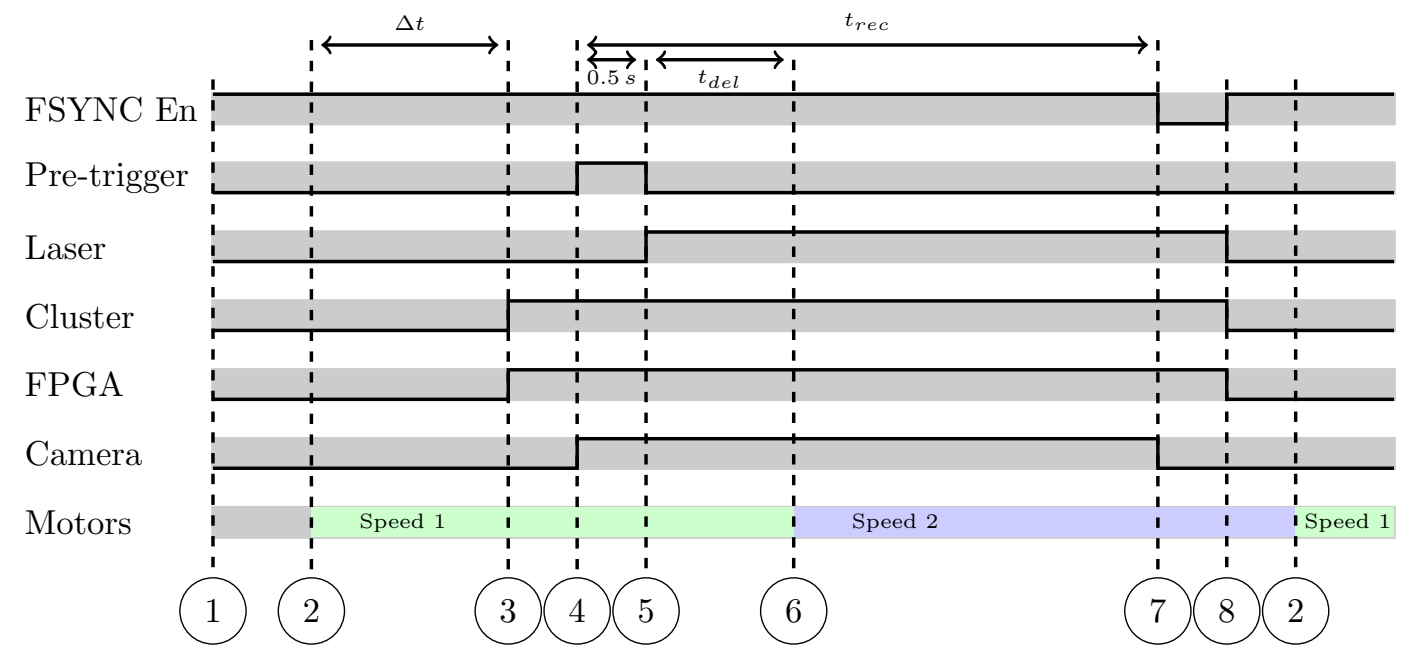

Figure 3.14: Timing diagram of the data acquisition. The dashed vertical lines indicate which instance of the Master Computer program, shown in Fig. 3.15, is effective at that time. Note that the spacing between the vertical lines is not in proportion to the amount of time passed between the corresponding events. Three times are set by the user: $\Delta t$ defines a waiting time between two movies, for the flow to become stationary at speed $1 ; t_{r e c}$ specifies the duration of the recording; $t_{d e l}$ determines when the propeller speed is set to speed 2 after the recording started. The unlabeled times are not specified and depend on the tasks performed in the respective steps and the network communication involved. 


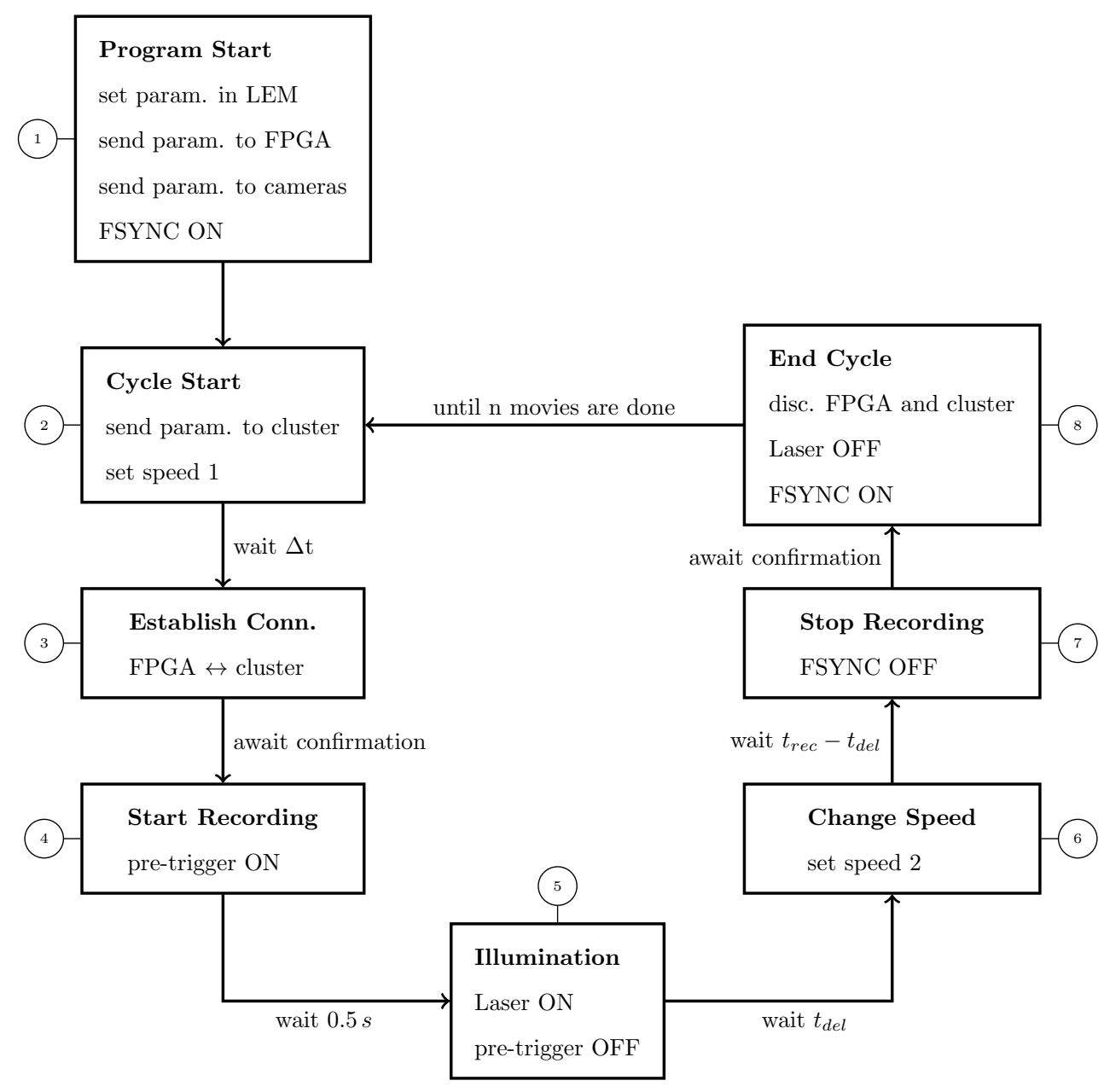

Figure 3.15: Flow chart of the main control program running on the Master Computer. The numbers of the boxes correspond to the timing markers in the timing diagram (Fig. 3.14). Three times are set by the user: $\Delta t$ defines a waiting time between two movies, for the flow to become stationary at speed $1 ; t_{r e c}$ specifies the duration of the recording; $t_{d e l}$ determines when the propeller speed is set to speed 2 after the recording started. 


\section{Chapter 4}

\section{Results}

In this Chapter, the results of the experiments conducted in the scope of this thesis are presented. Our goal was to investigate the response of a turbulent flow to a sudden change of the energy input, which was achieved by a motor-speedup in one case and by switching off the motors in the other case. In order to quantify the transition process between the two energy input states, at first steady state measurements with four different energy input rates were performed and are presented in Section 4.1. The steady state measurements were performed with constant propeller speed at $200 \mathrm{rpm}, 400 \mathrm{rpm}, 300 \mathrm{rpm}$ and $500 \mathrm{rpm}$, for later comparison with the measurements of the transient cases. In Section 4.2 the two cases with a step-function like increase of the large scale forcing, from $200 \mathrm{rpm}$ to $400 \mathrm{rpm}$ and from $300 \mathrm{rpm}$ to $500 \mathrm{rpm}$, are presented. For the investigation of the 
decay of turbulence two more experiments were performed. In both experiments, the energy input to an otherwise stationary forced turbulent flow was stopped by switching off the motors. In one case the initial propeller speed was $200 \mathrm{rpm}$, in the other case $400 \mathrm{rpm}$. The results of these experiments are presented in Section 4.3 .

General parameters, which apply for all data sets presented in this thesis, are listed in Tab. 4.1. The other parameters, that differ from one experiment to another, will be listed in the respective section.

\begin{tabular}{l|c} 
Frame rate & $3 \mathrm{kHz}$ \\
\hline Exposure time & $320 \mu \mathrm{s}$ \\
\hline Frame size & $512 \times 512$ \\
\hline FPGA threshold camera 0 & 150 \\
\hline FPGA threshold camera 1 & 200 \\
\hline FPGA threshold camera 2 & 170 \\
\hline FPGA threshold camera 3 & 170 \\
\hline Tracer particle diameter & $80 \mu \mathrm{m}$ \\
\hline Measurement volume size & $\sim(8 \mathrm{~cm})^{3}$ \\
\hline Spacial resolution & $\sim 160 \mu \mathrm{m} / \mathrm{pixel}$
\end{tabular}

Table 4.1: General parameters, applying to all experiments presented in this thesis. 


\subsection{Steady State}

In this section, the steady state measurements that were acquired with constant propeller speed at $200 \mathrm{rpm}, 400 \mathrm{rpm}, 300 \mathrm{rpm}$ and $500 \mathrm{rpm}$ are presented. For each propeller speed about twelve movies were recorded, each with a duration of 60 s. The Lagrangian Particle Tracking algorithm explained in (Section 3.2) was applied to each recording. In the following, statistical quantities like total kinetic energy $E$, dissipation rate $\epsilon$ etc., that were determined from the four data sets, are presented.

The longitudinal and transverse second order structure functions, $D_{L L}(r)$ and $D_{N N}(r)$, were measured according to Eq. 2.10) and Eq. 2.11), respectively. Ana$\log$ to Eq. 2.10, the longitudinal third order structure function was measured using

$$
D_{L L L}(r)=\left\langle\left[u_{\|}(\boldsymbol{x}+\boldsymbol{r}, t)-u_{\|}(\boldsymbol{x}, t)\right]^{3}\right\rangle .
$$

These three functions were used to determine the energy dissipation rate $\epsilon$. Kolmogorov's theory shows that $\epsilon$ in the inertial range can be represented by $D_{L L}(r)$, $D_{N N}(r)$ and $D_{L L L}(r)$ according to Eq. (2.14), Eq. 2.15) and Eq. (2.25), respectively. Therefore, the functions

$$
\epsilon_{D_{L L}}=\left(\frac{D_{L L}(r)}{C_{2}}\right)^{3 / 2} \frac{1}{r}
$$




$$
\begin{aligned}
\epsilon_{D_{N N}} & =\left(\frac{3}{4} \frac{D_{N N}(r)}{C_{2}}\right)^{3 / 2} \frac{1}{r} \quad \text { and } \\
\epsilon_{D_{L L L}} & =-\frac{5}{4} D_{L L L}(r) \frac{1}{r}
\end{aligned}
$$

are shown in Fig. 4.1, Fig. 4.2, Fig. 4.3 and Fig. 4.4 for the 200 rpm, 300 rpm, $400 \mathrm{rpm}$ and $500 \mathrm{rpm}$ cases, respectively. In consistency with the prediction that $\epsilon$ is constant in the inertial range the data show a plateau in the range between $2-40 \mathrm{~mm}$. However, to minimize the error $\epsilon$ was determined in the range of $4-$ $16 \mathrm{~mm}$ where the three curves show the best agreement. Due to the relatively low particle seeding density in the measurement volume, needed for the LPT algorithm and the real time image compression to work, the probability to observe two tracer particles at a close distance from each other is very low. Therefore, the number of statistics for length scales smaller than $2 \mathrm{~mm}$ is very small and the data show significant scatter.

The total kinetic energy per unit mass of the four steady state experiments was determined by averaging the squares of the measured velocities of all particles found in the measurement volume, $E=\frac{1}{2}\left\langle u^{2}\right\rangle$. From this the root mean square of the velocity fluctuations, $u^{\prime}=\sqrt{2 E / 3}$, was calculated. Here, the factor 3 appears because $u^{\prime}$ is defined for one velocity component and the flow is isotropic. With $u^{\prime}$ and $\epsilon$ other flow parameters can be determined. 


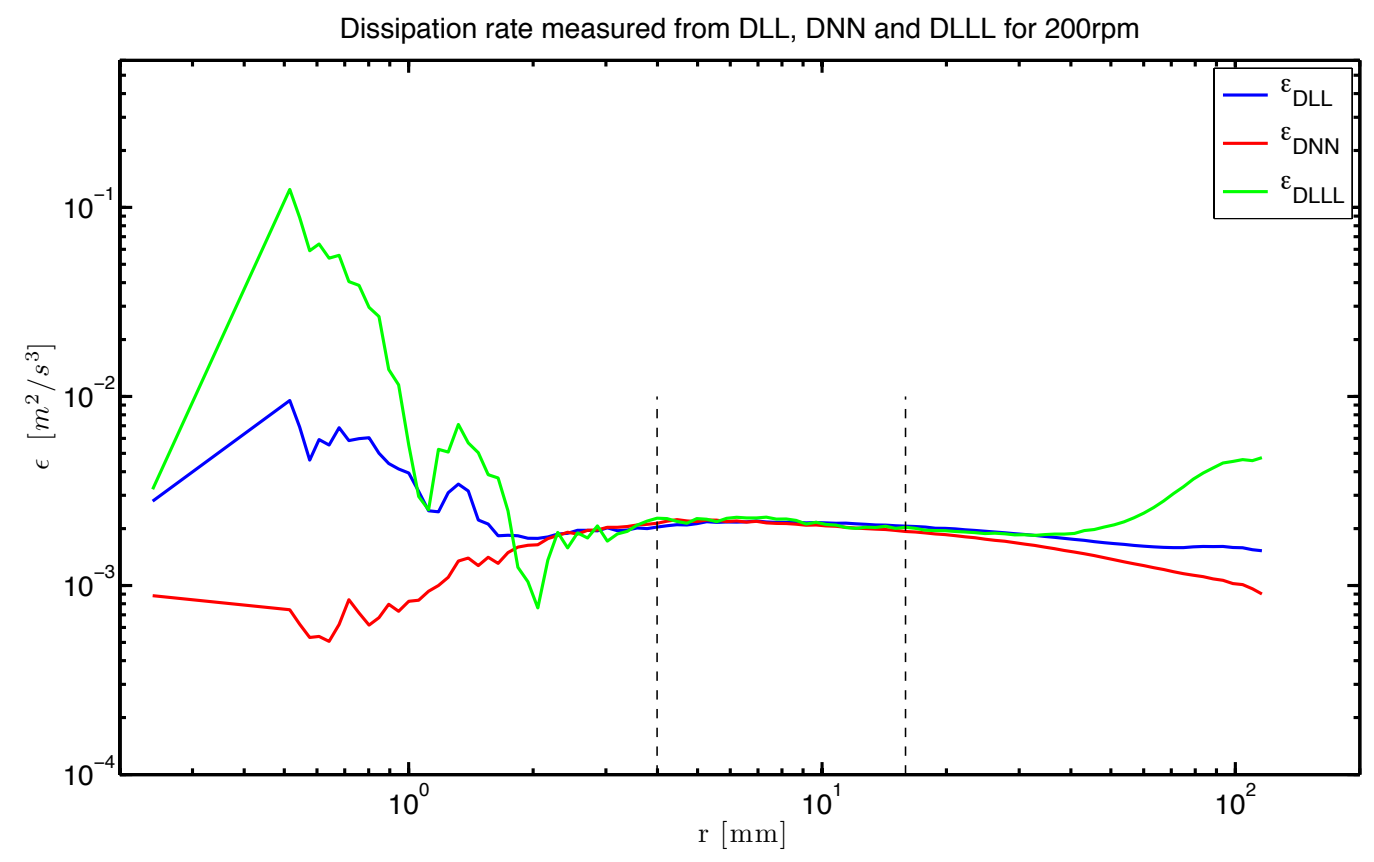

Figure 4.1: Energy dissipation rate $\epsilon$ for steady-state turbulence at a propeller speed of $200 \mathrm{rpm}$, determined from $D_{L L}$ (blue curve), $D_{N N}$ (red curve) and $D_{L L L}$ (green curve) as a function of scale, using Eq. 4.2. The dissipation rate was determined by averaging over all three curves in the range of $4-16 \mathrm{~mm}$ (indicated by the vertical dashed lines) with a value of $\epsilon_{200}=2.1 \cdot 10^{-3} \frac{\mathrm{m}^{2}}{\mathrm{~s}^{3}}$. 


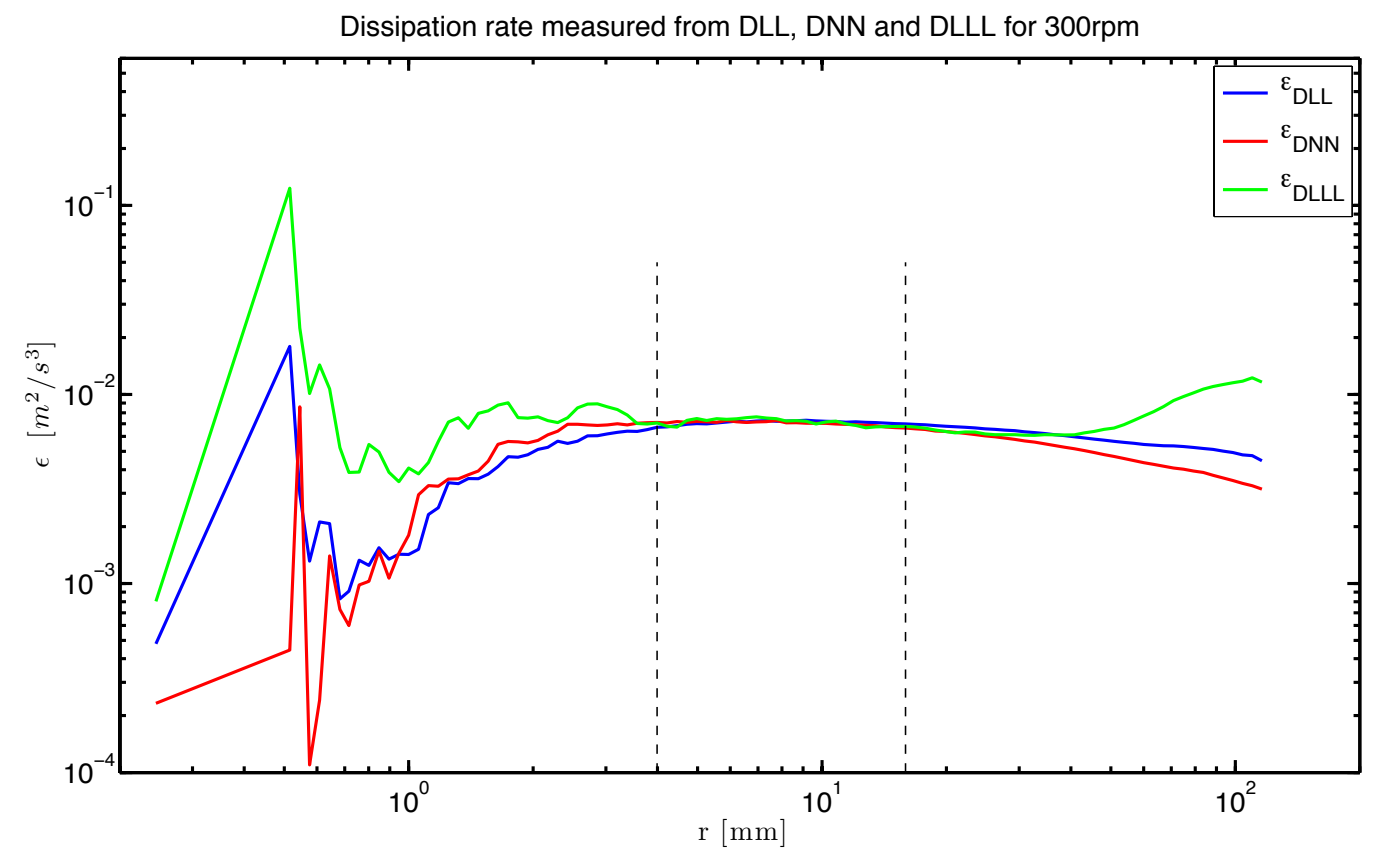

Figure 4.2: Energy dissipation rate $\epsilon$ for steady-state turbulence at a propeller speed of $300 \mathrm{rpm}$, determined from $D_{L L}$ (blue curve), $D_{N N}$ (red curve) and $D_{L L L}$ (green curve) as a function of scale, using Eq. 4.2). The dissipation rate was determined by averaging over all three curves in the range of $4-16 \mathrm{~mm}$ (indicated by the vertical dashed lines) with a value of $\epsilon_{300}=7.1 \cdot 10^{-3} \frac{\mathrm{m}^{2}}{\mathrm{~s}^{3}}$. 


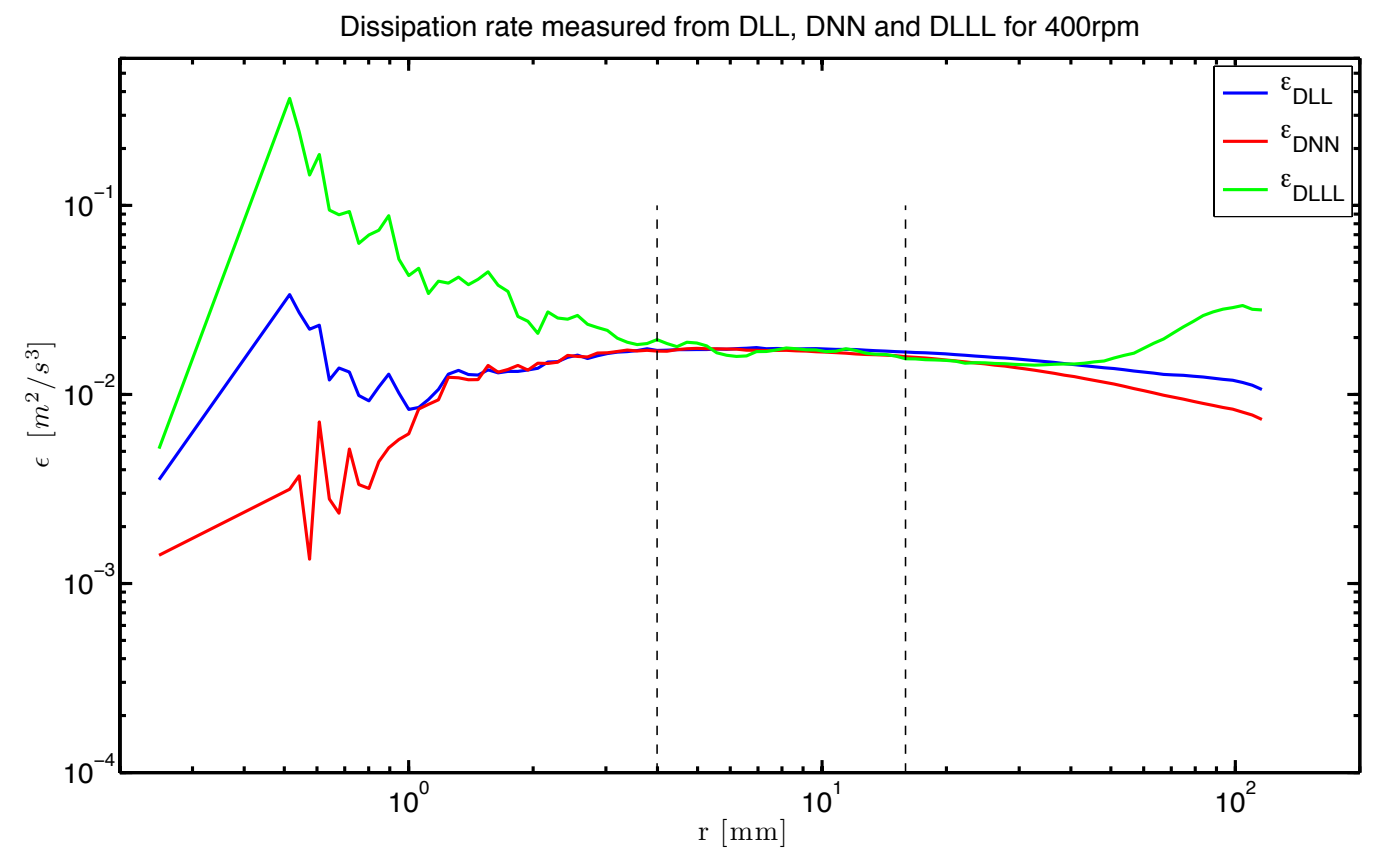

Figure 4.3: Energy dissipation rate $\epsilon$ for steady-state turbulence at a propeller speed of $400 \mathrm{rpm}$, determined from $D_{L L}$ (blue curve), $D_{N N}$ (red curve) and $D_{L L L}$ (green curve) as a function of scale, using Eq. 4.2. The dissipation rate was determined by averaging over all three curves in the range of $4-16 \mathrm{~mm}$ (indicated by the vertical dashed lines) with a value of $\epsilon_{400}=1.7 \cdot 10^{-2} \frac{\mathrm{m}^{2}}{\mathrm{~s}^{3}}$. 


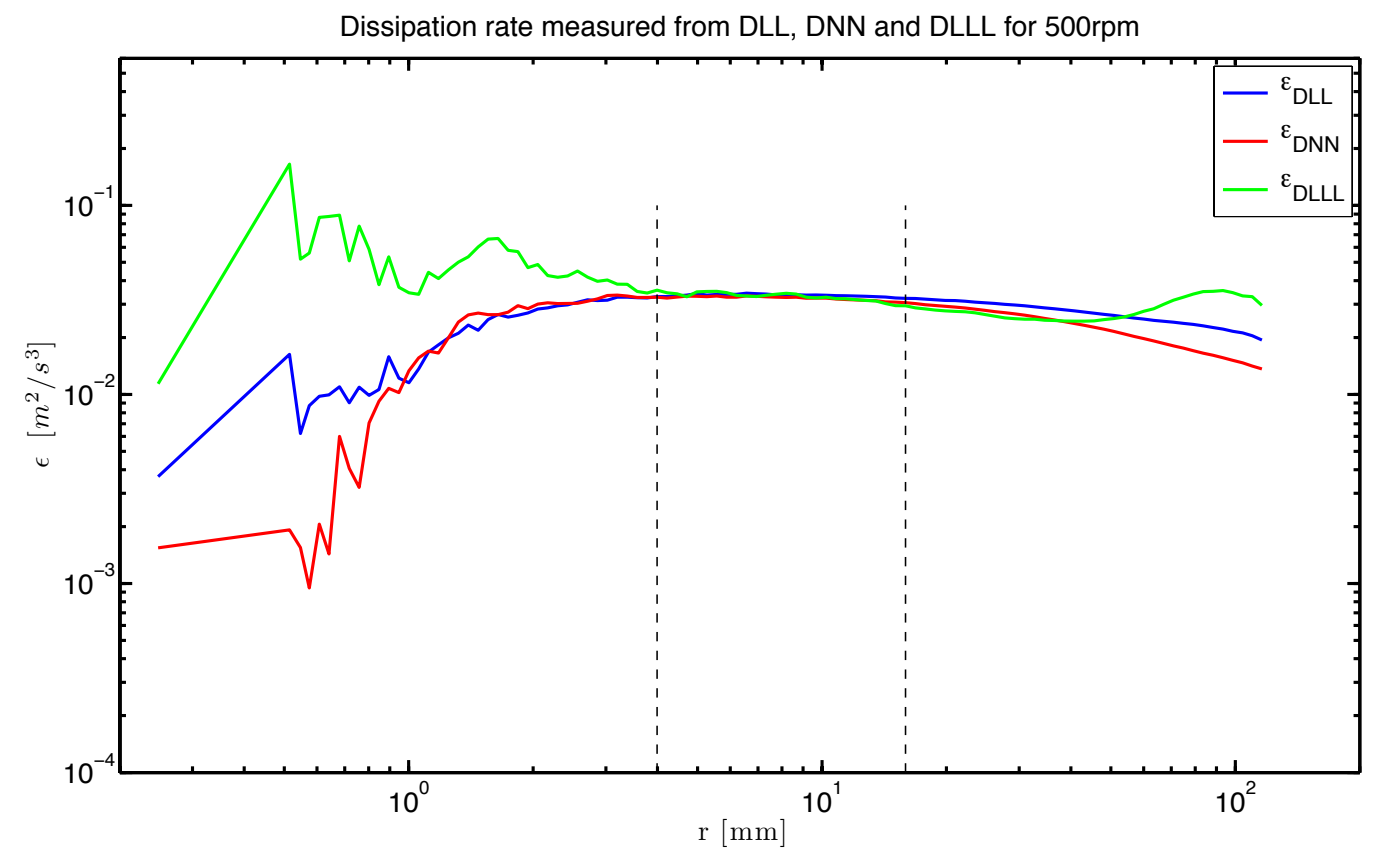

Figure 4.4: Energy dissipation rate $\epsilon$ for steady-state turbulence at a propeller speed of $500 \mathrm{rpm}$, determined from $D_{L L}$ (blue curve), $D_{N N}$ (red curve) and $D_{L L L}$ (green curve) as a function of scale, using Eq. 4.2. The dissipation rate was determined by averaging over all three curves in the range of $4-16 \mathrm{~mm}$ (indicated by the vertical dashed lines) with a value of $\epsilon_{500}=3.3 \cdot 10^{-2} \frac{\mathrm{m}^{2}}{\mathrm{~s}^{3}}$. 
The velocity fluctuation $u^{\prime}$ is a characteristic velocity of the turbulent flow produced in the LEM. Since Eq. 2.7) is valid in the whole inertial range, the forcing length scale, which also characterizes the scale of the largest eddies, can be obtained from $L=u^{\prime 3} / \epsilon$. These quantities could be used to define a Reynolds number $R e=u^{\prime} L / \nu$, but in turbulence research it is more common to define the Reynolds number $R_{\lambda}=u^{\prime} \lambda / \nu$ based on the Taylor micro-scale $\lambda$. For isotropic turbulence $R e$ and $R_{\lambda}$ are related by $R_{\lambda}=\sqrt{15 R e}$ (Pope, 2000). Therefore the Taylor micro-scale based Reynolds number is obtained using $R_{\lambda}=\sqrt{15 u^{\prime 4} /(\epsilon \nu)}$. The Kolmogorov length scale $\eta$ and the Kolmogorov time scale $\tau_{\eta}$ are obtained according to Eq. (2.6) and the large-eddy turnover time is given by $T_{E}=L / u^{\prime}$.

The flow parameters of the steady-state experiments, determined as described above, including the temperature of the working fluid $\theta$ and the corresponding kinematic viscosity $\nu$, are given in Tab. 4.2. Since it was difficult to keep the temperature constant through all experimental realizations, the temperature was different for each data set. Within each data set, however, the temperature was not fluctuating more than $\pm 1^{\circ} \mathrm{C}$. This leaves an error of less than $\sim 3 \%$ on the value for the viscosity and error of less than $\sim 2 \%$ on the quantities derived from that $\left(R_{\lambda}, \eta\right.$ and $\left.\tau_{\eta}\right)$. The error in the velocity measurement was estimated to be $10^{-2} \mathrm{~m} / \mathrm{s}$. The uncertainty in $\epsilon$ was calculated from the standard deviation of the curves to the average. All other errors given in Tab. 4.2 are calculated from those. 


\begin{tabular}{l|c|c|c|c} 
& $200 \mathrm{rpm}$ & $300 \mathrm{rpm}$ & $400 \mathrm{rpm}$ & $500 \mathrm{rpm}$ \\
\hline$E\left[10^{-2} \mathrm{~m}^{2} / \mathrm{s}^{2}\right]$ & $0.5 \pm 0.1$ & $1.0 \pm 0.1$ & $1.8 \pm 0.2$ & $2.7 \pm 0.2$ \\
\hline$u^{\prime}\left[10^{-2} \mathrm{~m} / \mathrm{s}\right]$ & $6 \pm 1$ & $8 \pm 1$ & $11 \pm 1$ & $14 \pm 1$ \\
\hline$\epsilon\left[10^{-2} \mathrm{~m}^{2} / \mathrm{s}^{3}\right]$ & $0.21 \pm 0.01$ & $0.71 \pm 0.03$ & $1.7 \pm 0.1$ & $3.3 \pm 0.2$ \\
\hline$R_{\lambda}$ & $260 \pm 93$ & $310 \pm 75$ & $350 \pm 64$ & $390 \pm 58$ \\
\hline$L[\mathrm{~mm}]$ & $87 \pm 47$ & $84 \pm 30$ & $80 \pm 22$ & $80 \pm 18$ \\
\hline$\eta[\mu \mathrm{m}]$ & $157 \pm 2$ & $115 \pm 1$ & $93 \pm 1$ & $79 \pm 1$ \\
\hline$\tau_{\eta}[\mathrm{ms}]$ & $22.6 \pm 0.5$ & $12.3 \pm 0.3$ & $8.0 \pm 0.2$ & $5.7 \pm 0.1$ \\
\hline$T_{E}[\mathrm{~s}]$ & $1.5 \pm 0.6$ & $1.0 \pm 0.2$ & $0.7 \pm 0.1$ & $0.58 \pm 0.09$ \\
\hline$\theta\left[{ }^{\circ} \mathrm{C}\right]$ & $16.6 \pm 1$ & $17.0 \pm 1$ & $16.6 \pm 1$ & $16.9 \pm 1$ \\
\hline$\nu\left[10^{-6} \mathrm{~m}^{2} / \mathrm{s}\right]$ & $1.09 \pm 0.03$ & $1.08 \pm 0.03$ & $1.09 \pm 0.03$ & $1.08 \pm 0.03$
\end{tabular}

Table 4.2: Flow parameters for the steady-state experiments with propeller speeds $200 \mathrm{rpm}, 300 \mathrm{rpm}, 400 \mathrm{rpm}$ and $500 \mathrm{rpm}$. 


\subsection{Step-up of Energy Injection}

In this set of experiments, the response of the turbulent flow in the LEM to a stepfunction like increase of the large-scale forcing was investigated. The timing cycle for the $300 \mathrm{rpm}$ to $500 \mathrm{rpm}$ data set was set such that all propellers were initially set to $300 \mathrm{rpm}$ for about $2.5 \mathrm{~min}\left(150 T_{E}\right)$ to make sure that the flow was statistically stationary. Then the LPT measurement started and the propeller speed remained at $300 \mathrm{rpm}$ for another $5 \mathrm{~s}$ before it was increased to $500 \mathrm{rpm}$. The energy input stayed on the high level for $60 \mathrm{~s}$. Then the data acquisition was terminated and the propeller speed again reduced to $300 \mathrm{rpm}$ for $2.5 \mathrm{~min}$ before the next recording started. That means, videos with a duration of $65 s$ were recorded, where the first $5 s$ were recorded with a low energy input. In total, 326 videos were recorded for the $300 \mathrm{rpm}-500 \mathrm{rpm}$ case.

For the $200 \mathrm{rpm}$ to $400 \mathrm{rpm}$ experiment the waiting time for the flow to become stationary between two recordings was increased to $5 \mathrm{~min}\left(200 T_{E}\right)$ to account for the longer large-eddy turnover time at the lower motor speed and the higher energy injection rate. The recording time was reduced to $45 s$ as the measurements of the $300 \mathrm{rpm}$ - $500 \mathrm{rpm}$ case suggested that this recording time should be sufficient. Again the first $5 s$ were recorded with low energy input and the rest with high energy input. In total, 314 recordings were acquired for this data set.

To obtain time dependent statistics, the recordings of each data set were phase 
averaged. In order to do that, the moment of motor-speed-up in each video was determined. As described in Section 3.1, when the motors sped up a laser pointer produced a bright spot on the sensor of one of the cameras. By finding the increase of intensity in the respective region of the image sequences of that camera the moment of motor-speed-up was determined relative to the moment when the measurement volume illumination (Nd:YAG laser) was switched on. Fig. 4.5 shows that the time of the motor-speed-up can vary up to about $0.2 \mathrm{~s}$ from movie to movie, which was a result of imprecision of the software timers used in the control program and latencies in the communication of the involved devices through Ethernet and CAN bus.

After determining the moment of motor-speed-up each recording was divided into bins with a duration of $0.1 \mathrm{~s}$, with the motor-speed-up being defined as $t=0$. Then the data from the respective bins of all recordings were averaged to obtain time dependent statistics.

At first it had to be confirmed, that the turbulent flows of both cases, $200 \mathrm{rpm}$ to $400 \mathrm{rpm}$ and $300 \mathrm{rpm}$ to $500 \mathrm{rpm}$ were steady at the low energy input when the recordings started and reached the steady state corresponding to the high energy input during the recording. In order to do that, the statistics of the first $5 s$ of the datasets were averaged to compare to the $200 \mathrm{rpm}$ and $300 \mathrm{rpm}$ steady state cases, and the statistics from $20 \mathrm{~s}$ to the end of the recordings were averaged to 


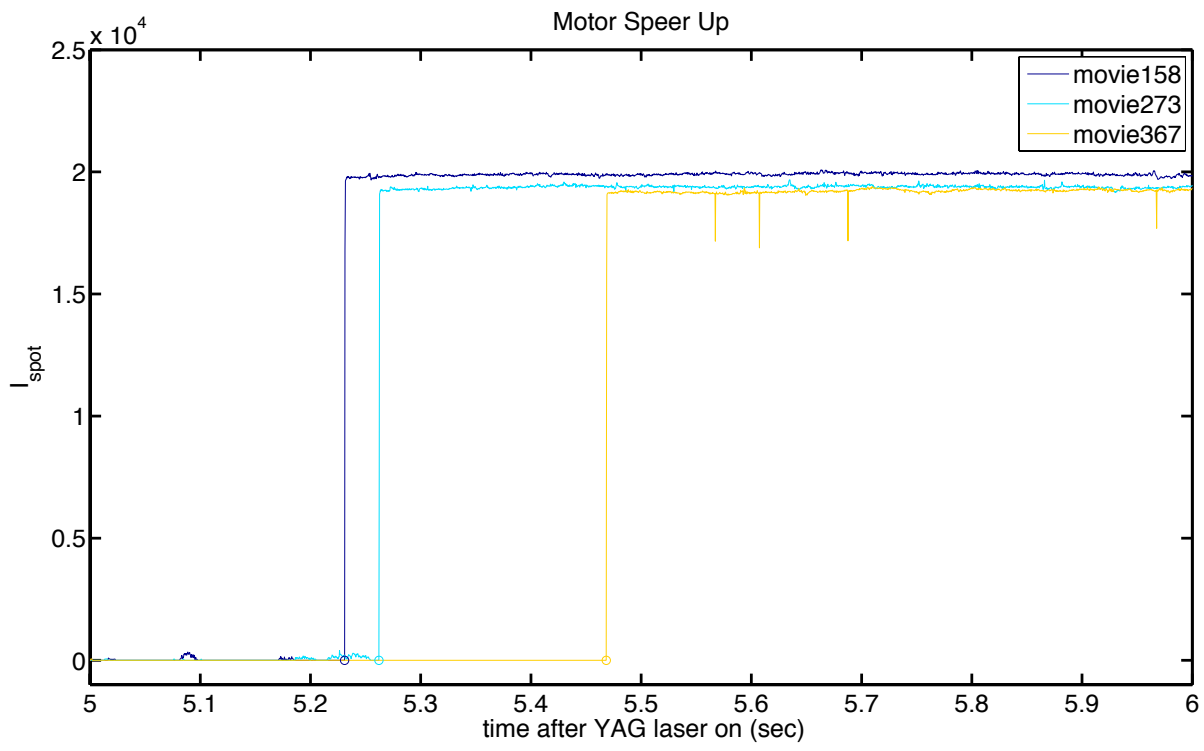

Figure 4.5: Intensity in a 20 by 20 pixel region of the camera sensor that captures the laser pointer lighting up to determine the moment of the motor-speed-up. As example, the signal of three movies of the $200 \mathrm{rpm}$ to $400 \mathrm{rpm}$ data set is shown. On average the motors speeded up at $5.26 \mathrm{~s}$ after the Nd:YAG laser was switched on, like in movie 273. Movies 158 and 367 represent extreme cases.

compare to the $400 \mathrm{rpm}$ and $500 \mathrm{rpm}$ steady state cases. The data was analyzed as explained in Section 4.1 and the flow parameters obtained that way are presented in Tab. 4.3 and compare very well to the parameters of the steady states, listed in Tab. 4.2. This confirms that the turbulent flows produced for this two data sets went through the full transition from a steady state at a low energy input to a 
steady state at a higher energy input.

Now the temporal evolution of the flow from one steady state to the other shall be examined. We want to start with the kinetic energy $E(t)$, which is expected to equal the kinetic energy of the low energy input case, $\left\langle E_{L}\right\rangle$, before the motors change speed $(t \leq 0)$. After sufficiently long time $E(t)$ is then expected to reach the kinetic energy of the high energy input case $\left\langle E_{H}\right\rangle$. Therefore, in Fig. 4.6 the quantity $\left(E(t)-\left\langle E_{L}\right\rangle\right) /\left(\left\langle E_{H}\right\rangle-\left\langle E_{L}\right\rangle\right)$ is shown as a function of time for both step-up data sets, where time is in units of the large eddy turnover time of the final stationary state, $T_{E}$, respectively. Both data sets show that there is a time delay of about $2 T_{E}$ between the motor-speed-up and the moment when the kinetic energy in the observation volume starts to rise. This is most likely due to the fact that the momentum, generated at the propellers, needs time to be transported from the propellers to the measurement volume with a distance of about $D=20 \mathrm{~cm}$ in between. The turbulence transport takes time of the order of $D / u^{\prime}=(D / L) \cdot\left(L / u^{\prime}\right)=(D / L) \cdot T_{E}$. In our experiments the integral scale $L$ is nearly constant over the range of $R_{\lambda}$ explored (Tab. 4.3). Therefore, the observed delay time is approximately $2 T_{E}$, independent of $R_{\lambda}$. This delay has also been observed in experiments in a von Kármán flow (the so-called French Washing Mashine) with sinusoidal forcing (Cadot et al., 2003).

The $300 \mathrm{rpm}$ to $500 \mathrm{rpm}$ data set (red curve) shows fairly strong fluctuations 


\begin{tabular}{l|c|c|c|c} 
& $\begin{array}{c}200 \mathrm{rpm} \\
\text { from } \\
-5-0 \mathrm{~s} \text { of } \\
\end{array}$ & $\begin{array}{c}300 \mathrm{rpm} \\
\text { from } \\
-5-0 \mathrm{~s} \text { of } \\
200-400 \mathrm{rpm}\end{array}$ & $\begin{array}{c}400 \mathrm{rpm} \\
\text { from }\end{array}$ & $\begin{array}{c}500 \mathrm{rpm} \\
\text { from } \\
\end{array}$ \\
\hline$\langle E\rangle\left[10^{-2} \mathrm{~m}^{2} / \mathrm{s}^{2}\right]$ & $0.5 \pm 0.1$ & $1.1 \pm 0.1$ & $1.8 \pm 0.2$ & $2.7 \pm 0.2$ \\
\hline$u^{\prime}\left[10^{-2} \mathrm{~m} / \mathrm{s}\right]$ & $5.6 \pm 1$ & $8.4 \pm 1$ & $11.1 \pm 1$ & $13.8 \pm 1$ \\
\hline$\epsilon\left[10^{-2} \mathrm{~m}^{2} / \mathrm{s}^{3}\right]$ & $0.20 \pm 0.01$ & $0.72 \pm 0.03$ & $1.7 \pm 0.1$ & $3.4 \pm 0.2$ \\
\hline$R_{\lambda}$ & $250 \pm 91$ & $310 \pm 73$ & $350 \pm 63$ & $380 \pm 56$ \\
\hline$L[\mathrm{~mm}]$ & $87 \pm 47$ & $83 \pm 30$ & $81 \pm 22$ & $78 \pm 17$ \\
\hline$\eta[\mu \mathrm{m}]$ & $163 \pm 2$ & $116 \pm 1$ & $95 \pm 1$ & $79 \pm 1$ \\
\hline$\tau_{\eta}[\mathrm{ms}]$ & $23.7 \pm 0.6$ & $12.3 \pm 0.2$ & $8.0 \pm 0.2$ & $5.7 \pm 0.1$ \\
\hline$T_{E}[\mathrm{~s}]$ & $1.6 \pm 0.6$ & $1.0 \pm 0.2$ & $0.7 \pm 0.1$ & $0.56 \pm 0.09$ \\
\hline$\theta\left[{ }^{\circ} \mathrm{C}\right]$ & $15.6 \pm 1$ & $16.2 \pm 1$ & $15.6 \pm 1$ & $16.2 \pm 1$ \\
\hline$\nu\left[10^{-6} \mathrm{~m}^{2} / \mathrm{s}\right]$ & $1.12 \pm 0.03$ & $1.10 \pm 0.03$ & $1.12 \pm 0.03$ & $1.10 \pm 0.03$ \\
\hline
\end{tabular}

Table 4.3: Flow parameters of the steady phases of the $200 \mathrm{rpm}$ to $400 \mathrm{rpm}$ and the $300 \mathrm{rpm}$ to $500 \mathrm{rpm}$ experiments. The parameters were obtained by averaging the time dependent statistics over $5 s$ before the propeller speed change and from $20 s$ after the propeller speed change to the end of the recording. The time of the speed change was defined to be $t=0 \mathrm{~s}$. 
around the low energy value and appears to be steady again at $20 T_{E}$ after the change of motor speed. To avoid a possible effect of the previous high energy state on the initial low energy state of the new recording, the waiting time between two recordings was increased, and the recording time was decreased for the $200 \mathrm{rpm}$ to $400 \mathrm{rpm}$ data set (blue curve). This data set shows smaller fluctuations before the motor speed change and reaches the high energy steady state after about $25 T_{E}$.

Fig. 4.6 shows that the turbulent flows in both experiments adjusted to the new energy input within the time of the observation. From the energy cascade picture one would expect different scales to adjust to the new energy input at different times. The forcing is performed at the large scales and the energy needs time to propagate down the cascade to the small scales. Therefore, the energy content of the large scales should increase earlier, compared to the energy content of smaller scales. As an indicator for the energy content at a given scale the velocity structure functions were used.

In Fig. 4.7, the evolution of $D_{L L}(r, t)$ of the $200 \mathrm{rpm}$ to $400 \mathrm{rpm}$ dataset for different scales is shown. The curve is normalized by $D_{L L}(r)$ of the high energy input case. The curves for all scales rise to the higher level together without showing any scale being particularly faster than any other. In Fig. 4.8, the same quantity is shown as a function of scale at different times after the speed-up. The vertical dashed line marks the large scale $L \sim 900 r / \eta$. Data points in the 


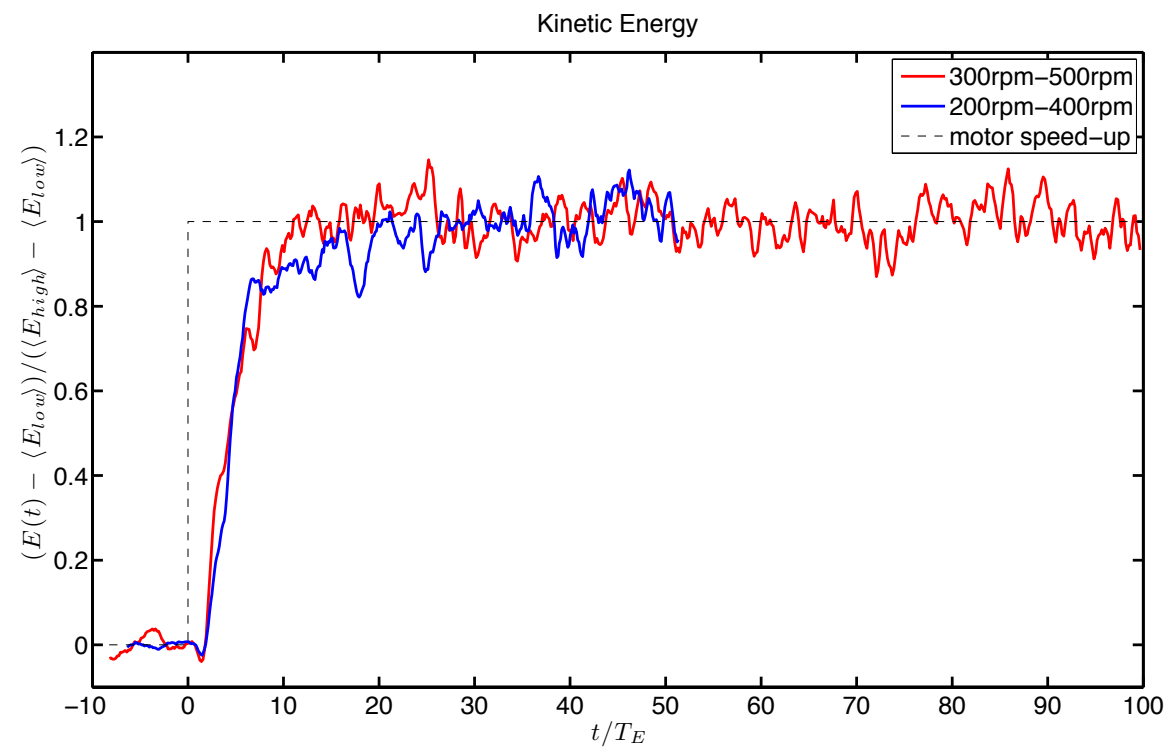

Figure 4.6: Normalized kinetic energy $\left(E(t)-\left\langle E_{L}\right\rangle\right) /\left(\left\langle E_{H}\right\rangle-\left\langle E_{L}\right\rangle\right)$ as a function of large-eddy turnover time. The red curve shows the $300 \mathrm{rpm}$ to $500 \mathrm{rpm}$ and the blue curve shows the $200 \mathrm{rpm}$ to $400 \mathrm{rpm}$ dataset. The kinetic energy $E(t)$ of the step up experiments is normalized with the kinetic energies of the respective steady state energies for high and low energy input, $\left\langle E_{H}\right\rangle$ and $\left\langle E_{L}\right\rangle$, respectively. Time was non-dimensionalized with the large-eddy turnover time $T_{E}$ of the respective high energy state. The values for $\left\langle E_{H}\right\rangle,\left\langle E_{L}\right\rangle$ and $T_{E}$ are listed in Tab. 4.3. The dashed line indicates when the motors speed up. 


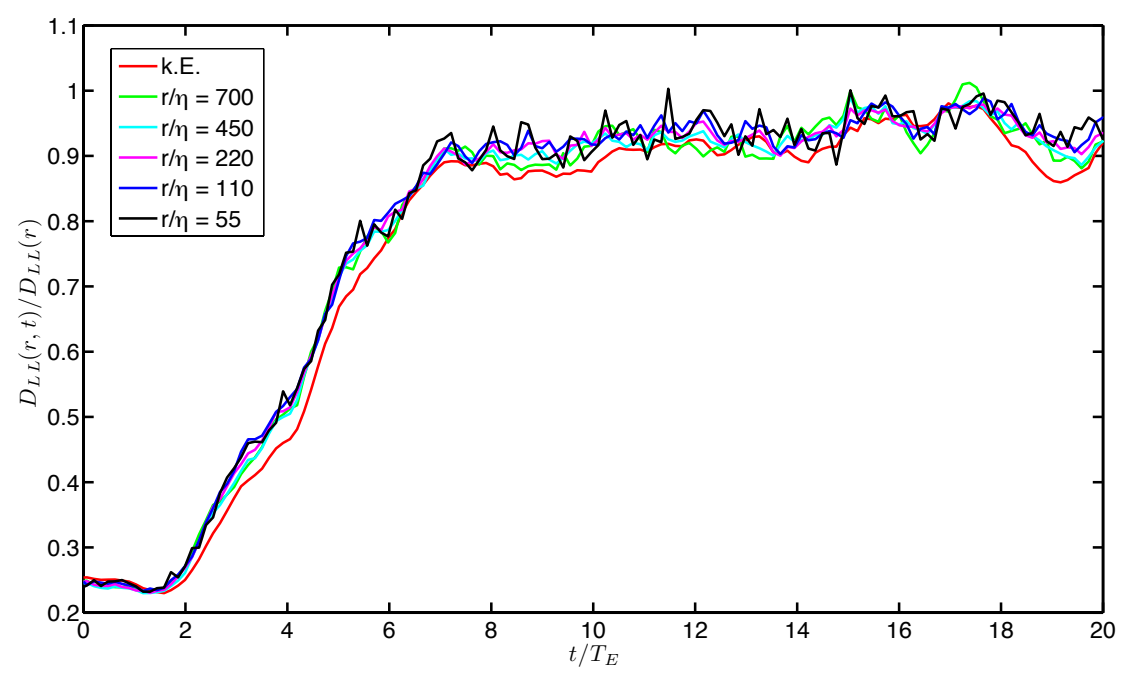

Figure 4.7: $D_{L L}(r, t)$, normalized by $D_{L L}(r)$ of the high energy input case, as a function of large eddy turnover time after motor-speed-up. The curve is shown for different values of $r / \eta$ together with the kinetic energy for comparison. The measurements are taken from the $200 \mathrm{rpm}$ to $400 \mathrm{rpm}$ dataset.

scale range larger than that are more likely to be influenced by the particular design of the experiment as they are outside of the inertial range. Moreover, the measurement volume is not spherical beyond this scale and velocities found with that distances might have a preferential direction. The scales in the range smaller than $L \sim 900 r / \eta$ all seem to adjust to the new forcing at about the same rate.

The full information of $D_{L L}(r, t)$, normalized by $D_{L L}(r)$ of the high energy input case, as a function of scale and time is given in Fig. 4.9 where the large scatter at 


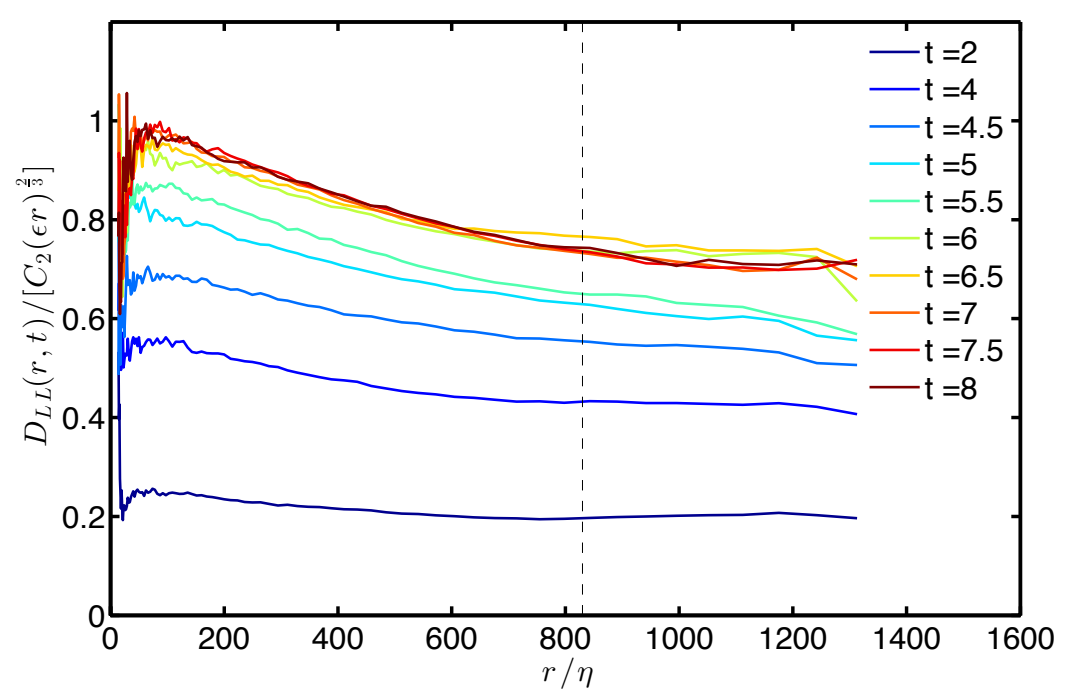

Figure 4.8: $D_{L L}(r, t)$, compensated by the inertial range scaling $C_{2}(\epsilon r)^{2 / 3}$, as a function of $r / \eta$. The curves for different values of time after motor-speed-up are shown. The vertical dashed line marks $900 \mathrm{r} / \eta$, the size of the large scale $L=u^{\prime 3} / \epsilon$. The measurements are taken from the $200 \mathrm{rpm}$ to $400 \mathrm{rpm}$ dataset.

small scales is due to unconverged statistics and limited spatial resolution. Like Fig. 4.7 and Fig. 4.8 , it shows that all scales follow the change of energy input at about the same rate.

From Fig. 4.9 it can be seen that at later times $\left(t / T_{E} \geq 20\right)$, when the flow is already steady at the high energy input, the averaged structure functions still fluctuate significantly even after averaging over 300 realizations. Furthermore, Fig. 4.10 shows the kinetic energy of several individual realizations in comparison 


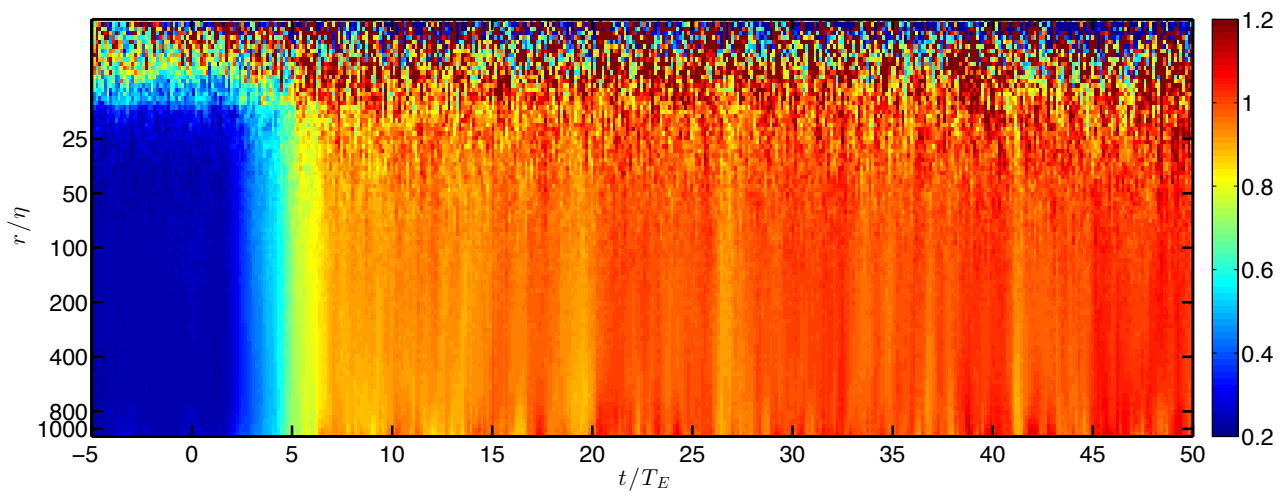

Figure 4.9: Plot of $D_{L L}(r, t)$ (color coded), normalized by $D_{L L}(r)$ of the low energy input case, as a function of scale and time.

with the kinetic energy averaged over all recordings shown in Fig. 4.6. The plot shows that the fluctuations occurring in the single recordings are very strong and, that not all realizations seem to adapt to the new energy input at the same time. When considering the moment at which the fluctuations of the kinetic energy rise above unity for the first time as the time when the flow in the measurement volume starts to adapt to the change in motor speed, it can clearly be seen that this happens at very different times. This is due to the fact that the time it takes for the agitated fluid at the propellers to be convected to the measurement volume is a fluctuating quantity, despite that its average value is approximately $2 T_{E}$. This therefore raises the question whether the way time $t=0$ for the phase averaging was determined (by finding the moment of motor-speed-up) is suitable to observe 


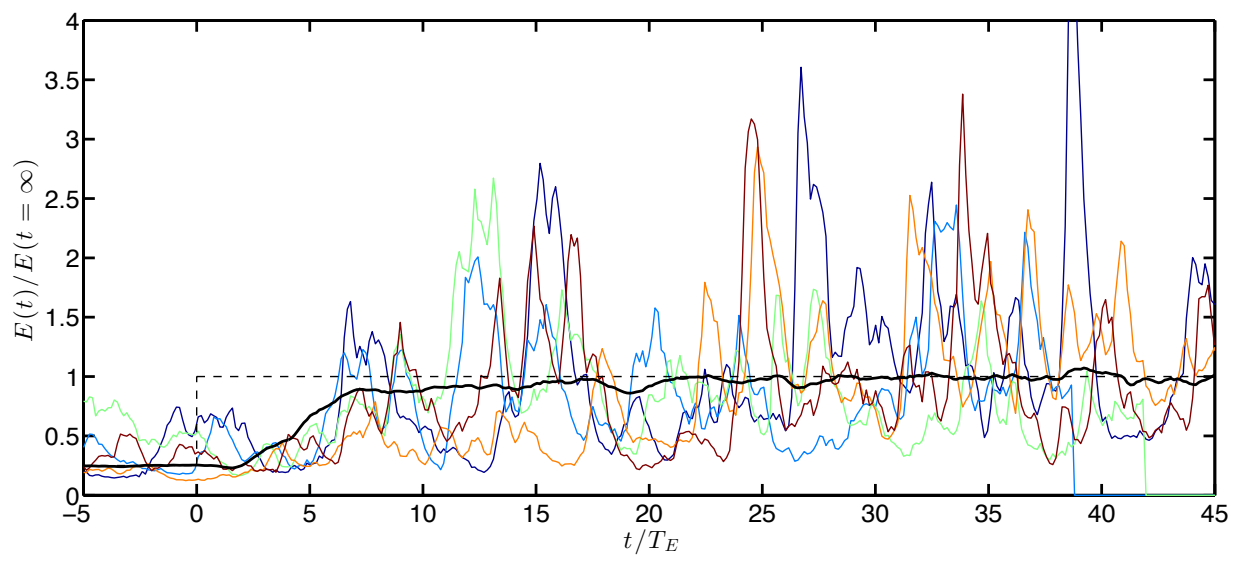

Figure 4.10: Normalized kinetic energy as a function of time. The black curve shows the average over all realizations of the $200 \mathrm{rpm}$ to $400 \mathrm{rpm}$ dataset while the other curves show single realizations.

the response of different scales at different times or if the effect was averaged out.

Since the scale-response that we are interested in should, in principle, also be present in the fluctuations in the steady part of the recordings, we therefore investigated these fluctuations in detail. At first, the events of strong fluctuations were identified by selecting local maxima in kinetic energy that were above $2 E_{H}$. All maxima from all realizations that occurred in the steady part (after $20 T_{E}$ ) were considered. Then a phase averaging was performed with those events including $2 T_{E}$ before and after the maxima and by defining the peak of the kinetic energy to be $t=0$ for the individual events. The structure functions, $D_{L L}(r, t)$ obtained 


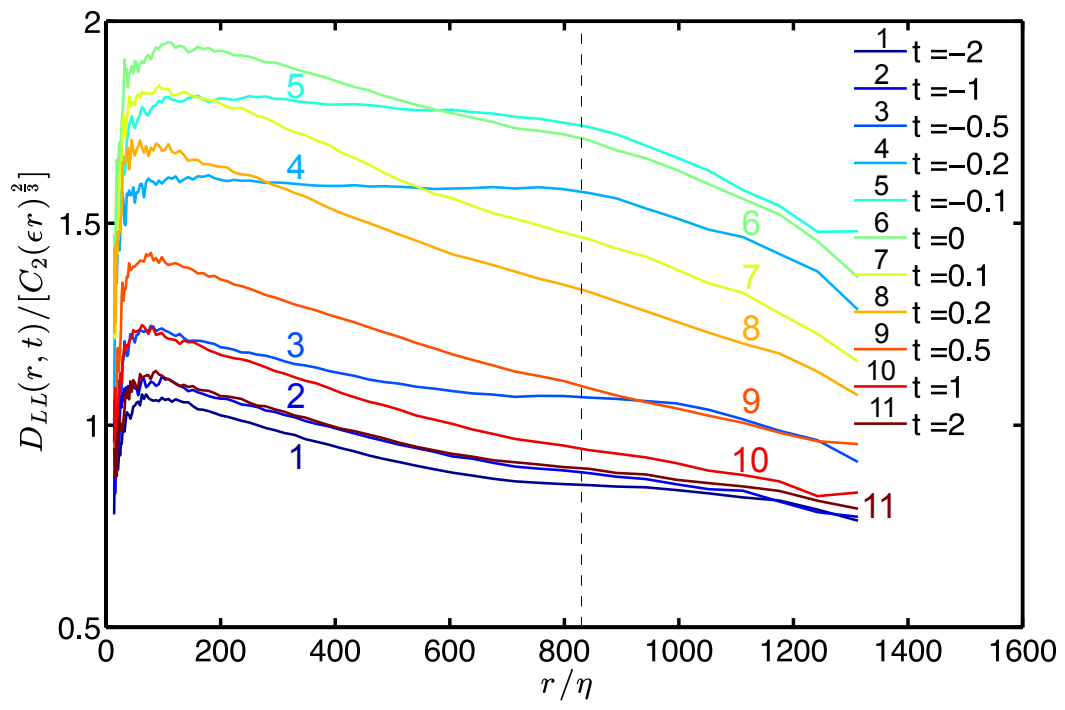

Figure 4.11: Responses of $D_{L L}(r, t)$ to large surpluses of kinetic energy at $t=0$. In the plot, several curves of $D_{L L}(r, t)$ compensated by the inertial range scaling $C_{2}(\epsilon r)^{2 / 3}$, are shown as a function of $r / \eta$, where $\epsilon$ is the energy dissipation rate at the steady state. The vertical dashed line marks $900 r / \eta$, the size of the large scale $L=u^{\prime 3} / \epsilon$. The measurements are taken from the $200 \mathrm{rpm}$ to $400 \mathrm{rpm}$ dataset in the statistically steady regime from $t / T_{E}=20$ to the end of the recording.

in this way then provide information on the scale-dependent response to large surpluses in kinetic energy.

Fig. 4.11 shows $D_{L L}(r, t)$ thus obtained as function of $r / \eta$, compensated by the K41 type inertial range scaling, $C_{2}(\epsilon r)^{2 / 3}$, where $\epsilon$ is the energy dissipation rate at the steady state. The curves corresponding to several different times before and 
after the peak of the kinetic energy are shown. It can be seen that the large scales rise earlier than the small scales. Especially for $-0.5 \leq t / T_{E} \leq-0.1$, the large scales increase much faster than the small scales, which leads to the change in the shape of the compensated $D_{L L}(r, t)$. After approximately $t=0$, the smaller scales rise a bit further to reach the peak of the fluctuation. Then all scales drop back to the steady state level.

This delay of response in the small scales can be better seen in Fig. 4.12, in which $D_{L L}(r, t)$ for several scales $r / \eta$, normalized by the corresponding values of $D_{L L}(r)$ at the steady state are shown as a function of time. Different curves for different scales in the inertial range are shown together with the change of kinetic energy for reference. This plot clearly shows that after the increase of kinetic energy, the large scales increase first. The smaller scales then follow at a later time.

In Fig. $4.13, D_{L L}(r, t)$ in response to a surplus in kinetic energy, normalized by $D_{L L}(r)$ at the steady state, are shown as a function of both scale and time. In this figure, the color-coding is such that the blue color means that $D_{L L}(r, t)$ is close to the steady-state value, while the red color represents large surges compared to the steady-state value. It is clear that the surges in the large scales appear first, then propagate down to smaller scales. This propagation might be interpreted as the energy cascade through scales. According to K41, the cascade time is 


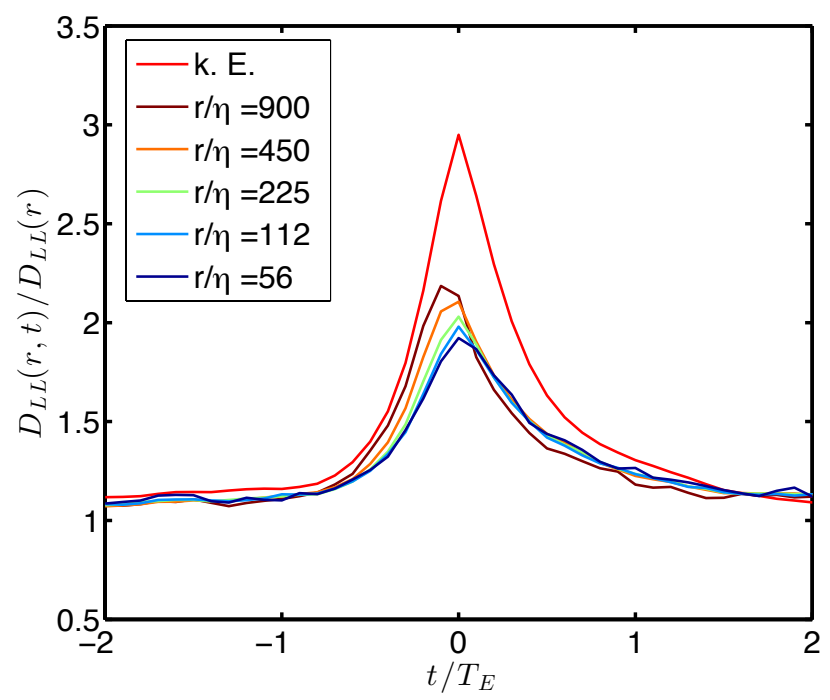

Figure 4.12: The change of $D_{L L}(r, t)$ in response to large surpluses in kinetic energy, normalized by $D_{L L}(r)$ at the steady state, as a function of time. The curves for different values of $r / \eta$ are shown together with the kinetic energy for comparison. The measurements are taken from the $200 \mathrm{rpm}$ to $400 \mathrm{rpm}$ dataset in the statistically steady regime, i.e. from $t / T_{E}=20$ to the end of the recording.

$\tau(r) \sim\left(r^{2} / \epsilon\right)^{1 / 3}$. The dashed line in Fig. 4.13 shows such a relation, which fits reasonably well with the observation. From such a model, the cascade time from large to small scales can be estimated to be $0.2-0.3 T_{E}$, which is in agreement with previous observations from direct numerical simulation (DNS) data (Pumir, 1996; Pearson et al., 2004). 


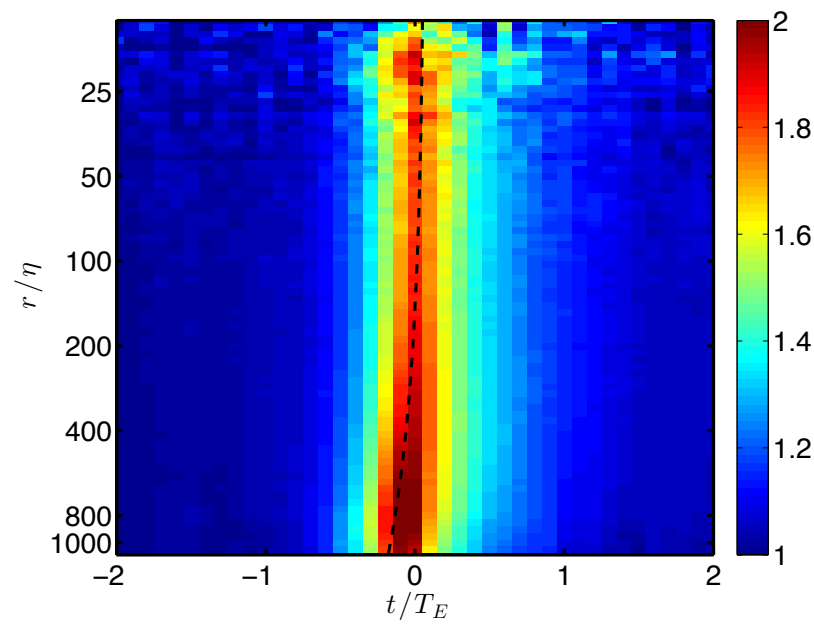

Figure 4.13: Color coding of $D_{L L}(r, t)$ in response to a surplus in kinetic energy, normalized by $D_{L L}(r)$ at the steady state, as a function of both scale and time. The dashed line shows a delay in response according to K41 scaling: $\tau(r) \sim r^{2 / 3}$.

\subsection{Decay of Turbulence}

A natural counterpart to the step increase in energy injection that we studies in the previous section is the step decrease in energy injection. In particular, when the energy injection is completely turned off, the corresponding turbulence decay is a classical problem in turbulence research and can be dated back to the beginning of modern fluid mechanics (Taylor, 1935; Kolmogorov, 1941a). Traditionally, the decay was studied in turbulent flows behind a grid in a wind tunnel. The length of the tunnel, however, limited the duration of the decay that can be observed. In 
out experiments, we are not limited by such a constraint and can observe for much longer times compared to previous work.

Two data sets, about 130 movies each, were recorded to study the decay of turbulence. In one case the turbulence was initially at the steady state maintained with a propeller speed of $200 \mathrm{rpm}$, in the other case with an initial propeller speed of $400 \mathrm{rpm}$. In both cases, recordings with a duration of $65 \mathrm{~s}{ }^{1}$ were realized. The propellers were turned off $5 s$ after the start of the recording. Between two recordings, the flow was forced for 5 min with the respective propeller speed to ensure stationarity before the measurement started.

The time dependent statistics were obtained as explained in Section 4.2 , with the moment of propeller shut-off being defined as $t=0$. By averaging over the first $5 s$ of all realizations of the two data sets, the flow parameters of the steady state of the flow, before the motors were switched off, were determined (Tab. 4.4). They agree well with the corresponding parameters of the separate steady state experiments (Tab. 4.2).

Fig. 4.14 shows the kinetic energy of the two experiments as a function of time. Only data after the stopping of the motors was plotted and it can be seen that the kinetic energy in the measurement volume stays constant for about $1 T_{E}$ before it starts to decay, which is comparable to the response delay observed in the step-up

\footnotetext{
${ }^{1}$ corresponding to $43 T_{E}$ at $200 \mathrm{rpm}$ and $92 T_{E}$ at $400 \mathrm{rpm}$
} 


\begin{tabular}{l|c|c} 
& $\begin{array}{c}200 \mathrm{rpm} \\
\text { measured from } \\
-5-0 \mathrm{~s} \text { of } \\
200-0 \mathrm{rpm}\end{array}$ & $\begin{array}{c}400 \mathrm{rpm} \\
\text { measured from } \\
-5-0 \mathrm{~s} \text { of } \\
400-0 \mathrm{rpm}\end{array}$ \\
\hline$\langle E\rangle\left[10^{-2} \mathrm{~m}^{2} / \mathrm{s}^{2}\right]$ & 0.45 & 1.85 \\
\hline$u^{\prime}\left[10^{-2} \mathrm{~m} / \mathrm{s}\right]$ & 5.52 & 11.06 \\
\hline$\epsilon\left[10^{-2} \mathrm{~m}^{2} / \mathrm{s}^{3}\right]$ & 0.20 & 1.75 \\
\hline$R_{\lambda}$ & 270 & 381 \\
\hline$L[\mathrm{~mm}]$ & 83.2 & 77.1 \\
\hline$\eta[\mu \mathrm{m}]$ & 136 & 79 \\
\hline$\tau_{\eta}[\mathrm{ms}]$ & 20.9 & 7.1 \\
\hline$T_{E}[\mathrm{~s}]$ & 1.51 & 0.70 \\
\hline$\theta\left[{ }^{\circ} \mathrm{C}\right]$ & 25.4 & 25.7 \\
\hline$\nu\left[10^{-6} \mathrm{~m}^{2} / \mathrm{s}\right]$ & 0.88 & 0.88
\end{tabular}

Table 4.4: Flow parameters of the steady phases of the decay experiments started from propeller speed of $200 \mathrm{rpm}$ and $400 \mathrm{rpm}$, respectively. The parameters were obtained by averaging the statistics over $5 s$ before the propellers were stopped. The time of the stopping of the propellers was defined to be $t=0 \mathrm{~s}$. These parameters are in good agreement with the corresponding parameters of the separate steady state experiments at the same speeds shown in (Tab. 4.2). 


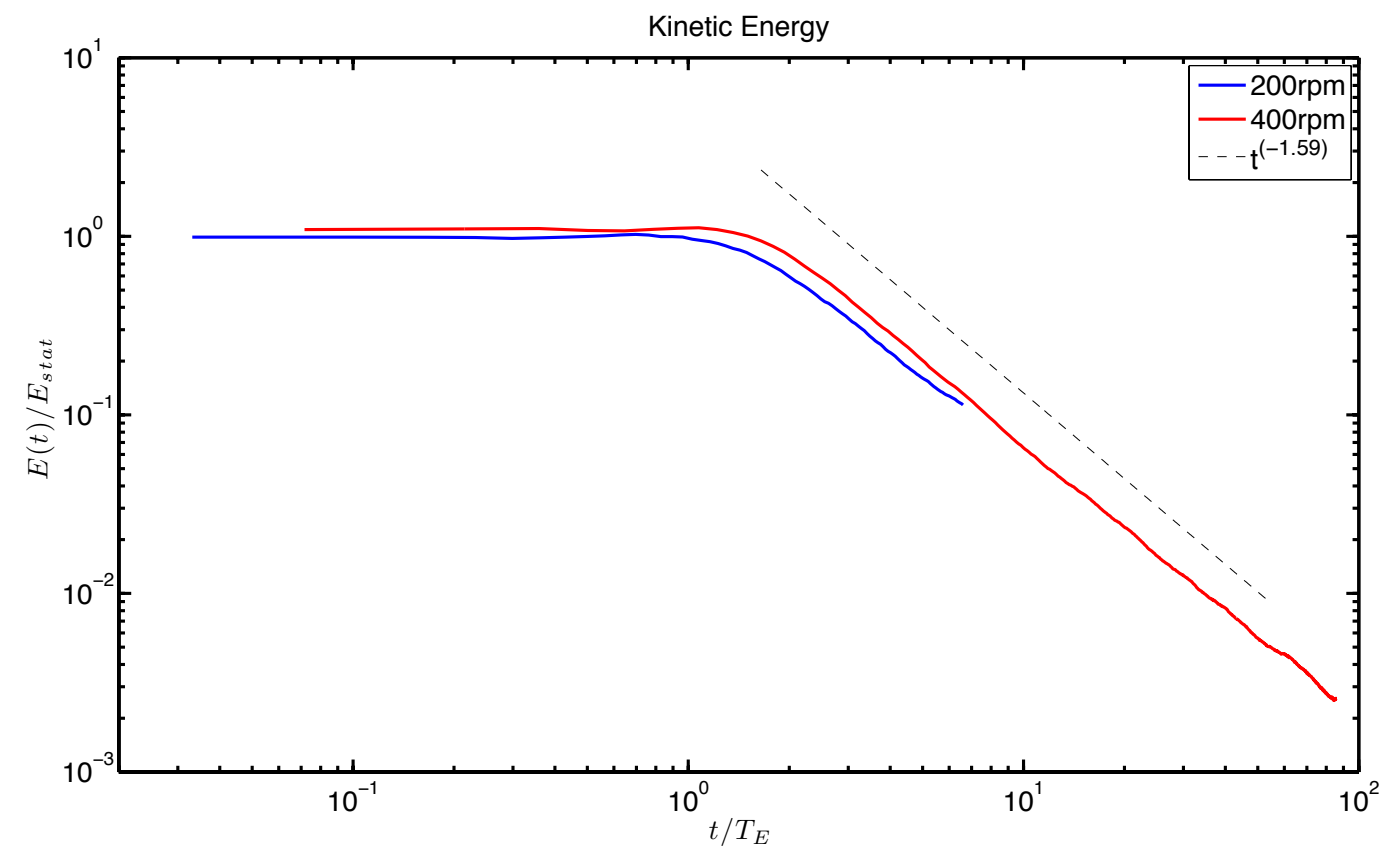

Figure 4.14: Normalized kinetic energy $E(t) / E_{\text {stat }}$ as a function of time, after the motors were switched off. The red curve shows the decay experiment started at the propeller speed of $400 \mathrm{rpm}$ and the blue curve shows the decay experiment started $200 \mathrm{rpm}$. The kinetic energy $E(t)$ is normalized with the kinetic energy of the respective steady-state. Time is normalized with the corresponding large eddy turnover time $T_{E}$. The values for $E_{\text {stat }}$ and $T_{E}$ are listed in Tab. 4.4. The dashed black line corresponds to a $t^{-1.59}$ scaling. 
cases (Fig. 4.6), and the reason is also due to the convection of the more energetic fluid near the propellers to the measurement volume. The decay of the kinetic energy then follows a power law in both cases. From the $400 \mathrm{rpm}$ data, the decay of the kinetic energy after $t \geq 3 T_{E}$ is approximately $t^{-1.59}$. For the $200 \mathrm{rpm}$ case, the data is only plotted up to $6 T_{E}$ because at later times the measurement error in velocities has an appreciable effect on the measured kinetic energy as the fluid velocities decrease with time. Therefore, only the decay from $400 \mathrm{rpm}$ will be further discussed in the following.

In the case of decaying homogeneous turbulence the kinetic energy is related to the energy dissipation rate by

$$
\frac{d E(t)}{d t}=-\epsilon(t)
$$

Since the kinetic energy was measured to decay as $\sim t^{-1.6}$, according to Eq. 4.3, we expect the dissipation rate $\epsilon$ to decay as $\sim t^{-2.6}$. The energy dissipation rate can be directly determined from the inertial range scalings of the structure functions $D_{L L}, D_{N N}$ and $D_{L L L}$ as explained in Section 4.1 and is shown in Fig. 4.15 (the blue curve). For comparison, we also obtained $\epsilon(t)$ from the time derivative of the kinetic energy using Eq. 4.3) and showed the result in the same plot (the green curve), which follows a power-law decay of approximately $t^{-2.6}$ as expected. 
In the period of $3 \leq t / T_{E} \leq 10$, the energy dissipation rate obtained from the structure functions also decays as approximately $t^{-2.6}$ and is, in this range, in good agreement with the energy dissipation rate obtained from the change of the kinetic energy using Eq. 4.3. The departure of the blue curve from the $t^{-2.6}$ scaling at later times is due to the fact that the particle velocities decrease below the level that the measurement error in velocities starts to affect significantly the energy dissipation rate $\epsilon(t)$ determined from the inertial range scalings of the structure functions. On the other hand, the effect of the measurement error on the kinetic energy is relatively small at these times because $E \propto u^{\prime 2}$ while $D_{L L}(r) \propto\left(\delta_{r} u\right)^{2} \propto(r / L)^{2 / 3} u^{\prime 2}$. For the same error in $u^{\prime}$, the relative effect on $D_{L L}(r)$ is thus $(L / r)^{2 / 3}$ times larger, which can be significant for small $r$.

To illustrate that, Fig. 4.16 shows $D_{L L}(r, t)$ as a function of scale at different times. It can be seen that, as time progresses and the measured velocity differences decay, the measurement noise becomes apparent at small $r$, influencing the measurements in the scale range that is used to determine $\epsilon(t)(4-16 \mathrm{~mm}$, marked by the dashed black lines). The plot also shows that the inertial range scaling of $D_{L L}(r, t)$ continuously changes after the energy injection was turned off. For the times later than $10 T_{E}$, the measured $D_{L L}(r, t)$ in Fig. 4.15 changes its slope and the measurement of $\epsilon(t)$ using the scaling $D_{L L}(r, t)=C_{2}(\epsilon r)^{2 / 3}$ becomes unreliable.

In order to investigate the scale-dependent response, Fig. 4.17 shows $D_{L L}(r, t)$, 


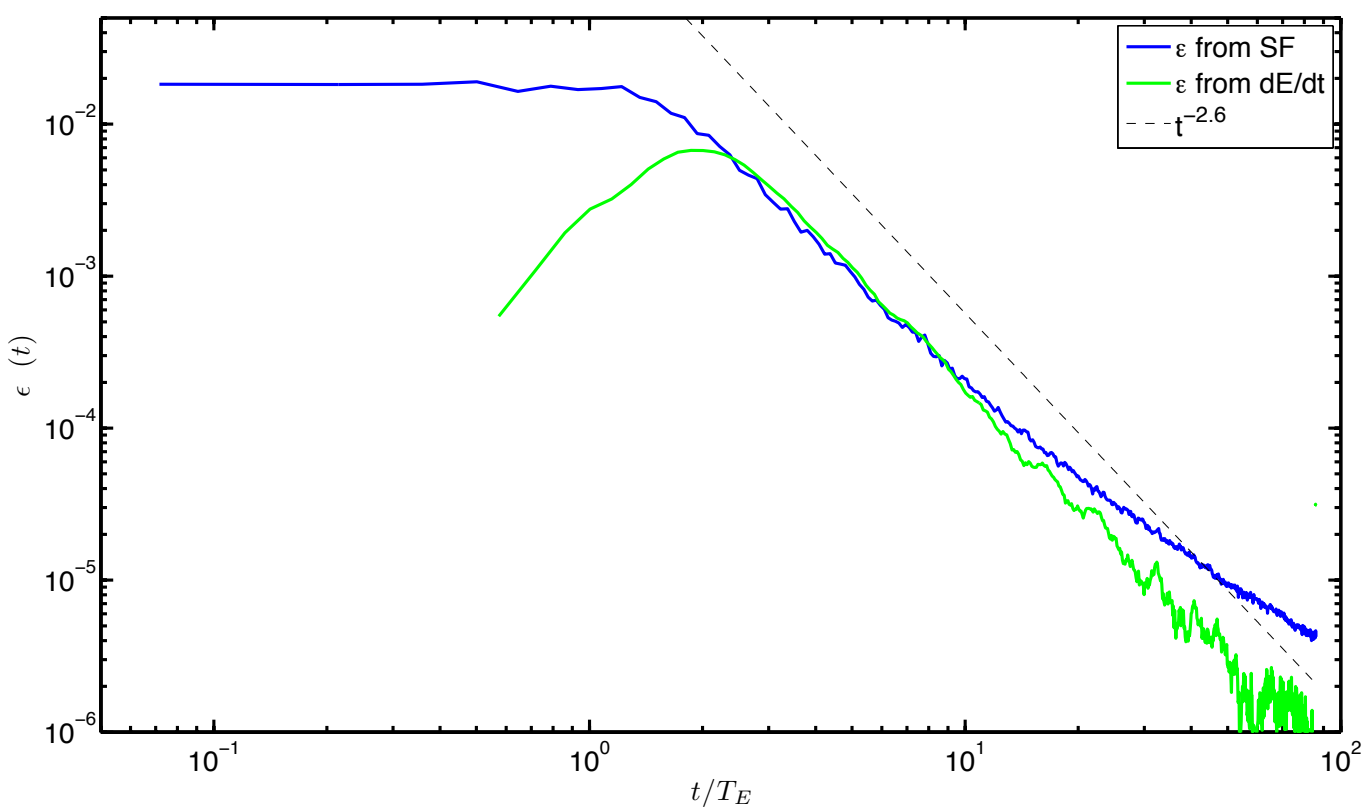

Figure 4.15: Energy dissipation rate as a function of time. The blue curve shows $\epsilon(t)$ obtained from the inertial range scalings of the structure functions $D_{L L}, D_{N N}$ and $D_{L L L}$ according to Eq. (4.2) by averaging over the scale range of $4-16 \mathrm{~mm}$. The green curve shows the energy dissipation rate obtained from the change of the kinetic energy $E(t)$ according to Eq. (4.3). The dashed black line illustrates a $t^{-2.6}$ scaling. 


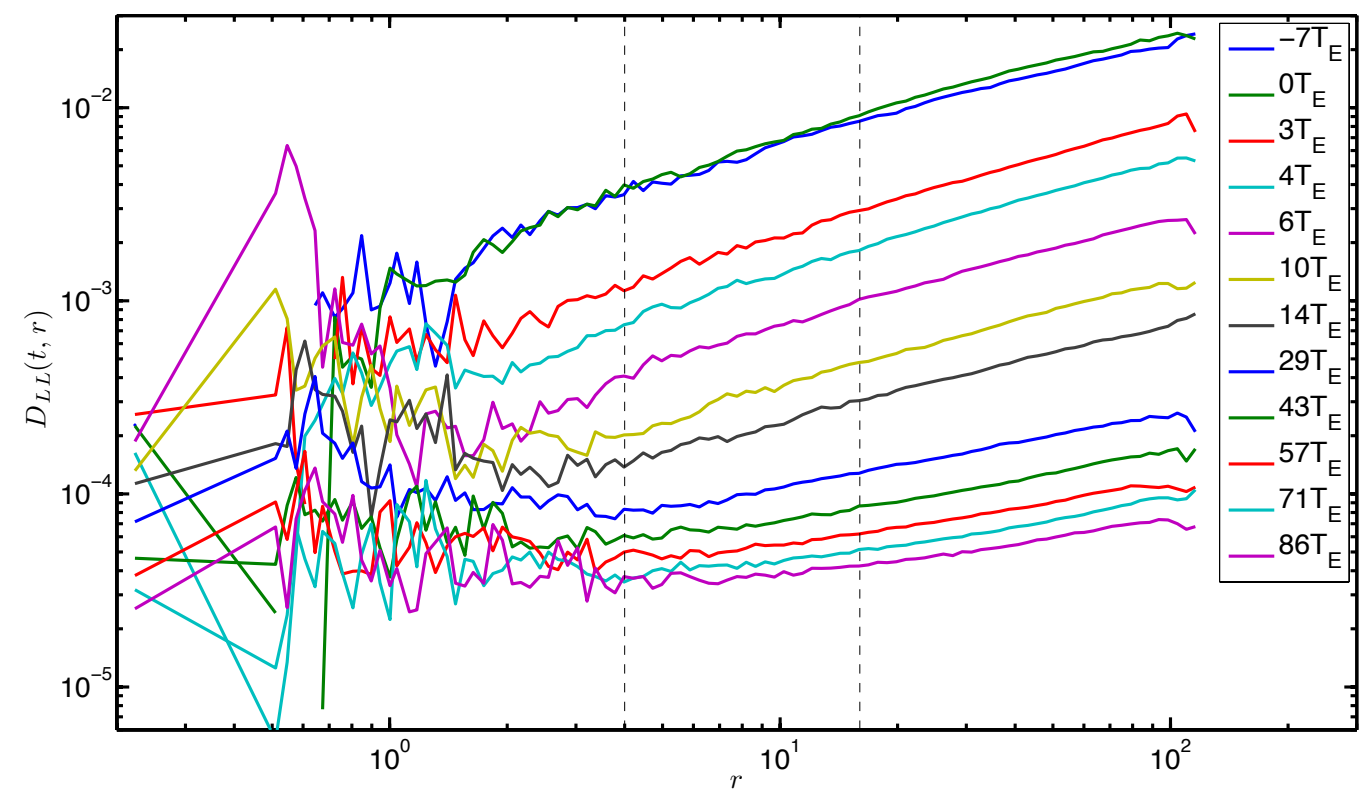

Figure 4.16: $D_{L L}(r, t)$ is shown as a function of $r$, at different times. The black dashed lines indicate the scale range of $4-16 \mathrm{~mm}$ that was used to determine the energy dissipation rate.

normalized by $D_{L L}(r)$ of the steady state, as a function of both scale and time. The color coding and the contour line are chosen to highlight the time when the energy content in each scale drops to $80 \%$ of the energy content of the respective scale at the steady state. Fig. 4.18, Fig. 4.19, and Fig. 4.20 show similar plots highlighting the decay to $50 \%, 20 \%$, and $12 \%$ of the steady state values, respectively. In all cases it can be seen that the small scales decay faster than the large scales. Moreover, 
the effect is more apparent at later times when the decay proceeded.

In stationary turbulence, large scale motion is constantly fed with energy and the small scales dissipate the energy that they receive through the energy cascade. In the case of decaying turbulence, our measurements suggest that after switching off the energy input, the small scales dissipate energy faster than the rate that they receive energy from larger scales. This implies that the inertial range scaling of the second order structure function, known to be $r^{2 / 3}$ in stationary turbulence, slowly changes as the decay of energy progresses. This, together with our observation of the deviation of $D_{L L}(r, t)$ from the $r^{2 / 3}$ scaling in the step-up case (Fig. 4.11, suggest that the scaling laws for non-stationary turbulence can be different from those in stationary turbulence. Our observation therefore has important consequences on theoretical understanding of the decay of turbulence, which is almost exclusively based on the assumption that the inertial range scaling is the same as in the stationary case.

Our finding might be related to recently reported "non-equilibrium" turbulence (Valente \& Vassilicos, 2012; Valente et al., 2014). The exact connection between the two, however, requires further detailed studies. 


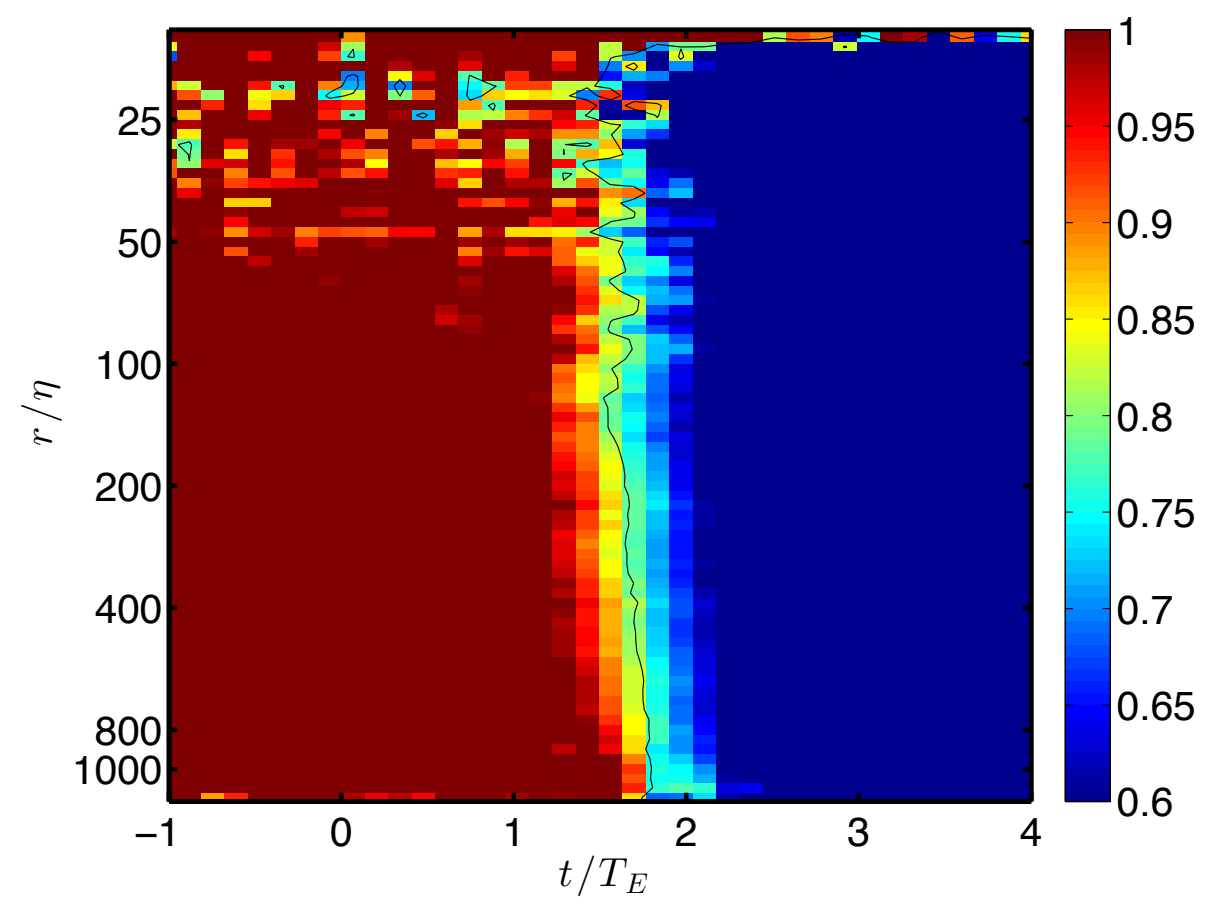

Figure 4.17: $D_{L L}(r, t)$, normalized by $D_{L L}(r)$ of the steady state, as a function of both scale and time. The color coding is chosen to highlight the time when the energy content of each scale drops to $80 \%$ of the energy content of the respective scale at the steady state. The iso-contour corresponds to $D_{L L}(r, t)$ at $80 \%$ of its steady-state value. 


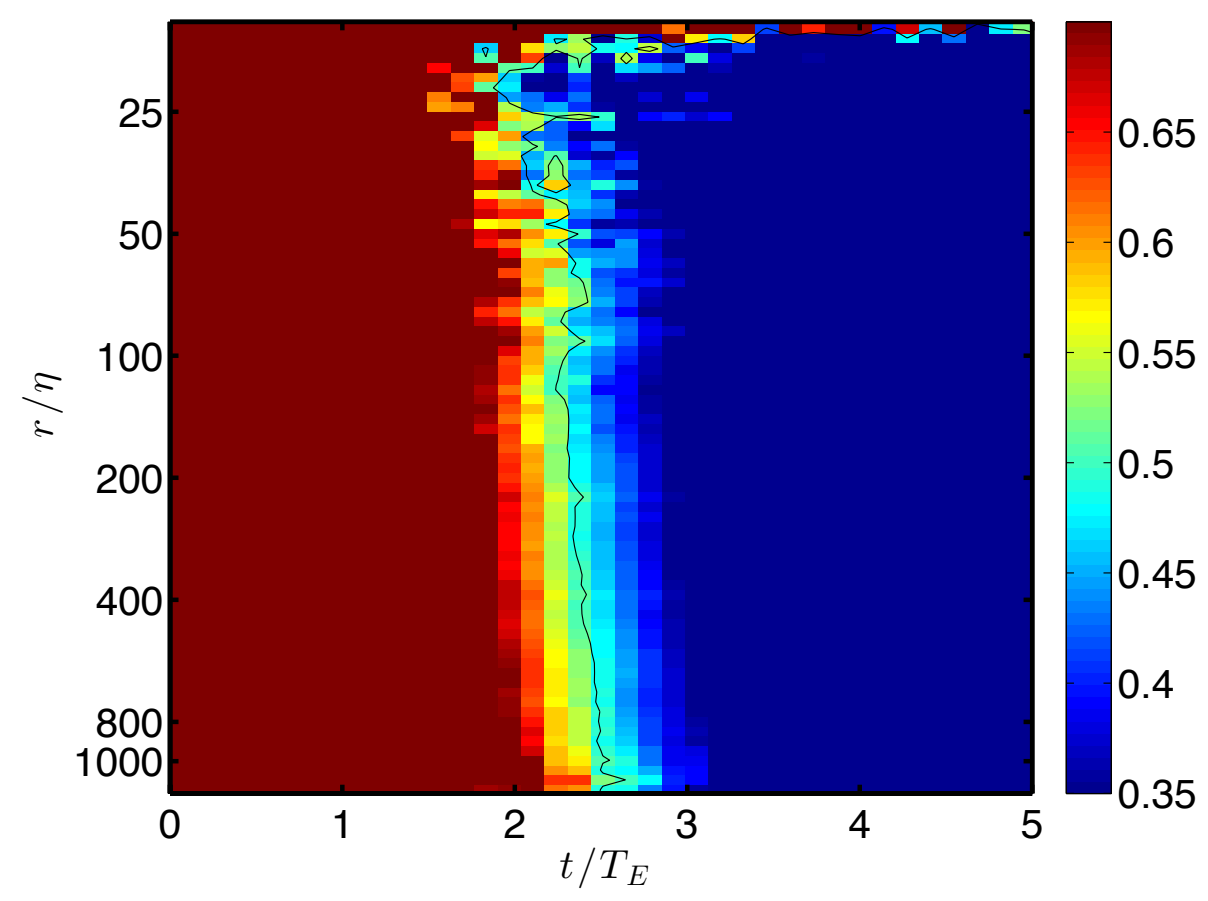

Figure 4.18: $D_{L L}(r, t)$, normalized by $D_{L L}(r)$ of the steady state, as a function of both scale and time. The color coding is chosen to highlight the time when the energy content of each scale drops to $50 \%$ of the energy content of the respective scale at the steady state. The iso-contour corresponds to $D_{L L}(r, t)$ at $50 \%$ of its steady-state value. 


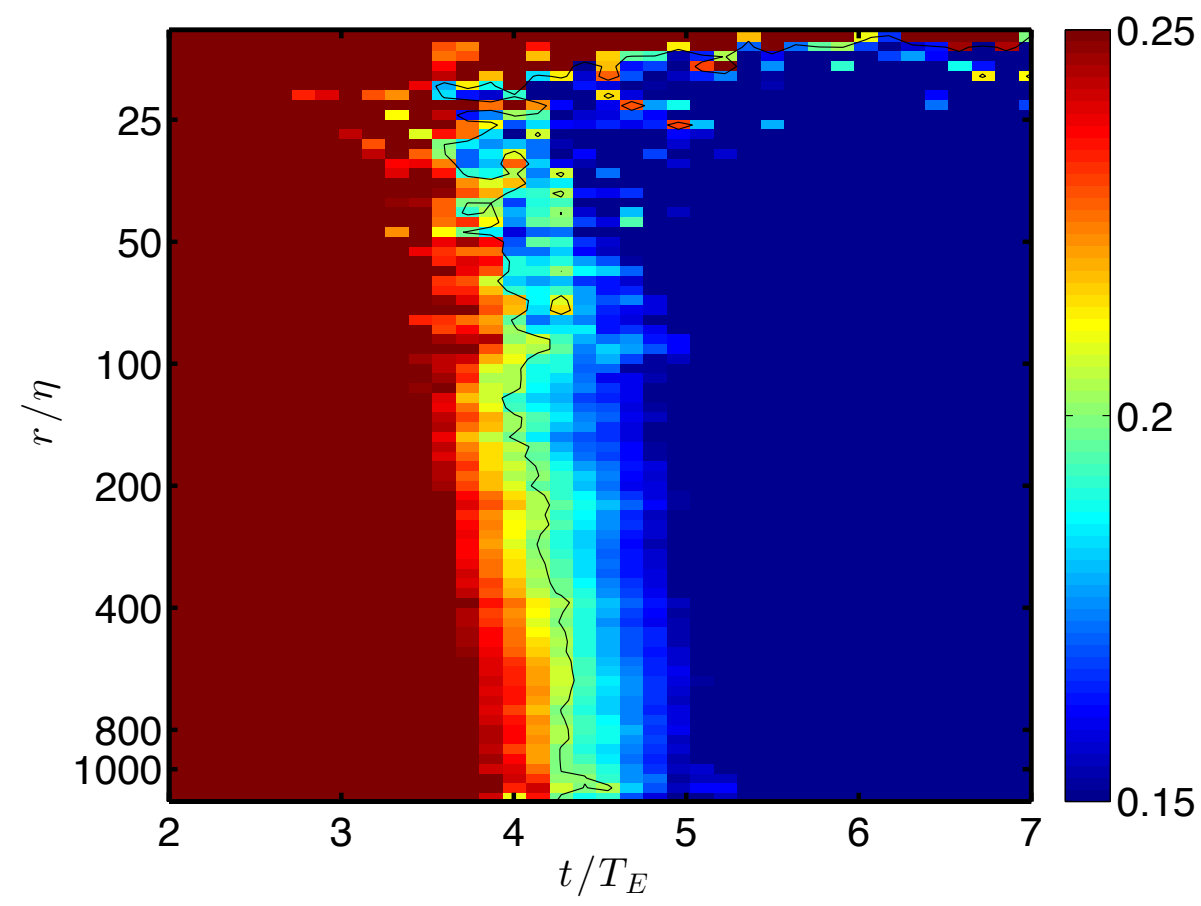

Figure 4.19: $D_{L L}(r, t)$, normalized by $D_{L L}(r)$ of the steady state, as a function of both scale and time. The color coding is chosen to highlight the time when the energy content of each scale drops to $20 \%$ of the energy content of the respective scale at the steady state. The iso-contour corresponds to $D_{L L}(r, t)$ at $20 \%$ of its steady-state value. 


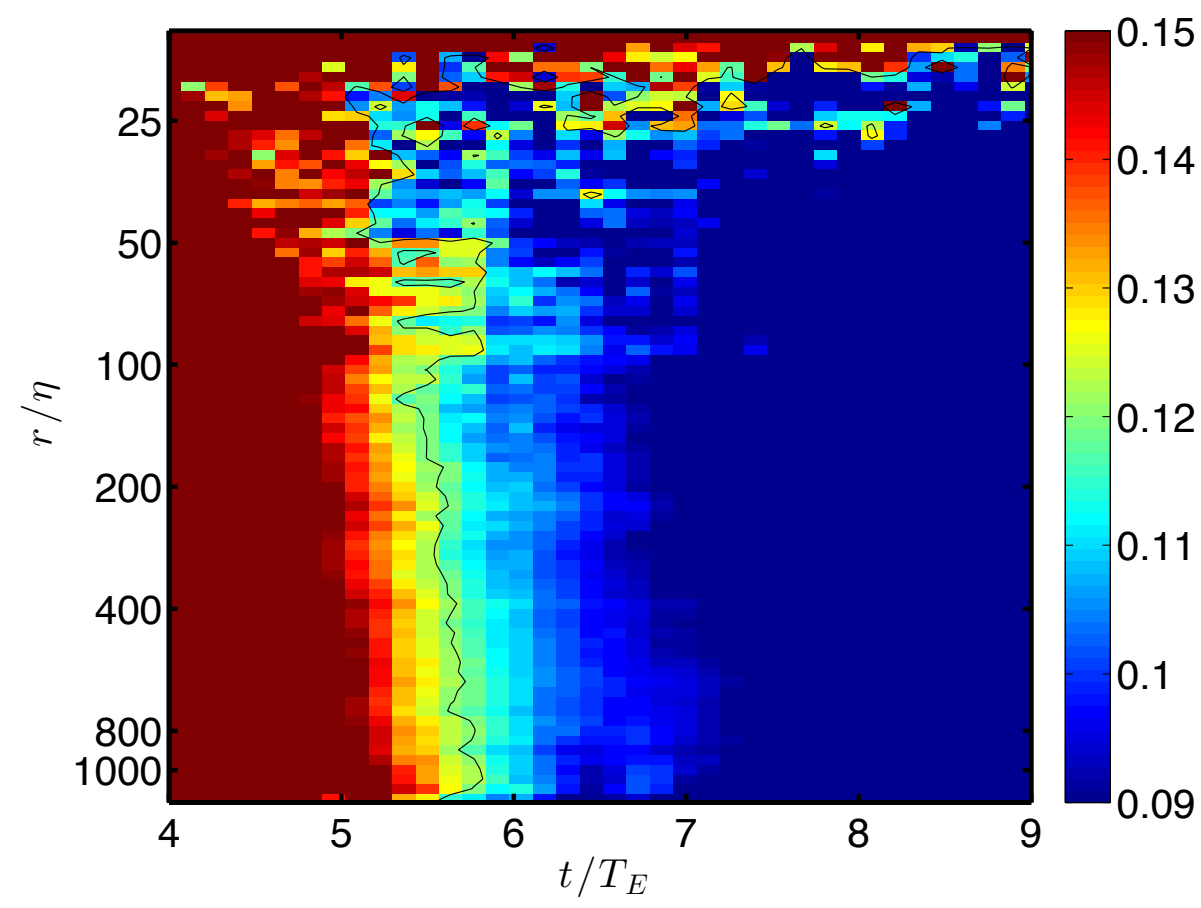

Figure 4.20: $D_{L L}(r, t)$, normalized by $D_{L L}(r)$ of the steady state, as a function of both scale and time. The color coding is chosen to highlight the time when the energy content of each scale drops to $12 \%$ of the energy content of the respective scale at the steady state. The iso-contour corresponds to $D_{L L}(r, t)$ at $12 \%$ of its steady-state value. 


\section{Chapter 5}

\section{Summary and Outlook}

A real-time image compression system was developed, which was demonstrated to reduce the data rate of the high-speed cameras used for Lagrangian Particle Tracking (LPT) by about 90\%. This was achieved by developing an FGPA-based system that pre-evaluated the image data and only wrote data that were significant for the post processing to the hard disc drive. This system extends the capability of our LPT system in two ways: (i) It allows to download high-speed recordings from the internal RAM of the cameras at a data rate of about $1 \mathrm{~GB} / \mathrm{s}$, and therefore reduces the waiting time between two realizations to the order of seconds, rather than the order of minutes when downloading through Ethernet. (ii) In the continuous streaming mode it is possible to gather high-speed recordings with a duration that exceeds the duration of movies recorded to on-board RAM by orders 
of magnitude, as compressed data is written to a hard disc drive whose storage capacity are much larger than the internal RAM.

This system was used to obtain LPT measurements, with a duration of up to one minute, in turbulent flows subject to a sudden change of the large-scale energy input to study the scale-dependent, temporal evolution of these flows. Two different experimental situations were investigated, a step-up increase of the energy input, as well as the decay of turbulence.

In the step-up case it was shown that no significant difference in the response of different scales of turbulence to the change in the forcing could be observed, if the time of the forcing change was used to align the phase averaging of different realizations of the experiment. The reason is that in our experiments, the energy is supplied into the flow through the boundary and the fluctuation in the time needed to connect the injected energy into the observation volume smear out the response. However, when peaks of the kinetic energy signal of the different realizations in the steady part of the measurement were used for alignment of the phase averaging, a clear scale dependence could be measured in the response to the energy peak. As expected, the energy content of the large scales increases before the energy contents of smaller scales pick up. With this signal it was possible to estimate the energy cascade time to be about $0.2-0.3$, large eddy turnover times, in agreement with previous observations from DNS. 
In the case of the decay of turbulence a decay of the kinetic energy with $t^{-1.6}$ was reported and observed for more than ten large-eddy turnover times. The measurement of the decay of the energy dissipation rate was found to be consistent within the measurement uncertainty. An examination of the temporal evolution of the longitudinal second order structure function revealed a faster decay of the small scales compared to the large scales, indicating that the inertial range scaling of the structure function during the decay of turbulence might not be the same as that in stationary turbulence. .

For future investigations, an evaluation of the Lagrangian statistics, as e.g. proposed by Meneveau \& Lund (1994), of the measured data sets will be very interesting and the data should be complemented by measurements with higher spatial and temporal resolution. For better understanding of the phenomena we observe in our experiments, a close collaboration with numerical simulations and theoretical development (e.g. modeling the cascade process) would be essential. Moreover, the apparatus offers a large variety of possibilities concerning the forcing schemes, e.g. periodic forcing or the change from isotropic to anisotropic turbulence, leaving a large number of options to study the response of turbulence to changes in large-scale forcing. .

The developed real-time image compression system will not only find application in experiments like the one described in this thesis, but also in field experi- 
ments on Zugspitze, where LPT measurements in clouds will be performed, and in the planned LPT measurements in the wind tunnel of the Göttingen Turbulence Facility. For this purpose, further development towards the use of an on-cam FPGA instead of an external one is beneficial. 


\section{Bibliography}

Argyris, J., Faust, G., HaAse, M. \& Friedrich, R. 2010 Die Erforschung des Chaos, 2nd edn. Springer.

Avila, K., Moxey, D., de lozar, A., Avila, M., Barkley, D. \& Hof, B. 2011 The onset of turbulence in pipe flow. Science 333, 192-196.

BATChELOR, G. K. 1950 The application of the similarity theory of turbulence to atmospheric diffusion. Q. J. R. Meteorol. Soc. 76, 133-146.

Batchelor, G. K. 1953 The Theory of Homogeneous Turbulence. Cambridge University Press.

Batchelor, G. K. 1967 An Introduction to Fluid Dynamics. Cambridge University Press.

Bodenschatz, E., Bewley, G. P., Nobach, H., Sinhuber, M. \& Xu, H. 2014 Variable density turbulence tunnel facility. Rev. Sci. Instru. 85, 093908. 
Bos, W. J. T., Clark, T. T. \& Rubinstein, R. 2007 Small scale response and modeling of periodically forced turbulence. Phys. Fluids 19, 055107.

Cadot, O., Titon, J. \& Bonn, D. 2003 Experimental observation of resonances in modulated turbulence. J. Fluid Mech. 485, 161-170.

Camussi, R., Ciliberto, S. \& Baudet, C. 1997 Experimental study of the evolution of a velocity perturbation in fully developed turbulence. Phys. Rev. E 56 (5), 6181-6184.

Cekli, H., Tipton, C. \& van De Water, W. 2010 Resonant enhancement of turbulent energy dissipation. Phys. Rev. Lett. 105, 044503.

Chan, K.-Y., Stich, D. \& Voth, G. A. 2007 Real-time image compression for high-speed particle tracking. Rev. Sci. Instru. 78, 023704.

Chien, C.-C., Blum, D. B. \& Voth, G. A. 2013 Effects of fluctuating energy input on the small scales in turbulence. J. Fluid Mech. 737, 527-551.

Comte-Bellot, G. \& Corrsin, S. 1971 Simple Eulerian time correlation of full- and narrow-band velocity signals in grid-generated, 'isotropic' turbulence. J. Fluid Mech. 48, 273-337.

Davidson, P. A. 2004 Turbulence: An Introduction for Scientists and Engineers. Oxford University Press. 
Di LoRenzo, F. 2010 Development of a receiver system for the RTO. Master's thesis, University of Applied Sciences, Saarbrücken.

Díaz, J., Ros, E., Pelayo, F., Ortigosa, E. M. \& Mota, S. 2006 FPGAbased real-time optical-flow system. IEEE Trans. Circuits Syst. for Video Technol. 16 (2), 274-279.

Dracos, T. 1996 Particle tracking in three-dimensional space. In ThreeDimensional Velocity and Vorticity Measuring and Image Analysis Techniques (ed. T. Dracos), pp. 129-152. Kluwer Academic Publishers.

Frisch, U. 1995 Turbulence: The Legacy of A. N. Kolmogorov. Cambridge University Press.

George, W. K. 1992 The decay of homogeneous isotropic turbulence. Phys. Fluids A 4, 1492-1509.

Gerrard, J. H. 1971 An experimental investigation of pulsating turbulent water flow in a tube. J. Fluid Mech. 46, 43-64.

Hamlington, P. E. \& Dahm, W. J. A. 2009 Frequency response of periodically sheared hohogeneous turbulence. Phys. Fluids 21, 055107.

He, S. \& JACKson, J. D. 2009 An experimental study of pulsating turbulent flow in a pipe. Eur. J. Mech. B/Fluids 28, 309-320. 
He, S. \& Seddighi, M. 2013 Turbulence in transient channel flow. J. Fluid Mech. 715, 60-102.

Hinze, J. 1975 Turbulence, 2nd edn. McGraw-Hill.

Hooghoudt, J.-O., Lohse, D. \& Toschi, F. 2001 Decaying and kicked turbulence in a shell model. Phys. Fluids 13 (7), 2013-2018.

Ishida, T., Davidson, P. A. \& KanedA, Y. 2006 On the decay of isotropic turbulence. J. Fluid Mech. 564, 455-475.

Jin, S., Cho, J., Pham, X. D., Lee, K. M., Park, S.-K., Kim, M. \& JEON, J. W. 2010 FPGA design and implementation of a real-time stereo vision system. IEEE Trans. Circuits Syst. for Video Technol. 20 (1), 15-26.

JiN, X.-L. \& XIA, K.-Q. 2008 An experimental study of kicked thermal turbulence. J. Fluid Mech. 606, 133-151.

Kolmogorov, A. N. 1941a Dissipation of energy in locally isotorpic turbulence. Dokl. Akad. Nauk. SSSR 32, 16-18.

Kolmogorov, A. N. $1941 b$ The local structure of turbulence in incompressible viscous fluid for very large reynolds numbers. Dokl. Akad. Nauk SSSR 30, 301305. 
Kreizer, M. \& Liberzon, A. 2011 Three-dimensional particle tracking method using FPGA-based real-time image processing and four-view image splitter. Exp. Fluids 50, 613-620.

Kreizer, M., Ratner, D. \& Liberzon, A. 2010 Real-time image processing for particle tracking velocimetry. Exp. Fluids 48, 105-110.

Krogstad, P.-A. \& Davidson, P. A. 2010 Is grid turbulence Saffman turbulence? J. Fluid Mech. 642, 373-394.

Kuczaj, A. K., Geurts, B. J. \& Lohse, D. 2006 Response maxima in timemodulated turbulence: Direct numerical simulations. Europhys. Lett. 73 (6), $851-857$.

Kuczaj, A. K., Geurts, B. J., Lohse, D. \& van de Water, W. 2008 Turbulence modification by periodically modulated scale-dependent forcing. Comput. Fluids 37, 816-824.

Labbé, R., Baudet, C. \& Bustamante, G. 2007 Experimental evidence of accelerated energy transfer in turbulence. Phys. Rev. E 75, 016308.

Lavoie, P., Djenidi, L. \& Antonia, R. A. 2007 Effects of initial conditions in decaying turbulence generated by passive grids. J. Fluid Mech. 585, 395-420.

Lohse, D. 2000 Periodically kicked turbulence. Phys. Rev. E 62 (4), 4946-4949. 
Mann, J., Otт, S. \& Andersen, J. S. 1999 Experimental study of relative, turbulent diffusion. Tech. Rep. Risø-R-1036(EN). Risø National Laboratory.

Meneveau, C. \& Lund, T. S. 1994 On the Lagrangian nature of the turbulence energy cascade. Phys. Fluids 6, 2820-2825.

Monin, A. S. \& Yaglom, A. M. 1975 Statistical Fluid Mechanics, , vol. 2. MIT Press.

Nolting, W. 2007 Grundkurs Theoretische Physik 3, 8th edn. Springer Lehrbuch.

Ouellette, N. T., Xu, H. \& Bodenschatz, E. $2006 a$ A quantitative study of three-dimensional Lagrangian particle tracking algorithms. Exp. Fluids 40, $301-313$.

Ouellette, N. T., Xu, H., Bourgoin, M. \& Bodenschatz, E. $2006 b$ An experimental study of turbulent relative dispersion models. New J. Phys. 8 (109).

Pearson, B. R., Yousef, T. A., Haugen, N. E. L., Brandenburg, A. \& Krogstad, P.-A. 2004 Delayed correlation between turbulent energy injection and dissipation. Phys. Rev. E 70, 056301.

Pope, S. 2000 Turbulent Flows. Cambridge University Press.

Pumir, A. 1996 Turbulence in homogeneous shear flows. Phys. Fluids 8 (11), $3112-3127$. 
RAmaprian, B. R. \& Tu, S. W. 1983 Fully developed periodic turbulent pipe flow. Part 2. The detailed structure of the flow. J. Fluid Mech. 137, 59-81.

REYNOLDS, O. 1883 An experimental investigation of the circumstances which determine whether the motion of water shall be direct or sinuous, and of the law of resistance in parallel channels. Philos. Trans. R. Soc. London 174, 935-982.

Richardson, L. F. 1922 Weather Prediction by Numerical Process. Cambridge University Press.

Risius, S. 2012 Investigation of turbulent flows at the umweltforschungsstation schneefernerhaus (zugspitze). Diploma Thesis, University of Göttingen.

Risius, S., Xu, H., Di Lorenzo, F., Xi, H.-D., Siebert, H., Shaw, R. A. \& Bodenschatz, E. 2015 Schneefernerhaus as a mountain research station for clouds and turbulence. Part 1: Flow conditions and large-scale turbulence. Atmos. Meas. Tech. Discuss. 8, 541-568.

Saddoughi, S. G. \& Veeravalli, S. V. 1994 Local isotropy in turbulent boundary layers at high-Reynolds number. J. Fluid Mech. 268, 333-372.

Saffman, P. G. $1967 a$ The large-scale structure of homogeneous turbulence. J. Fluid Mech. 27, 581-593. 
Saffman, P. G. $1967 b$ Note on decay of homogeneous turbulence. Phys. Fluids 10, 1349.

Siebert, H., Shaw, R. A., Ditas, J., Schmeissner, T., Malinowski, S. P., Bodenschatz, E. \& Xu, H. 2015 Schneefernerhaus as a mountain research station for clouds and turbulence. Part 2: Cloud microphysics and fine-scale turbulence. Atmos. Meas. Tech. Discuss. 8, 569-597.

Sinhuber, M., Bodenschatz, E. \& Bewley, G. P. 2015 On the degeneration of turbulence at high Reynolds numbers. Phys. Rev. Lett. 114, 034501.

Snyder, W. H. \& Lumley, J. L. 1971 Some measurements of particle velocity autocorrelation functions in a turbulent flow. J. Fluid Mech. 48, 41-71.

Sreenivasan, K. R. 1995 On the universality of the Kolmogorov constant. Phys. Fluids 7, 2778-2784.

Stalp, S. R., Skrbek, L. \& Donnelly, R. J. 1999 Decay of grid turbulence in a finite channel. Phys. Rev. Lett. 82, 4831-4834.

Taylor, G. I. 1935 Statistical theory of turbulence. Proc. Roy. Soc. Lond. A 151, 421-444.

Teitelbaum, T. \& Mininni, P. D. 2009 Effect of helicity and rotation on the free decay of turbulent flows. Phys. Rev. Lett. 103. 
Teitelbaum, T. \& Mininni, P. D. 2011 The decay of turbulence in rotating flows. Phys. Fluids 23, 065105.

Toschi, F. \& Bodenschatz, E. 2009 Lagrangian properties of particles in turbulence. Annu. Rev. Fluid Mech. 41, 375-404.

TsAI, R. Y. 1987 A versatile camera calibration technique for high-accuracy 3D machine vision metrology using off-the-shelf tv cameras and lenses. IEEE J. Robot. Autom. RA-3 (4), 323-344.

Tu, S. W. \& Ramaprian, B. R. 1983 Fully developed periodic turbulent pipe flow. Part 1. Main experimental results and comparison with predictions. J. Fluid Mech. 137, 31-58.

Valente, P. C., Onishi, R. \& DA Silva, C. B. 2014 Origin of the imbalance between energy cascade and dissipation in turbulence. Phys. Rev. E 90, 023003.

Valente, P. C. \& Vassilicos, J. C. 2012 Universal dissipation scaling for nonequilibrium turbulence. Phys. Rev. Lett. 108, 214503.

von der Heydt, A., Grossmann, S. \& Lohse, D. $2003 a$ Response maxima in modulated turbulence. Phys. Rev. E 67, 046308.

von der Heydt, A., Grossmann, S. \& Lohse, D. $2003 b$ Response maxima in modulated turbulence. ii. numerical simulations. Phys. Rev. E 68, 066302. 
von Kármán, T. \& Howarth, L. 1938 On the statistical theory of isotropic turbulence. Proc. R. Soc. London Ser. A 164 (917), 192-215.

XU, H. 2008 Tracking Lagrangian trajectories in position-velocity space. Meas. Sci. Technol. 19, 075105.

Yeung, P. K. 2002 Lagrangian investigations of turbulence. Annu. Rev. Fluid Mech. 34, 115-142.

Zimmermann, R. 2008 The Lagrangian Exploration Module. Diploma Thesis, University of Göttingen.

Zimmermann, R., Xu, H., Gasteuil, Y., Bourgoin, M., Volk, R., PinTOn, J.-F. \& Bodenschatz, E. 2010 The Lagrangian exploration module: An apparatus for the study of statistically homogeneous and isotropic turbulence. Rev. Sci. Instru. 81, 055112. 


\section{Acknowledgments}

First and foremost I want to thank my supervisor Eberhard Bodenschatz for giving me the great opportunity to conduct my doctoral research in his group. None of this would have been possible without his support at any time through the years.

I am very grateful for Haitao Xu's support. I learned so much in our countless discussions and I was very happy to have him as a source of virtually endless knowledge about turbulence and experimental procedures. I also thank him for not getting tired, even if I asked the same question twice.

I also want to thank Jörg Enderlein and Björn Hof for their support as part of my Thesis Committee.

Furthermore, I want to thank our technical staff at the MPIDS: Achim, Andreas K., Andreas R., Arthur, Gerhard, Marcel, Ortwin, Thomas, Zvonko, and the whole work shop team around Udo Schminke for providing and maintaining everything that comes out or goes into the wall and for building many needed bits and pieces. 
I want to thank Radu Corlan from Vision Research for his support and countless emails to teach me the communication protocol of the cameras.

A very big thank-you to goes to Mathieu Gibert for his help in debugging FPGA codes in the very early stages of this endeavor, for reading the matrix with me and becoming a great friend.

Thank you, to all the friends I made in the nearly six years I spent in Göttingen. Thanks for all the fun we had, for good days at work, for good days off work, for cheering me up, for motivating me, for distracting me.

Last but not least I want to thank my family. It was your endless support that helped me through this. Thank you for giving me advice, motivation and an outside perspective. 


\section{Curriculum Vitae}

\section{Personal Data}

$\begin{array}{ll}\text { Name } & \text { Fabio Di Lorenzo } \\ \text { Date of birth } & \text { May 11, 1982 } \\ \text { Nationality } & \text { Italian }\end{array}$

\section{Education}

$\begin{array}{ll}\text { 05/2010 - today } & \text { PhD studies in Physics } \\ & \text { MPI for Dynamics and Self-Organisation, Göttingen, Germany } \\ & \text { International Max Planck Research School for Physics of Biologi- } \\ \text { cal and Complex Systems (IMPRS PBCS) } & \text { Master studies in Mechatronics/Sensortechnology } \\ \text { 04/2008-05/2010 } & \text { University of Applied Sciences, Saarbrücken, Germany } \\ & \text { Thesis title: } \\ & \text { "Development of a receiver system for the RTO" } \\ & \text { conducted at the MPI for Dynamics and Self-Organisation, Göt- } \\ & \text { tingen, Germany } \\ & \text { Bachelor studies in Mechatronics/Sensortechnology } \\ & \text { University of Applied Sciences (HTW), Saarbrücken, Germany } \\ & \text { Thesis title: } \\ \text { "Aufbau einer LDA-Optik und Inbetriebnahme eines MIOC" } & \text { conducted in the Laboratory for optical measurement technology } \\ \text { at the HTW } & \begin{array}{l}\text { Apprenticeship in Mechatronics } \\ \text { Deutsche Steinkohle AG, Völklingen, Germany }\end{array} \\ & \begin{array}{l}\text { German university entrance qualification } \\ \text { Technisches Gymnasium, Völklingen, Germany }\end{array}\end{array}$




\section{Teaching Experience}

10/2011-09/2012 Teaching assistant for the undergraduate courses in Physics I (Mechanics) and Physics II (Electrodynamics) at the Georg August University, Göttingen, Germany

04/2007-04/2009 Teaching assistant for the undergraduate courses in Electronic Engineering, Electrical Engineering and Sensortechnology at the University of Applied Sciences, Saarbrücken, Germany

09/2010 Teaching assistant for a Math preparatory course at the University of Applied Sciences, Saarbrücken, Germany

Talks, Posters and Conferences

09/2013 $14^{\text {th }}$ European Turbulence Conference 2013

"Experimental Study of Isotropic Turbulence under Time-Dependent Forcing"

Lyon, France

09/2011 $13^{\text {th }}$ European Turbulence Conference 2011

"Real-Time image compression on Lagrangian Particle Tracking data"

Warsaw, Poland

08/2011 Co-Organizer of the 5th European Post-Gradued Fluid Dynamics Conference

Göttingen, Germany

Göttingen, December 12, 2014 